\title{
Integrative approach to interpret DYRK1A variants, leading to a frequent neurodevelopmental disorder
}

Jeremie Courraud ${ }^{1,2,3,4}$, Eric Chater-Diehl ${ }^{5}$, Benjamin Durand ${ }^{1,2,3,4}$, Marie Vincent ${ }^{6}$, Maria del Mar Muniz Moreno $^{1,2,3,4}$, Imène Boujelbene ${ }^{1,2,3,4,7}$, Nathalie Drouot ${ }^{1,2,3,4}$, Loréline Genschik ${ }^{1,2,3,4}$, Elise Schaefer ${ }^{8}$, Mathilde Nizon ${ }^{6}$, Bénédicte Gerard ${ }^{7}$, Marc Abramowicz ${ }^{9}$, Benjamin Cogné ${ }^{6}$, Lucas Bronicki ${ }^{10}$, Lydie Burglen $^{11}$, Magalie Barth ${ }^{12}$, Perrine Charles ${ }^{13}$, Estelle Colin ${ }^{12}$, Christine Coubes ${ }^{14}$, Albert David ${ }^{6}$, Bruno Delobel $^{15}$, Florence Demurger ${ }^{16}$, Sandrine Passemard ${ }^{17}$, Anne-Sophie Denommé ${ }^{18}$, Laurence Faivre ${ }^{18}$, Claire Feger $^{7}$, Mélanie Fradin ${ }^{20}$, Christine Francannet ${ }^{21}$, David Genevieve ${ }^{14}$, Alice Goldenberg ${ }^{22}$, Anne-Marie Guerrot $^{22}$, Bertrand Isidor ${ }^{6}$, Katrine M. Johannesen ${ }^{24,25}$, Boris Keren ${ }^{13}$, Maria Kibæk ${ }^{23}$, Paul Kuentz ${ }^{18}$, Michele Mathieu-Dramard ${ }^{26}$, Bénédicte Demeer ${ }^{26}$, Julia Metreau ${ }^{27}$, Rikke Steensbjerre Møller ${ }^{24,25}$, Sébastien Moutton $^{18}$, Laurent Pasquier ${ }^{20}$, Kristina Pilekær Sørensen ${ }^{23}$, Laurence Perrin ${ }^{28}$, Mathilde Renaud ${ }^{29}$, Pascale Saugier $^{22}$, Marlene Rio ${ }^{30}$, Joane Svane ${ }^{23}$, Julien Thevenon ${ }^{31}$, Frederic Tran Mau Them ${ }^{18}$, Cathrine Elisabeth Tronhjem $^{23}$, Antonio Vitobello ${ }^{18}$, Valerie Layet $^{32}$, Stéphane Auvin ${ }^{33}$, Khaoula Khachnaoui ${ }^{34}$, MarieChristine Birling $^{35}$, Severine Drunat ${ }^{36}$, Allan Bayat ${ }^{23}$, Christèle Dubourg ${ }^{37}$, Salima El Chehadeh ${ }^{7}$, Christina Fagerberg $^{23}$, Cyril Mignot ${ }^{12}$, Michel Guipponi ${ }^{9}$, Thierry Bienvenu ${ }^{38}$, Yann Herault ${ }^{1,2,3,4}$, Julie Thompson ${ }^{39}$, Marjolaine Willems ${ }^{14}$, Jean-Louis Mandel ${ }^{1,2,3,4}$, Rosanna Weksberg ${ }^{5,40,41,42,43}$, *Amélie Piton 1,2,3,4,7,44

\section{AFFILIATIONS}

${ }^{1}$ Institut de Génétique et de Biologie Moléculaire et Cellulaire, Illkirch 67400, France

${ }^{2}$ Centre National de la Recherche Scientifique, UMR7104, Illkirch 67400, France

${ }^{3}$ Institut National de la Santé et de la Recherche Médicale, U964, Illkirch 67400, France

${ }^{4}$ Université de Strasbourg, Illkirch 67400, France

${ }^{5}$ Genetics and Genome Biology, The Hospital for Sick Children, Toronto, ON M5G 1X8, Canada

${ }^{6}$ Service de Génétique Médicale, CHU de Nantes \& Inserm, CNRS, Université de Nantes, l’institut du thorax, 44000 Nantes,

France

${ }^{7}$ Unité de Génétique Moléculaire, IGMA, Hôpitaux Universitaire de Strasbourg, Strasbourg, France

${ }^{8}$ Service de Génétique Médicale, IGMA, Hôpitaux Universitaires de Strasbourg, Strasbourg, France

${ }^{9}$ Service of Genetic Medicine, University Hospitals of Geneva, Geneva, Switzerland

${ }^{10}$ Department of Genetics, CHEO, Ottawa, ON, Canada.

${ }^{11}$ Centre de référence des malformations et maladies congénitales du cervelet et Département de génétique et embryologie médicale, APHP, Sorbonne Université, Hôpital Armand Trousseau, 75012 Paris, France

${ }^{12}$ Pediatrics \& Biochemistry and Genetics, Department, Angers Hospital, Angers, France.

${ }^{13}$ Genetic Department, University Hospital Pitié-Salpêtrière, AP-HP, Paris, France

${ }^{14}$ Département de Génétique Médicale maladies rares et médecine personnalisée, Centre de Référence Maladies Rares Anomalies du Développement, Hôpital Arnaud de Villeneuve, Université Montpellier, France

${ }^{15}$ Centre de Génétique Chromosomique, GHICL, Hôpital Saint Vincent de Paul, Lille, France

${ }^{16}$ Service de Génétique, CH Bretagne Atlantique- Vannes

${ }^{17}$ Département de Génétique, Hôpital Universitaire Robert Debré, APHP, Paris, France.

${ }^{18}$ Centre de Génétique et Centre de Référence Anomalies du développement et Syndromes malformatifs, Hôpital d’Enfants and INSERM UMR1231 GAD, FHU TRANSLAD, CHU de Dijon, Dijon, France

${ }^{19}$ Unité Fonctionnelle d’Innovation en Diagnostique Génomique des Maladies Rares, Pôle de Biologie, FHU-TRANSLAD, CHU Dijon Bourgogne, F-21000, Dijon, France

${ }^{20}$ Centre de Référence Maladies Rares, Unité Fonctionnelle de Génétique Médicale, CHU, Rennes, France

${ }^{21}$ Service de Génétique médicale, CHU de Clermont-Ferrand, Clermont-Ferrand, France

${ }^{22}$ Normandie Univ, UNIROUEN, Inserm U1245 and Rouen University Hospital, Department of Genetics and Reference Center for Developmental Disorders , F 76000, Normandy Center for Genomic and Personalized Medicine, Rouen, France

${ }^{23}$ Department of Clinical Genetics, Odense Denmark Hospital, Odense University Hospital, Odense, Denmark

${ }^{24}$ Department of Epilepsy Genetics and Personalized Treatment, The Danish Epilepsy Centre, Dianalund, Denmark

${ }^{25}$ Institute for Regional Health Services, University of Southern Denmark, Odense Denmark

${ }^{26}$ Service de Génétique Clinique, Centre de référence maladies rares, CHU d'Amiens-site Sud, Amiens, France

${ }^{27}$ APHP, Service de neurologie pédiatrique, Hôpital Universitaire Bicetre, Le Kremlin-Bicetre, France

${ }^{28}$ Department of Genetics, Robert Debré Hospital, AP-HP, Paris, France

${ }^{29}$ Service de Génétique Clinique et de Neurologie, Hôpital Brabois Enfants, Nancy, France

${ }^{30}$ Department of medical genetics and reference centre for rare intellectual disabilities. INSERM UMR 1163, Paris Descartes-

Sorbonne Paris Cité University, Imagine Institute, Necker Enfants Malades Hospital, Paris, France 
${ }^{31}$ Department of Genetics and Reproduction, Centre Hospitalo-Universitaire Grenoble-Alpes, Grenoble, France

${ }^{32}$ Consultations de génétique, Groupe Hospitalier du Havre, Le Havre, France

${ }^{33}$ Center for rare epilepsies \& epilepsy unit Robert-Debré Hospital, APHP, \& INSERM NeuroDiderot, Université de Paris,

Paris, France

${ }^{34}$ Université Côte d’Azur, Inserm U1081, CNRS UMR7284, IRCAN, CHU de Nice, Nice, France

${ }^{35}$ ICS, Mouse Clinical Institute, Illkirch-Graffenstaden, France

${ }^{36}$ Département de Génétique, Hôpital Universitaire Robert Debré, Paris

${ }^{37}$ Laboratoire de Génétique Moléculaire, CHU Pontchaillou, UMR 6290 CNRS, IGDR, Faculté de Médecine, Université de Rennes 1, Rennes, France

${ }^{38}$ Molecular Genetics Laboratory, Cochin Hospital, APHP.Centre-Université de Paris, and INSERM UMR 1266, Institut de Psychiatrie et de Neurosciences de Paris, 75014 Paris, France

${ }^{39}$ Complex Systems and Translational Bioinformatics (CSTB), ICube laboratory - CNRS, Fédération de Médecine

Translationnelle de Strasbourg (FMTS), University of Strasbourg, Strasbourg, France

${ }^{40}$ Division of Clinical and Metabolic Genetics, The Hospital for Sick Children, Toronto, Ontario, M5G 1X8, Canada

${ }^{41}$ Department of Molecular Genetics, University of Toronto, Toronto, Ontario, M5S 1A1, Canada-RBW

${ }^{42}$ Department of Pediatrics, University of Toronto, Toronto, ON, Canada

${ }^{43}$ Institute of Medical Science, School of Graduate Studies, University of Toronto, Toronto, Ontario, Canada

${ }^{44}$ Institut Universitaire de France

The authors declare no conflict of interest.

*Address for correspondence and material request:

Amélie Piton, $\mathrm{PhD}$

Laboratoire "Mécanismes génétiques des maladies neurodéveloppementales", IGBMC, Illkirch, France

Tel : +33369551652

E-mail: piton@igbmc.fr

\section{Competing interests}

None.

\section{ABSTRACT}

PURPOSE: DYRK1A syndrome is among the most frequent monogenic forms of intellectual disability (ID). We refined the molecular and clinical description of this disorder and developed tools to improve interpretation of missense variants, which remains a major challenge in human genetics.

METHODS: We reported clinical and molecular data for fifty individuals with ID harboring DYRK1A variants and developed i) a specific DYRK1A clinical score, ii) amino acid conservation data generated from one hundred of DYRK1A sequences across different taxa, iii) in vitro overexpression assays to study level, cellular localization, and kinase activity of DYRK1A mutant proteins, and iv) a specific blood DNA methylation signature.

RESULTS: This integrative approach was successful to reclassify several variants as pathogenic. However, we questioned the involvement of some others, such as p.Thr588Asn, still reported as likely pathogenic, and showed it does not cause an obvious phenotype in mice.

CONCLUSION: Our study demonstrated the need for caution when interpreting variants in DYRK1A, even those occurring de novo. The tools developed will be useful to interpret accurately the variants identified in the future in this gene. 


\section{Integrative tools to interpret variants causing DYRK1A syndrome}

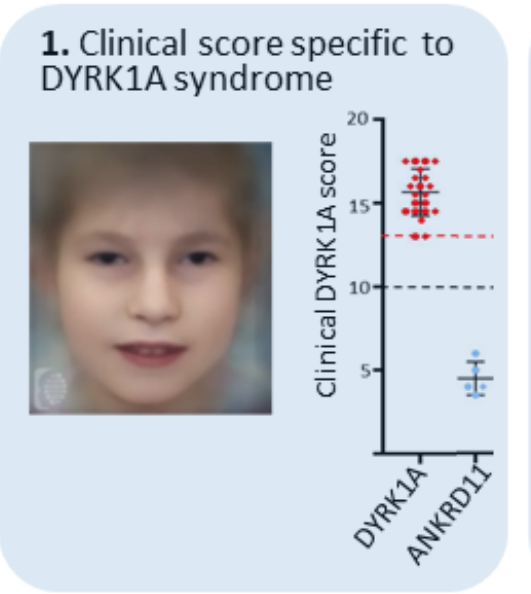

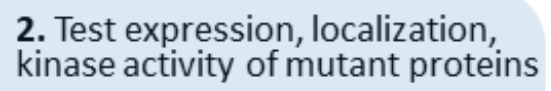
kinase activity of mutant proteins

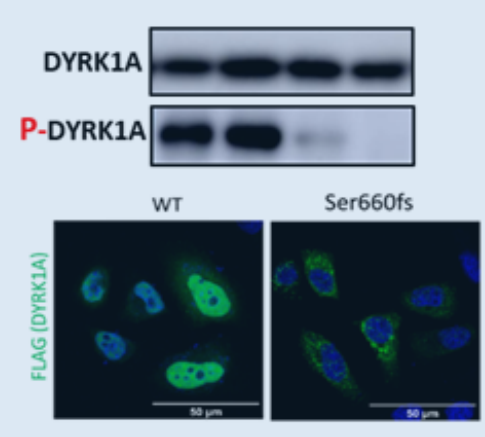

3. Identification of a blood specific episignature

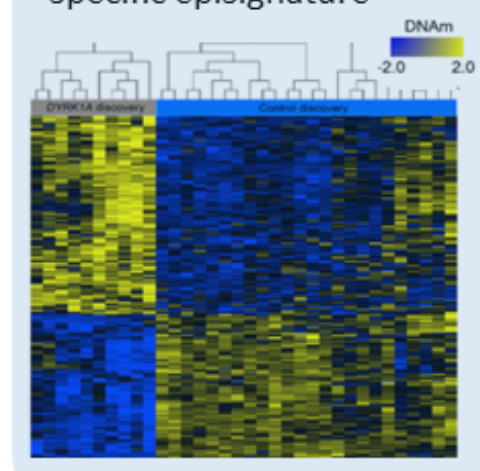

Conclusion: Integration of the different tools to interpret variants in DYRK1A

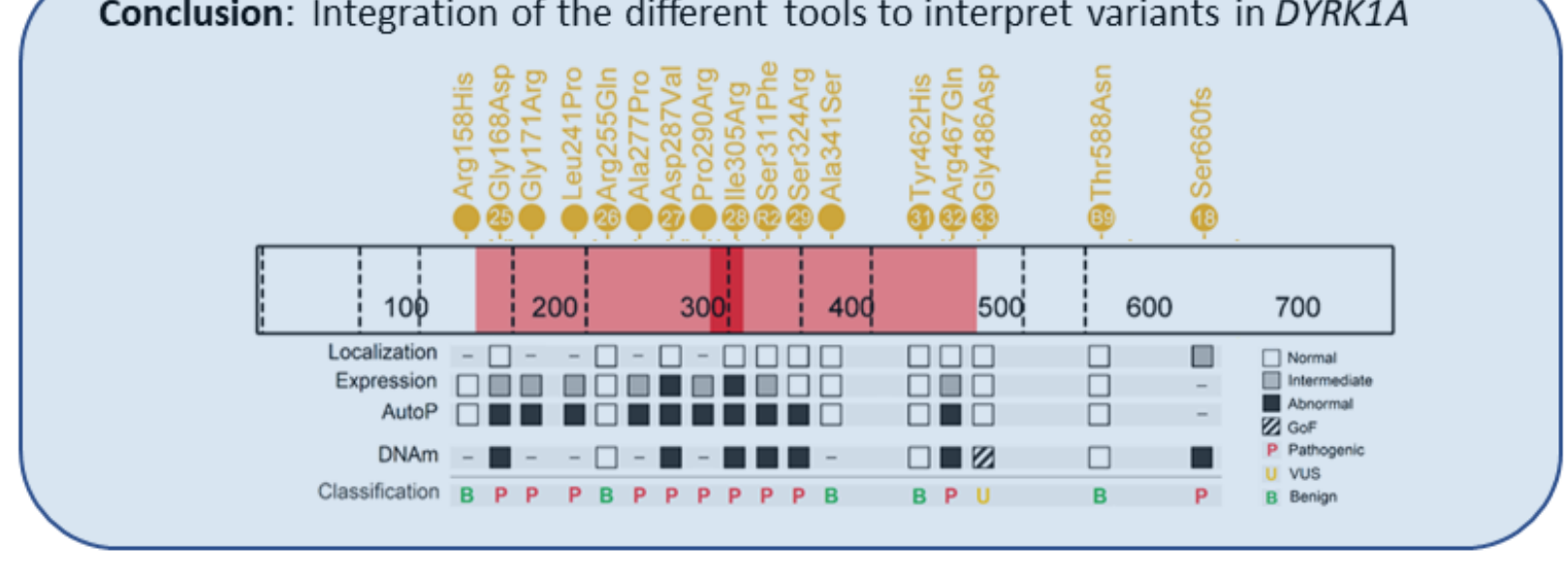

\section{INTRODUCTION}

Intellectual disability (ID) and autism spectrum disorder (ASD) are two highly heterogeneous groups of neurodevelopmental disorders (NDD) with substantial genetic contributions which overlap strongly both at the clinical and genetic levels. More than one thousand genes have been implicated in monogenic forms of NDD, with an important contribution of autosomal dominant forms caused by de novo variants ${ }^{1}$. One of these genes, DYRK1A (dual-specificity-tyrosine-phosphorylation-regulated-kinase-1A) ${ }^{2}$, located on chromosome 21 , is among the genes the most frequently mutated in individuals with ID ${ }^{1}$.

The first DYRK1A disruptions were identified in individuals with intrauterine growth restriction (IUGR), primary microcephaly and epilepsy ${ }^{3}$. Few years later, the first frameshift variant was described in a patient with similar features ${ }^{4}$. The clinical spectrum associated with $D Y R K 1 A$ pathogenic variants (MRD7 for Mental Retardation 7 in OMIM) was further refined with the publication of additional patients, presenting suggestive facial dysmorphism, severe speech impairment and feeding difficulty, while epilepsy and prenatal microcephaly were not always present ${ }^{5-9}$. Pathogenic variants were also identified in cohorts of individuals with ASD ${ }^{10}$, but all have ID ${ }^{11}$. The DYRK1A gene encodes a dual tyrosine-serine/threonine (Tyr-Ser/Thr) kinase composed of a central catalytic domain including Tyrosine-321, involved in DYRK1A activation by autophosphorylation ${ }^{12}$, two nuclear localization signal sequence (NLS), and additional functional domains. DYRK1A is ubiquitously expressed during embryonic development and in adult tissues. Its location is both cytoplasmic and nuclear and varies by cell type and stage of development ${ }^{13}$. By the 
number and diversity of its proposed protein targets, DYRK1A regulates numerous cellular functions (reviews ${ }^{14,15}$ ), among them the MAPT (Tau) protein phosphorylated by DYRK1A on its Thr212 position ${ }^{16}$.

High Throughput Sequencing (HTS) has revolutionized the identification of genetic variants for diagnostic applications but a major challenge remains in the interpretation of the vast number of variants, especially for highly heterogeneous disease such ID. A combination of genetic, clinical and functional approaches, summarized by the American College of Medical Genetics and the Association for Molecular Pathology (ACMG/AMP), are commonly used to interpret these variants ${ }^{17}$. A significant proportion of the variants, especially the missense variants, remain classified as variants of unknown significance (VUS, according to ACMG/AMP) after the primary analysis. For autosomal dominant diseases with complete penetrance such as DYRK1A syndrome, the de novo occurrence of a variant is a strong but not absolute argument for pathogenicity, and the parents' genotype is not always available. Clinical observations are useful but could also lead to misinterpretation, as numerous ID-associated manifestations are unspecific. Many tools have been developed to predict in silico the pathogenicity of missense variants but they remain predictions and protein-specific functional tests are useful to confirm variants effect. DNA methylation (DNAm) is also a powerful tool to test variant pathogenicity in disorders associated with epigenetic regulatory genes. We discovered that pathogenic variants in these genes can exhibit disorder-specific DNAm signatures comprised of consistent, multilocus DNAm alterations in peripheral blood, useful for classifying variants in these genes as pathogenic or benign ${ }^{18-21}$. DYRK1A has numerous targets, and while it is not well-described as an epigenetic regulator, it has been shown to phosphorylate histone H3 and histone acetyltransferase proteins ${ }^{22,23}$. Therefore, we hypothesized that pathogenic variants in DYRK1A might generate a specific DNAm signature in blood.

We reviewed the clinical signs present in 34 individuals never reported and carrying deletions or clearly pathogenic variants in DYRK1A to refine the clinical spectrum of DYRK1A syndrome. Based on these data, we developed a score to help to recognize affected individuals and to interpret variants (reverse phenotyping). In parallel, we developed in silico and in vitro approaches to assess variant effects on DYRK1A function. Finally, we defined a DNAm signature specific to DYRK1A syndrome in patient blood. We used this multifaceted approach to interpret 17 variants identified in patients with ID/NDD and demonstrated its efficiency in optimizing accurate interpretation of variants.

\section{METHODS}

\section{Patients and molecular analysis}

Variants in DYRK1A (NM_001396.4) were identified during routine genetic analyses in individuals referred to clinical genetic services for intellectual disability in France, Denmark and Switzerland: CGHarray, DYRK1A coding regions sanger sequencing, targeted next generation sequencing of ID genes (TES) ${ }^{24-}$ ${ }^{26}$, trio or simplex clinical exome sequencing (CES) or exome sequencing (ES), and confirmed by an additional method. Combined Annotation Dependent Depletion (CADD) and Nnsplice were used to predict the effect of missense and splice variants respectively. Fibroblasts were established from skin biopsies for Ind \#1, \#11, \#22, \#24, Bronicki\#9 and Bronicki\#10 and cultivated as previously described ${ }^{27}$. Paxgene blood samples were collected for Ind \#9, \#18, \#19 and \#30. mRNA extraction, RNA sequencing, RT-PCR or qPCR (primers available on request) were performed as previously described ${ }^{28}$. 


\section{Phenotypic analysis and clinical scoring}

Clinical information and photographs were provided by the referring clinicians for the 44 individuals reported here (Table S1) as well as for six individuals previously published (individuals Bronicki\#2, \#3, \#8, $\# 9$ and \#10 and Ruaud\#2) $)^{6,7}(n=50$ individuals in total). Based on the most frequent signs and the morphometric characteristics presented by the 34 individuals with truncating variants in DYRK1A, a clinical score out of 20 was established (DYRK1A_I, $n=21$ individuals with photographs available Table S2). Clinical scores were calculated for this initial cohort plus a second cohort (replication cohort, DYRK1A_R, $\mathrm{n}=13$ ) with individuals already described in previous publications ${ }^{5-7}$ by experienced clinical geneticists, as well as for individuals with other frequent monogenic forms of ID, caused by pathogenic variants in $D D X 3 X$ $(\mathrm{n}=5), \operatorname{ANKRD11}(\mathrm{n}=5), \operatorname{ARID1B}(\mathrm{n}=8), \operatorname{KMT} 2 A(\mathrm{n}=6), \operatorname{MED13L}(\mathrm{n}=5)$, SHANK3 $(\mathrm{n}=6)$ or TCF4 $(\mathrm{n}=6)$ genes.

\section{Definition of sets of missense variants and conservation analysis}

To evaluate which tools are pertinent to predict effect of missense variants on the DYRK1A protein, we used different sets of variants : 1) variants presumed to be benign (negative $N$-set, $n=115$ ) including missense variants reported "benign" in ClinVar plus variants found more than once in GnomAD (november 2019 release), 2) variants reported as “pathogenic”/“likely pathogenic” in Clinvar (positive P-set, n=16), and 3) other variants reported here, in literature, or as VUS in Clinvar (test T-set, n=44)(Table S3). 123 orthologous sequences of human DYRK1A were extracted from the OrthoInspector database version 3.0. and a multiple sequence alignment (MSA) was constructed using the Clustal Omega software. The MSA was then manually refined to correct local alignment errors using the Jalview MSA editor ${ }^{29}$. The refined MSA was used as input to the PROBE software ${ }^{30}$, in order to identify conserved regions in the sequences. The sequences in the MSA were divided into five separate clades: Vertebrates, Metazoans, Protists, Plants and Fungi.

\section{In vitro analysis of variant effect on DYRK1A protein}

DYRK1A expression plasmids (addgene \#101770, Huen lab) were generated from the pMH-SFBDYRK1A vector containing the N-terminal FLAG-tagged human DYRK1A cDNA sequence (NM_001396.4). Variant sequences were obtained by site-directed mutagenesis as described ${ }^{31}$. Antibodies used are listed in Supplementary Methods. HeLa, HEK293 and COS1 cells were maintained and transfected for 24h with DYRK1A plasmids (for immunoflurorescence) or DYRK1A plasmids plus pEGFP-N1 plasmid (for Western Blot) as previously done ${ }^{31,32}$. For autophosphorylation analysis and DCFA7 (WDR68) interaction, proteins were extracted from transfected HEK293 cells and immunoprecipitated with anti-FLAG antibody as described $^{31}$. Phosphorylated Tyr321 DYRK1A was visualized as described ${ }^{33}$ and normalized by the level of total DYRK1A protein. Kinase activity was investigated by co-transfecting DYRK1A and MAPT plasmids (MAPT_OHu28029C_pcDNA3.1(+)-C-HA, geneScript) in HEK293 cells, adapted from ${ }^{34}$. The level of DYRK1A, MAPT and pMAPT (Thr212) proteins were normalized with GAPDH.

\section{DNA methylation signature}

Methylation analysis was performed using blood DNA from individuals with DYRK1A LoF variants $(n=16)$, split into signature discovery $(n=10)$ and validation $(n=6)$ cohorts, based primarily on whether age at time of blood collection was available, and age- and sex-matched neurotypical controls $(n=24)$. Whole blood DNA samples were prepared, hybridized to the Illumina Infinium Human MethylationEPIC BeadChip 
and analyzed as previously described ${ }^{20}$, a total of $n=774,590$ probes were analyzed for differential methylation. Standard quality control metrics showed good data quality for all samples except Ind\#20. Briefly, limma regression with covariates age, sex, and five predicted blood cell types identified a DNAm signature with a Benjamini-Hochberg adjusted $p$-value $<0.05$ and $10 \%$ methylation difference. Next, we developed a support vector machine (SVM) model with linear kernel trained on including non-redundant CpG sites ${ }^{20}$ using the methylation values for the discovery cases vs. controls. The model generated scores ranging between 0 and 1 for tested samples, classifying samples as "positive" (score $>0.5$ ) or "negative" (score $<0.5)$. Additional neurotypical controls $(\mathrm{n}=94)$ and DYRK1A LoF validation samples ( $\mathrm{n}=6$ ) were scored to test model specificity and sensitivity respectively, and samples with pathogenic KMT2A (n=8) and ARID1B $(\mathrm{n}=4)$ variants and DYRK1A missense and distal frameshift $(\mathrm{n}=11)$ variants were tested.

\section{RESULTS}

\section{Identification of genetic variants in DYRK1A in individuals with ID}

We collected molecular and clinical information from 50 individuals with ID (44 never reported and six previously reported ${ }^{6,7}$ ) carrying a variant in DYRK1A identified in clinical and diagnostic laboratories: structural variants deleting or interrupting $D Y R K 1 A$ and recurrent or novel nonsense, frameshift, splice and missense variants (Table 1, Figure S1). When blood or fibroblast samples were available, we characterized the consequences of these variants on DYRK1A mRNA by RNA-sequencing and RT-qPCR (Figure S2, Supplementary Text). For one variant, c.1978del, occurring in the last exon of the gene (Ind \#18), the mutant transcripts escape to nonsense mRNA mediated decay (NMD) and result in a truncated protein p.Ser660fs (or p.Ser660Profs*43) retaining its entire kinase domain (Figure S2F). The variants occurred de novo in most of the cases (36/44), one was inherited from a mosaic father and parental DNA was not available for the seven others.

\section{Clinical manifestations in individuals with pathogenic variants in DYRK1A and definition of a clinical score}

We reviewed the clinical manifestations of the patients with truncating variants, except p.(Ser660fs)(Table S1, Supplementary text). Recurrent features include, consistently with what was reported 5,6,8,11: moderate to severe ID, prenatal or postnatal progressive microcephaly, major speech impairment, feeding difficulties, seizures and especially history of febrile seizures, autistic traits and anxiety, delayed gross motor development with unstable gait, brain MRI abnormalities including dilated ventricles and corpus callosum hypoplasia and recurrent facial features (Figure 1A, Figure S3). We found genital abnormalies as reported ${ }^{35}$ but no obvious renal anomalies. We noted the importance of skin manifestations and especially atopic dermatitis. We used recurrent features to establish a "DYRK1A-clinical score" (CS DYRK1A) on a 20 point scale (Figure 1A), which aims to reflect specificity rather than severity of the phenotype. High scores, ranging from 13 to 18.5 (mean=15.5), were obtained for the individuals having a pathogenic variant in DYRK1A described here (DYRK1A_I) or previously (DYRK1A_R) (Table S2, Figure 1B). The threshold of $C_{\text {DYRK1A }}>=13$ appears to be discriminant between individuals with LoF variants in DYRK1A (all $\geq 13$ ) and individuals suffering from another form of ID (all $<13$ ) and is considered as "highly

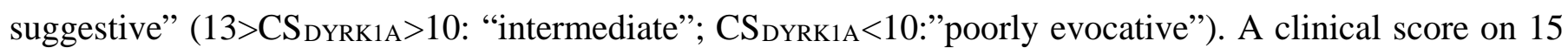
points without photograph is less discriminative (Figure S4). 
We evaluated the discriminative power of the CADD score, commonly used in medical genetics ${ }^{36}$ to interpret missense variants in DYRK1A. If a significant difference in the CADD score distribution is observed between the "benign" variants (N-set, see Methods) and those reported as "pathogenic" (P-set, see Methods)(p-value $<0.0001$ ), a substantial proportion of the $\mathrm{N}$-set variants still have a CADD score above thresholds (20 or 25) usually used to define pathogenicity (Figure S5A). This could be explained by the high degree of amino acid conservation of DYRK1A among vertebrates, and this could lead to over-interpretation of pathogenicity. We performed sequence alignment with orthologs from different taxon (Figure S6) and confirmed that using sequences from vertebrates only is not efficient to classify missense variants, as one third of the N-set variants affect amino acids conserved in all species (Figure S5B, V=100\%). Considering conservation parameters going beyond vertebrates appears more discriminant (13/16 P-set and 1/115 N-set variants are conserved in $100 \%$ of vertebrates, $90 \%$ of metazoan and $80 \%$ of other animals) (Figure S5B).

\section{In vitro characterization of consequences of missense variants on DYRK1A protein}

In order to test the consequences of the missense variants in vitro, we overexpressed wild-type (WT) and mutant DYRK1A proteins in three different cell lines (HEK293, HeLa, COS1) including a truncating pathogenic variant Arg413fs and a benign missense variant from gnomAD, Ala341Ser. A significant and drastic decrease in DYRK1A protein level (Figure 2A), due to a reduction of protein stability (Figure S7A), was observed for the truncating Arg413fs and missense Asp287Val, Ser311Phe, Arg467Gln, Gly168Asp and Ile305Arg variants in each cell type. None of the variants affects DYRK1A interaction with DCAF7 (WDR68) (Figure S7B). To be active, DYRK1A has to undergo an autophosphorylation on Tyrosine 321 12. To measure the level of active DYRK1A protein, we detected phospho-DYRK1A (Tyr321) by immunoprecipitation followed by immunoblot using anti-phospho-HIPK2, as previously described ${ }^{33}$ (Figure 2B). We confirmed that the three variants previously tested (Asp287Val, Ser311Phe and Arg467Gln) abolish autophosphorylation ${ }^{33,37}$, as do the Gly168Ap and Ile305Arg variants. The Ser324Arg DYRK1A variant showed only residual autophosphorylation. No effect on autophosphorylation was observed for Arg255Gln, Tyr462His, Gly486Asp and Thr588Asn. No effect was detected either for the Glu366Asp amino acid change, but the analysis of patient's blood mRNA showed that this variant (c.1098G > T) affects splicing leading to a deletion of 49 amino acids p.Ile318_Glu366del (Figure S2G). We used this strategy to test additional variants reported in databases and showed that Arg158His, affecting a highly conserved amino acid position but reported twice in GnomAD, does not affect DYRK1A protein. Ala277Pro, reported as pathogenic in ClinVar, as well as Gly171Arg, Leu241Pro and Pro290Arg, reported initially as VUS in ClinVar, affect both DYRK1A level and autophosphorylation (Figure S8A-B, Table S3, Figure S5B). None of the missense variants appears to affect DYRK1A cellular localization, contrary to Arg413fs variant or changes in NLS domains (Supplementary Text, Figure S8C). However, we observed an aggregation of DYRK1A proteins with the distal frameshift variant Ser660fs (Figure 2C), preventing a correct measure of the protein and autophosphorylation levels.

\section{Identification of a DNAm signature associated with DYRK1A pathogenic variants}

To determine if DYRK1A is associated with specific changes in genome-wide DNA methylation (DNAm) in blood, we used methylation array analysis. We compared DNAm in blood for a subset (discovery) of our cohort carrying pathogenic LoF variants in DYRK1A with age- and sex-matched neurotypical controls and identified $n=402$ differentially methylated CpG sites corresponding to 165 RefSeq genes (Table S4, Figure 3A-B). The sensibility and specificity of the score (0-1) derived from this signature 
was validated using additional individuals with DYRK1A truncating variants (validation), additional controls and individuals with pathogenic variants in other genes (Table S5; Figure 3C). Next, we scored the samples with missense variants and found that six classified positively (p.Asp287Val, p.Ser311Phe, p.Arg467Gln, p.Gly168Asp, p.Ile305Arg and p.Ser324Arg) and three negatively (p.Arg255Gln, p.Tyr462His, p.Thr588Asn) (Figure 3B-C, Table S5, Figure S9). The sample with the distal frameshift variant p.Ser660fs classified as DNAm positive, with a high score (0.92). The sample with the p.Gly486Asp variant clustered out from both DYRK1A cases and controls and its methylation profile was even opposite to DYRK1A LoF cases (increased methylation at sites decreased in LoF cases and vice versa, Figure S9, S11), suggesting this variant might have a gain-of-function (GoF) effect. A notable feature of these GoF CpG sites is that they tended to cluster together, as for instance in the HIST1H3E promoter (Table S4).

\section{Integration of the different tools to reclassify variants}

We integrated the clinical score, in silico predictions, functional assays results and DNAm score to evaluate the pathogenicity of the variants and reclassify them according to ACMG/AMP categories (Figure 4, Table S6). We found that variants p.Gly168Asp, p.Asp287Val, p.Ile305Arg, p.Ser311Phe and

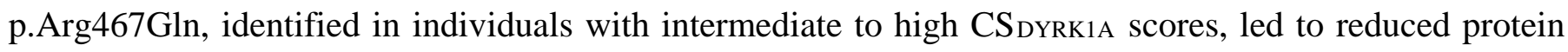
level as well as an absence of autophosphorylation activity, which was previously described for three of them ${ }^{33,37}$. All classified as DNAm-positive, definitively supporting their pathogenicity. For the p.Ser324Arg variant, identified de novo in a patient with an intermediate CS DYRK1A score, we observed only a slight decrease of DYRK1A stability and a partial decrease of its autophosphorylation ability. The binary nature of the DNAm signature, showing a positive score, definitively supports its pathogenic effect.

The p.Arg255Gln and p.Tyr462His variants were identified in individuals with low CS DYRK1A score, they had relatively high CADD score (24 and 29.6) but affect amino acids not highly conserved. They had no effect on protein level, autophosphorylation and cellular localization of DYRK1A and classified DNAmnegative, and were therefore both considered as likely benign. While parental DNA was not available to test the inheritance of p.Arg255Gln, the variant p.Tyr462His occurred de novo. This individual has an affected brother who does not carry the variant, and exome sequencing of the whole family failed to identify additional promising variants, even taken into account the possibility of two different origins for the brothers. This remains puzzling and WGS is ongoing for both to go further. Another de novo variant affecting the same position p.Tyr462Cys was identified in a girl with mild developmental delay, hypotonia and hypermobility without facial dysmorphia, who finally obtained another molecular diagnosis (personal communication Sander Stegmann, Maastricht University Medical Center). The p.Thr588Asn variant, previously reported as likely pathogenic ${ }^{6}$, affects a mildly conserved amino acid and appears to have no effect on mRNA or protein level and function, as described by others ${ }^{33,37}$. To go further, we tested the ability of the mutant Thr588Asn DYRK1A to phosphorylate MAPT on its Thr212 and confirmed it does not affect its kinase activity (Figure S10). Moreover, a knock-in mouse model generated for Thr588Asn failed to present any decrease of kinase activity and any obvious behavioral phenotype (Supplementary Text, Figure S12). In addition, if this variant was not reported in gnomAD, two other amino acid changes are reported at the same position: p.Thr588Pro, (44 times including once at the homozygote state) and p.Thr588Ala (once). All these arguments plus the fact that the patient's DNAm signature was negative were convincing enough to reclassify this variant as likely benign. The fact that it occurred de novo in a girl with a high CSDYRK1A (15.5/20) remains puzzling, while no additional promising variants were identified in trio-exome sequencing data and no positive classification was found using 20 DNAm signatures available. However, the girl also presents additional manifestations unusual for DYRK1A syndrome such as truncal obesity. 
Only one sample showed a DNAm profile different from both controls and individuals with DYRK1A syndrome (Figure 3A, Figure S9 and S11). This individual has a low CSDYRK1A, presenting relative macrocephaly and ASD without ID and carries a de novo p.Gly486Asp variant. This variant was previously reported in another individual with $\mathrm{NDD}^{38}$, but it was not possible to obtain DNA, clinical or inheritance information. No significant change in protein and autophosphorylation level was observed for this variant and analysis of MAPT Thr212 phosphorylation failed to confirm the potential GoF effect (Figure S10).

We characterized the consequences of a distal frameshift de novo variant, p.Ser660fs. Its overexpression leads to cytoplasmic aggregation of DYRK1A, which makes it difficult to quantify the real effect on protein level, autophosphorylation or kinase activity (Figure S8 and S10). However, its DNAm overlaps those of other individuals with truncating variants located further upstream in the protein, confirming its pathogenic effect (Figure S9). To test if these aggregations could be driven by the novel Cterminal extension added by the frameshift variant (43 amino acids), we introduced nonsense variants at the same position (Ser660* and Ser661*). As no aggregate was detected (Figure 2C \& S8D), we concluded that the C-terminal extension is responsible for the self-aggregation of the mutant DYRK1A protein. Interestingly, the two truncating variants Ser660* and Ser661* did not affect DYRK1A level, localization or autophosphorylation (Figure S8A-B).

\section{DISCUSSION}

Here we report clinical manifestations of 34 novel patients with clear loss-of-function (LoF) variants in DYRK1A, refining the clinical spectrum associated with DYRK1A syndrome. We used recurrent signs present in individuals to establish a clinical score, which may seem outdated in the era of pangenomic approaches but is in fact very useful to interpret variants of unknown significance identified by these approaches. Indeed, here we demonstrated that the combination of clinical data together with in silico and in vitro observations are essential to interpret variants accurately.

Since DYRK1A is a highly conserved gene in vertebrates, we assumed that in silico predictive tools using conservation calculated mainly from vertebrates might overestimate the potential pathogenicity of missense variants. We showed that deeper conservation analyses using additional taxa are useful to improve the predictions for missense variants. However, in silico analyses have their limitations, and functional assays are essential to assess variant effect conclusively. We therefore tested the effect of 17 variants and showed that ten of them decreased both DYRK1A protein level and DYRK1A autophosphorylation level. The remaining variants showed no effect on protein function (Figure 4). However, the absence of effects observed during series of functional tests does not totally exclude a potential effect.

Over the past five years, several studies have found patients with specific monogenic disorders involving genes encoding epigenetic regulatory proteins are associated with DNAm signatures in blood. The advantage of such signatures is the high rate of clear classification (positive/pathogenic vs negative/benign) they provide for variants. Considering the potential role played by DYRK1A in epigenetic regulation ${ }^{22,23,39}$, we tested whether DYRK1A LoF leads to such a DNAm profil and identified a DYRK1A DNAm signature with high sensitivity and specificity (Figure 3). We undertook this work in whole-blood (as most signature work is done) due its clinically availability and in silico tools to account for cell proportion differences. We expect many of these changes to be blood-specific in patients with DYRK1A pathogenic variants. However, enough DNAm changes may overlap other tissues for the blood signature to have cross-tissue utility for variant classification, as we found for fibroblasts in Sotos Syndrome ${ }^{18}$. The combination of clinical score (CS DYRK1A), in silico predictions, functional assays and DNAm signature allow to reclassify ten missense 
variants as pathogenic. Three variants were considered as likely benign: a variant located in the catalytic domain whose inheritance was unknown, p.Arg255Gln, and two de novo variants located at the end or outside of this domain: p.Tyr462His and p.Thr588Asn.

Still based on methylation data, we suspected a gain-of-function (GoF) effect for another de novo variant located outside the catalytic domain: p.Gly486Asp. We have already shown that DNAm profiles at gene-specific signature sites provide a functional readout of each variants effect, GoF activity. Indeed, in previous work, we found the same pattern for a patient with a missense variant in EZH2, typically associated with Weaver syndrome. The patient, presenting undergrowth rather than overgrowth characteristic of Weaver syndrome, had an opposite DNAm profile to EZH2 cases relative to controls and carried a missense variant which was demonstrated to increase EZH2 activity ${ }^{19}$. In our case, we could not confirm the putative GoF effect of Gly486Asp by measuring MAPT-Thr212 phosphorylation. Arranz et al. observed on the contrary an increase of DYRK1A kinase activity ${ }^{37}$ for this variant, but they reported significant increase for five additional variants, including one present four times in GnomAD (Arg528Trp), which might question the sensitivity of the test.

We identified a de novo distal frameshift variant in the last exon of DYRK1A leading to DYRK1A aggregation in vitro, which needs to be confirmed in vivo. Interestingly, nonsense changes introduced at this position (aa660 and 661) lead to the expression of a protein which seems to be stable, does not aggregate, and maintains its autophosphorylation capacity and ability to phosphorylate MAPT (Figure 2C, S8C-D, S10). Therefore, we think that distal truncating variants should be interpreted with caution, especially when they escape to NMD. Three additional such distal variants are reported in individuals with ID/NDD in ClinVar/literature (Table S8) for which DNAm analysis on blood samples would be interesting to perform.

In conclusion, we developed a combination of tools efficient to interpret variants identified in DYRK1A. We showed that missense variants located outside and inside the catalytic domain as well as variants leading to distal premature stop codon are not necessarily pathogenic. These results illustrate that variants in DYRK1A, as well as in other NDD causative genes, should be interpreted with caution, even if they occur de novo. In the future, we recommend performing DNAm analysis if blood DNA sample is available or, if not, in vitro testing of variant effect on DYRK1A autophosphorylation.

\section{DATA AVAILABILITY}

Variants were submitted to ClinVar or to Decipher database (as indicated in Table 1). Additional data are available upon request.

\section{ACKNOWLEDGEMENTS}

The authors would like to thank the families for their participation and support. The authors also thank the Agence de Biomédecine, Fondation APLM, Fondation Maladies Rares and Fondation Jérome Lejeune for financial support. We also thank the Centre National de Génotypage, the diagnostic laboratories of Hôpitaux Universitaire de Strasbourg (HUS), clinical genetic residents, the GenomEast sequencing and the molecular biology platforms of IGBMC.

\section{CONFLICTS OF INTEREST}

None 


\section{ETHICS DECLARATION}

Individuals were referred by clinical geneticists for genetic testings as part of routine clinical care. All patients enrolled and/or their legal representative have signed informed consent for research use and authorization for publication, including for photograph publication for those included in Figure S3. All the institutions received local IRB approval to use these data in research purpose. The main IRB approval was obtained from the Ethics Committee of the Strasbourg University Hospital (CCPPRB). For experiments performed in animal, they were all done in compliance with the ARRIVE Guidelines and were non-invasive procedures approved by Com'Eth, the local ethic committee.

\section{WEB RESSOURCES}

The URLs for online tools and data presented herein are:

CADD: https://cadd.gs.washington.edu/

ClinVar: http://www.ncbi.nlm.nih.gov/clinvar/

Clustal Omega: https://www.ebi.ac.uk/Tools/msa/clustalo/

dbSNP: http://www.ncbi.nlm.nih.gov/projects/SNP/

Decipher: https://decipher.sanger.ac.uk/

GnomAD: http://gnomad.broadinstitute.org/

Integrative Genomics Viewer (IGV): http://www.broadinstitute.org/igv/

Mutation Nomenclature: http://www.hgvs.org/mutnomen/recs.html

Nnsplice: https://www.fruitfly.org/seq_tools/splice.html

OMIM: http://www.omim/org/

UCSC: http://genome.ucsc.edu/

CADD score: https://cadd.gs.washington.edu/

OrthoInspector database: https://www.lbgi.fr/orthoinspectorv3/databases

\section{BIBLIOGRAPHY}

1. Deciphering Developmental Disorders Study. Prevalence and architecture of de novo mutations in developmental disorders. Nature. 2017;542(7642):433-438. doi:10.1038/nature21062

2. Gonzalez-Mantilla AJ, Moreno-De-Luca A, Ledbetter DH, Martin CL. A Cross-Disorder Method to Identify Novel Candidate Genes for Developmental Brain Disorders. JAMA Psychiatry. 2016;73(3):275-283. doi:10.1001/jamapsychiatry.2015.2692

3. Møller RS, Kübart S, Hoeltzenbein M, et al. Truncation of the Down syndrome candidate gene DYRK1A in two unrelated patients with microcephaly. Am J Hum Genet. 2008;82(5):1165-1170. doi:10.1016/j.ajhg.2008.03.001

4. Courcet J-B, Faivre L, Malzac $P$, et al. The DYRK1A gene is a cause of syndromic intellectual disability with severe microcephaly and epilepsy. J Med Genet. 2012;49(12):731-736. doi:10.1136/jmedgenet-2012-101251

5. van Bon BWM, Coe BP, Bernier R, et al. Disruptive de novo mutations of DYRK1A lead to a syndromic form of autism and ID. Mol Psychiatry. 2016;21(1):126-132. doi:10.1038/mp.2015.5

6. Bronicki LM, Redin C, Drunat S, et al. Ten new cases further delineate the syndromic intellectual disability phenotype caused by mutations in DYRK1A. Eur J Hum Genet. 2015;23(11):1482-1487. doi:10.1038/ejhg.2015.29

7. Ruaud L, Mignot C, Guët A, et al. DYRK1A mutations in two unrelated patients. Eur J Med Genet. 2015;58(3):168-174. doi:10.1016/j.ejmg.2014.12.014

8. Ji J, Lee $\mathrm{H}$, Argiropoulos B, et al. DYRK1A haploinsufficiency causes a new recognizable syndrome with microcephaly, intellectual disability, speech impairment, and distinct facies. Eur J Hum Genet. 2015;23(11):1473-1481. doi:10.1038/ejhg.2015.71

9. Meissner LE, Macnamara EF, D'Souza P, et al. DYRK1A pathogenic variants in two patients with syndromic intellectual disability and a review of the literature. Mol Genet Genomic Med. Published online November 7, 2020:e1544. doi:10.1002/mgg3.1544

10. O'Roak BJ, Vives L, Fu W, et al. Multiplex targeted sequencing identifies recurrently mutated genes in autism spectrum disorders. Science. 2012;338(6114):1619-1622. doi:10.1126/science.1227764

11. Earl RK, Turner TN, Mefford HC, et al. Clinical phenotype of ASD-associated DYRK1A haploinsufficiency. Mol Autism. 2017;8:54. doi:10.1186/s13229-017-0173-5

12. Himpel S, Panzer P, Eirmbter K, et al. Identification of the autophosphorylation sites and characterization of their effects in the protein kinase DYRK1A. Biochem J. 2001;359(Pt 3):497-505. doi:10.1042/0264-6021:3590497 
13. Hämmerle B, Elizalde C, Tejedor FJ. The spatio-temporal and subcellular expression of the candidate Down syndrome gene $\mathrm{Mnb} /$ Dyrk1A in the developing mouse brain suggests distinct sequential roles in neuronal development. Eur J Neurosci. 2008;27(5):1061-1074. doi:10.1111/j.1460-9568.2008.06092.x

14. Tejedor FJ, Hämmerle B. MNB/DYRK1A as a multiple regulator of neuronal development. FEBS J. 2011;278(2):223-235. doi:10.1111/j.1742-4658.2010.07954.x

15. Duchon A, Herault Y. DYRK1A, a Dosage-Sensitive Gene Involved in Neurodevelopmental Disorders, Is a Target for Drug Development in Down Syndrome. Front Behav Neurosci. 2016;10:104. doi:10.3389/fnbeh.2016.00104

16. Woods YL, Cohen P, Becker W, et al. The kinase DYRK phosphorylates protein-synthesis initiation factor elF2Bepsilon at Ser539 and the microtubule-associated protein tau at Thr212: potential role for DYRK as a glycogen synthase kinase 3-priming kinase. Biochem J. 2001;355(Pt 3):609-615. doi:10.1042/bj3550609

17. Richards S, Aziz N, Bale S, et al. Standards and guidelines for the interpretation of sequence variants: a joint consensus recommendation of the American College of Medical Genetics and Genomics and the Association for Molecular Pathology. Genet Med. 2015;17(5):405-424. doi:10.1038/gim.2015.30

18. Choufani S, Cytrynbaum C, Chung BHY, et al. NSD1 mutations generate a genome-wide DNA methylation signature. Nat Commun. 2015;6:10207. doi:10.1038/ncomms10207

19. Choufani S, Gibson WT, Turinsky AL, et al. DNA Methylation Signature for EZH2 Functionally Classifies Sequence Variants in Three PRC2 Complex Genes. Am J Hum Genet. 2020;106(5):596-610. doi:10.1016/j.ajhg.2020.03.008

20. Chater-Diehl E, Ejaz R, Cytrynbaum C, et al. New insights into DNA methylation signatures: SMARCA2 variants in Nicolaides-Baraitser syndrome. BMC Med Genomics. 2019;12(1):105. doi:10.1186/s12920-019-0555-y

21. Aref-Eshghi E, Bend EG, Colaiacovo S, et al. Diagnostic Utility of Genome-wide DNA Methylation Testing in Genetically Unsolved Individuals with Suspected Hereditary Conditions. Am J Hum Genet. 2019;104(4):685-700. doi:10.1016/j.ajhg.2019.03.008

22. Jang SM, Azebi S, Soubigou G, Muchardt C. DYRK1A phoshorylates histone H3 to differentially regulate the binding of HP1 isoforms and antagonize HP1-mediated transcriptional repression. EMBO Rep. 2014;15(6):686-694. doi:10.15252/embr.201338356

23. Li S, Xu C, Fu Y, et al. DYRK1A interacts with histone acetyl transferase p300 and CBP and localizes to enhancers. Nucleic Acids Res. 2018;46(21):11202-11213. doi:10.1093/nar/gky754

24. Redin C, Gérard B, Lauer J, et al. Efficient strategy for the molecular diagnosis of intellectual disability using targeted high-throughput sequencing. J Med Genet. 2014;51(11):724-736. doi:10.1136/jmedgenet-2014-102554

25. Carion N, Briand A, Cuisset L, Pacot L, Afenjar A, Bienvenu T. Loss of the KH1 domain of FMR1 in humans due to a synonymous variant causes global developmental retardation. Gene. 2020;753:144793. doi:10.1016/j.gene.2020.144793

26. Nasser $\mathrm{H}$, Vera L, Elmaleh-Bergès $\mathrm{M}$, et al. CDK5RAP2 primary microcephaly is associated with hypothalamic, retinal and cochlear developmental defects. J Med Genet. 2020;57(6):389-399. doi:10.1136/jmedgenet-2019-106474

27. Balak C, Benard M, Schaefer E, et al. Rare De Novo Missense Variants in RNA Helicase DDX6 Cause Intellectual Disability and Dysmorphic Features and Lead to P-Body Defects and RNA Dysregulation. Am J Hum Genet. 2019;105(3):509-525.

doi:10.1016/j.ajhg.2019.07.010

28. Quartier A, Chatrousse L, Redin C, et al. Genes and Pathways Regulated by Androgens in Human Neural Cells, Potential Candidates for the Male Excess in Autism Spectrum Disorder. Biol Psychiatry. Published online January 9, 2018. doi:10.1016/j.biopsych.2018.01.002

29. Waterhouse AM, Procter JB, Martin DMA, Clamp M, Barton GJ. Jalview Version 2--a multiple sequence alignment editor and analysis workbench. Bioinformatics. 2009;25(9):1189-1191. doi:10.1093/bioinformatics/btp033

30. Kress A, Lecompte O, Poch O, Thompson JD. PROBE: analysis and visualization of protein block-level evolution. Bioinformatics. 2018;34(19):3390-3392. doi:10.1093/bioinformatics/bty367

31. Mattioli F, Isidor B, Abdul-Rahman O, et al. Clinical and functional characterization of recurrent missense variants implicated in THOC6-related intellectual disability. Hum Mol Genet. 2019;28(6):952-960. doi:10.1093/hmg/ddy391

32. Quartier A, Courraud J, Thi Ha T, et al. Novel mutations in NLGN3 causing autism spectrum disorder and cognitive impairment. Hum Mutat. 2019;40(11):2021-2032. doi:10.1002/humu.23836

33. Widowati EW, Ernst S, Hausmann R, Müller-Newen G, Becker W. Functional characterization of DYRK1A missense variants associated with a syndromic form of intellectual deficiency and autism. Biol Open. 2018;7(4). doi:10.1242/bio.032862

34. Lee K-S, Choi M, Kwon D-W, et al. A novel de novo heterozygous DYRK1A mutation causes complete loss of DYRK1A function and developmental delay. Sci Rep. 2020;10(1):9849. doi:10.1038/s41598-020-66750-y

35. Blackburn ATM, Bekheirnia N, Uma VC, et al. DYRK1A-related intellectual disability: a syndrome associated with congenital anomalies of the kidney and urinary tract. Genet Med. 2019;21(12):2755-2764. doi:10.1038/s41436-019-0576-0

36. Kircher M, Witten DM, Jain P, O'Roak BJ, Cooper GM, Shendure J. A general framework for estimating the relative pathogenicity of human genetic variants. Nat Genet. 2014;46(3):310-315. doi:10.1038/ng.2892

37. Arranz J, Balducci E, Arató K, et al. Impaired development of neocortical circuits contributes to the neurological alterations in DYRK1A haploinsufficiency syndrome. Neurobiol Dis. 2019;127:210-222. doi:10.1016/j.nbd.2019.02.022

38. Dang T, Duan WY, Yu B, et al. Autism-associated Dyrk1a truncation mutants impair neuronal dendritic and spine growth and interfere with postnatal cortical development. Mol Psychiatry. 2018;23(3):747-758. doi:10.1038/mp.2016.253

39. Lepagnol-Bestel A-M, Zvara A, Maussion G, et al. DYRK1A interacts with the REST/NRSF-SWI/SNF chromatin remodelling complex to deregulate gene clusters involved in the neuronal phenotypic traits of Down syndrome. Hum Mol Genet. 2009;18(8):1405-1414. doi:10.1093/hmg/ddp047 


\section{FIGURE LEGENDS}

\section{Figure 1. Clinical score for Intellectual Disability associated to DYRK1A haploinsufficiency}

(A) Clinical score out of 20 points established according to the most recurrent clinical features presented by patients (the weight assigned to each symptom being based on its recurrence): clinical symptoms are out of 15 points, while the facial appearance is out of 5 points. EV: enlarged ventricules; $\mathrm{CCA} / \mathrm{H}$ : corpus callosum agenesis or hypoplasia, CA: cerebral atrophy, CeA: cerebellar atrophy (B) Clinical scores calculated for individuals carrying pathogenic variants in DYRK1A reported here and for whom photographs were available ( $\mathrm{n}=21$ )(initial cohort, DYRK1A_I, scores 13-17.5 with a mean of 15.5), the previously published individuals (replication cohort, DYRK1A_R, scores 13.5-18.5, mean=15.3) and the individuals affected with other frequent monogenic forms of ID, associated to variants in ANKRD11, MED13L, DDX3X, ARID1B, SHANK3, TCF4 or KMT2A (scores 3-12.5, mean=7). The clinical score for the individuals carrying missense or distal frameshift variants are indicated in yellow (test); the threshold of CSDYRK1A $>=13$ appeared to be discriminant between individuals with LoF variants in DYRK1A (all $\geq 13$ ) and individuals suffering from another form of ID (all<13). A score above this threshold was therefore considered "highly suggestive". We classified individuals with CS DYRK1A $<10$ as "poorly evokative" and individuals with a CS DYRK1A comprised between 10 and 13 as "intermediate”. Brown-Forsythe and Welsh ANOVA tests with Dunnett's T 3 multiple comparisons test were performed. ns: not significant; ** $\mathrm{p}<0.01$; $* * * \mathrm{p}<0.001$, error bars represent SD.

\section{A}

Frequent features (\% ind.thisreport-prev.reported) Points (/20)

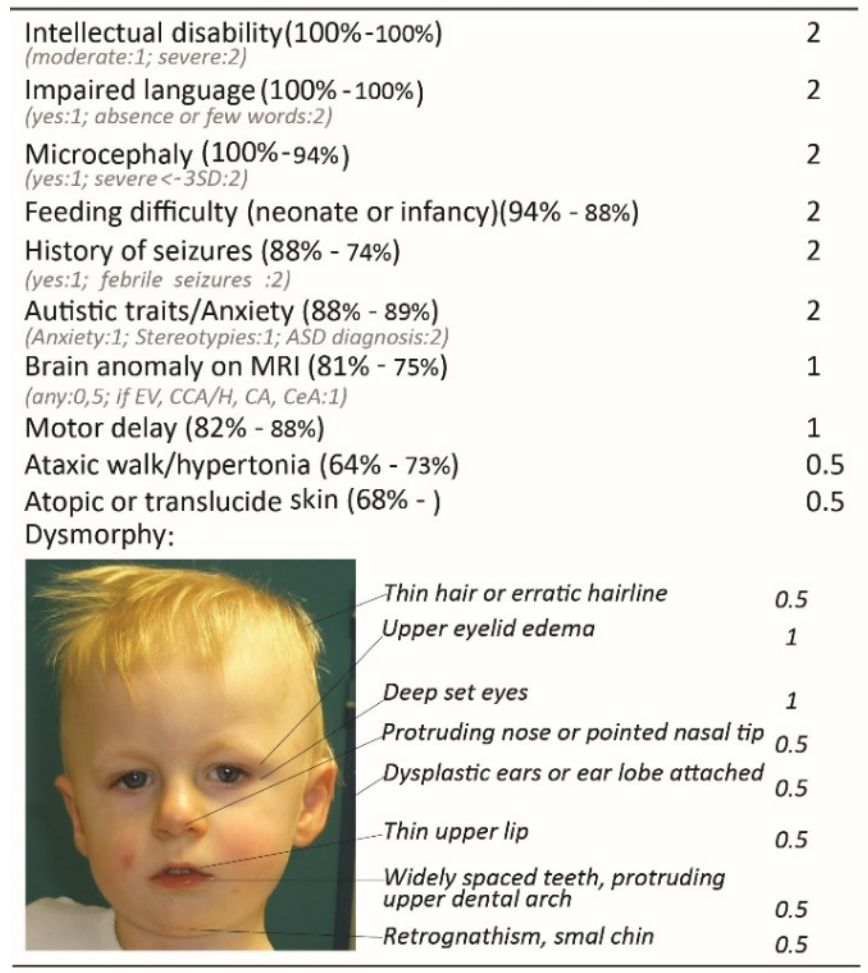

\section{B}
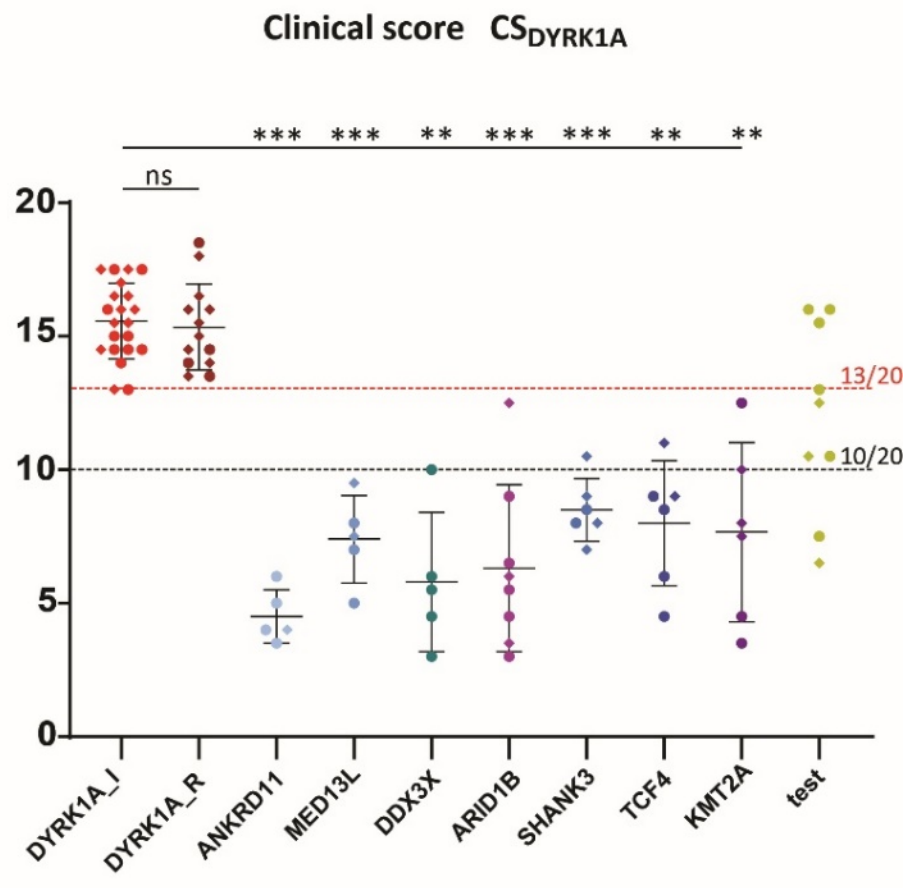


\section{Figure 2. Expression, localization and Tyr321 phosphorylation of DYRK1A mutant proteins}

(A) Level of variant DYRK1A proteins expressed in HeLa, HEK293 and COS cells transiently transfected with DYRK1A constructs. Protein levels were normalized on the level of GFP proteins (expressed from a cotransfected pEGFP plasmid). Quantifications were performed on a total of $n>=9$ series of cells $(n>=3$ Hela cells, n>= 3 HEK293 and n >= 3 COS cells) using ImageJ software. One-way ANOVA with multiple comparison test was performed to compare the level of variant DYRK1A proteins to the level of wild-type DYRK1A protein (orange dashes), applying Bonferroni’s correction: ns: not significant; ${ }^{*} \mathrm{p}<0.05$; ${ }^{* *} \mathrm{p}<$ $0.01 ; * * * \mathrm{p}<0.001$; error bars represent SEM, standard error of the mean; in green, the variant from gnomAD, in red, a truncating variant and in gold, the variants tested in this study (B) DYRK1A's ability to autophosphorylate on Tyr321 was tested in HEK293 cells $(n=3)$ by immunoprecipitations with antiDYRK1A followed by an immunoblot using anti-phospho-HIPK2 as described in Widowati et al. DYRK1A phospho-Tyr321 levels were normalized with DYRK1A total level (orange dashes). Variant DYRK1A phospho-Tyr321 levels were normalized with total DYRK1A protein levels and expressed as percentage of wild-type level. One-way ANOVA test was performed to compare variants to wild-type DYRK1A levels. ns: not significant; ${ }^{* * *} \mathrm{p}<0.001$; error bars represent SEM, standard error of the mean (C) Immunofluorescence experiment showing that Ser660fs (alias Ser660Profs*43) variant leads to DYRK1A protein aggregation when overexpressed in HeLa cells, using a FLAG-tagged DYRK1A proteins carrying Ser660Profs43. No aggregation was observed for the Ser660* variant.

A

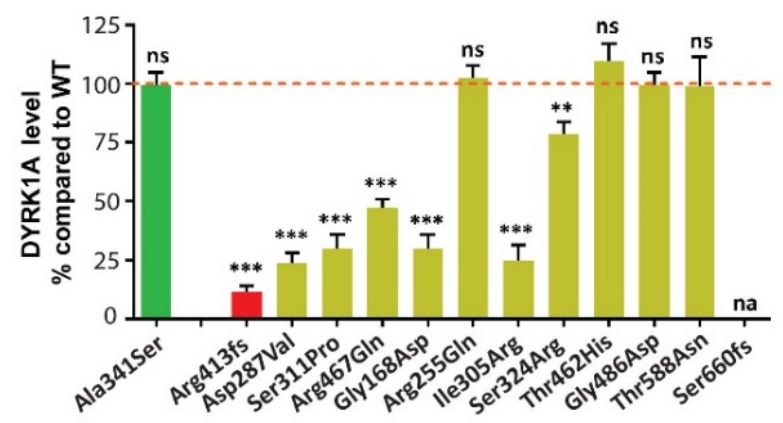

B

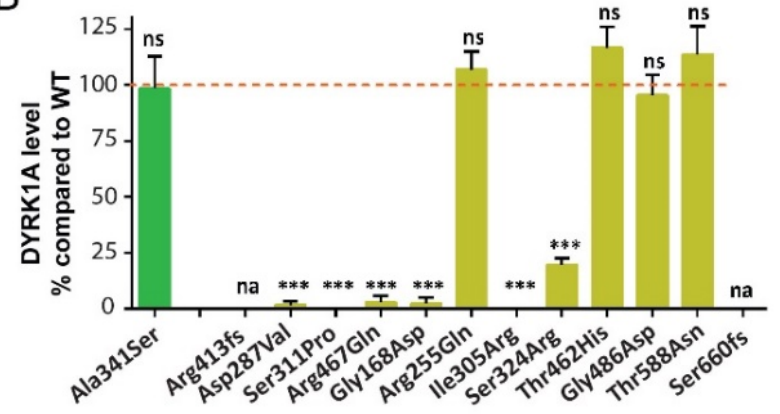

C
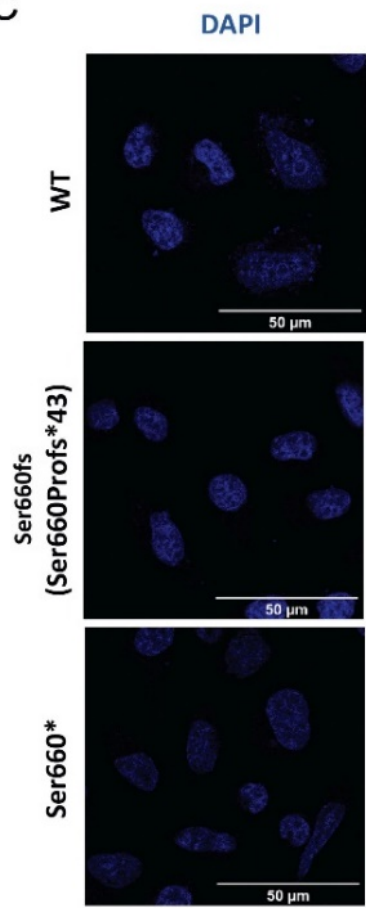
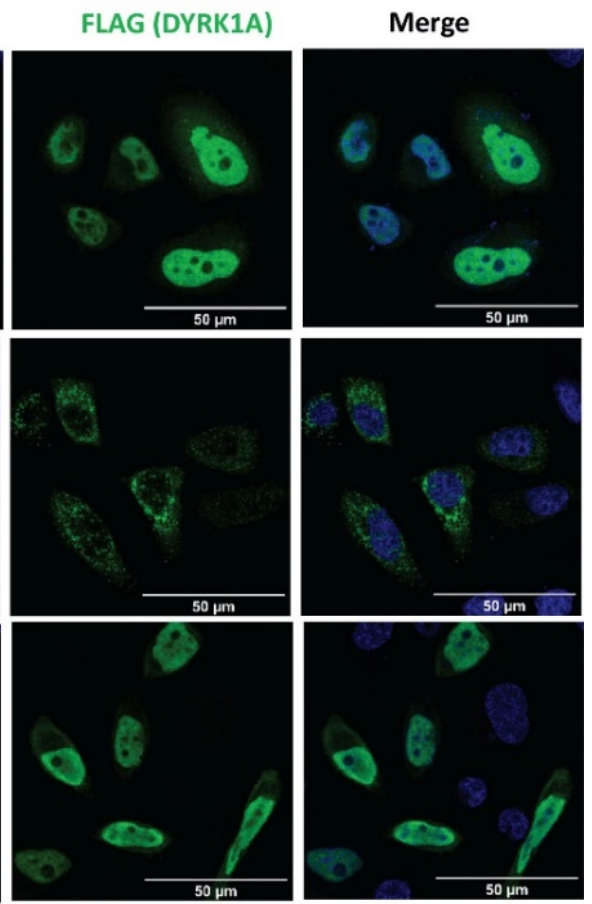
VUS.

(A) Heatmap showing the hierarchical clustering of discovery DYRK1A LoF cases $(\mathrm{n}=10)$ and age- and sexmatched neurotypical discovery controls $(n=24)$ used to identify the 402 differentially methylated signature sites shown. Each row corresponds to a CpG site differentially methylated (DM) and the color gradient represents the normalized DNA methylation value from -2.0 (blue) to 2.0 (yellow) at each site. DNA methylation at these sites clearly separate discovery cases (grey) from discovery controls (blue). Euclidian distance metric is used for the clustering dendrogram. (B) Principal components analysis (PCA) visualizing the DNAm profiles of the study cohort at the 402 signature sites. Validation of DYRK1A LoF cases (not used to define the signature sites; red) cluster with discovery cases, while missense (yellow) and distal LoF (green) variants cluster with either cases or controls. Ind \#33 (Gly486Asp) has an opposite DNAm profile to DYRK1A LoF cases at these sites, suggesting a GoF. (C) Support vector machine (SVM) classification model based on the DNA methylation values in the discovery groups. Each sample is plotted based on its scoring by the model. All samples are clearly positive $(>0.5)$ or negative $(<0.5)$. All DYRK1A validation cases from our cohort $(n=6)$ classified positively, all control validation cases $(n=94)$ classified negatively. Missense variants classified clearly positively or negatively (yellow), the distal frameshift variant (Ind \#18, c.1978del)(green), analyzed in duplicate, classified positively. Pathogenic ARID1B (Coffin-Siris syndrome) and KMT2A (Wiedemann Steiner syndrome) also classified negatively.
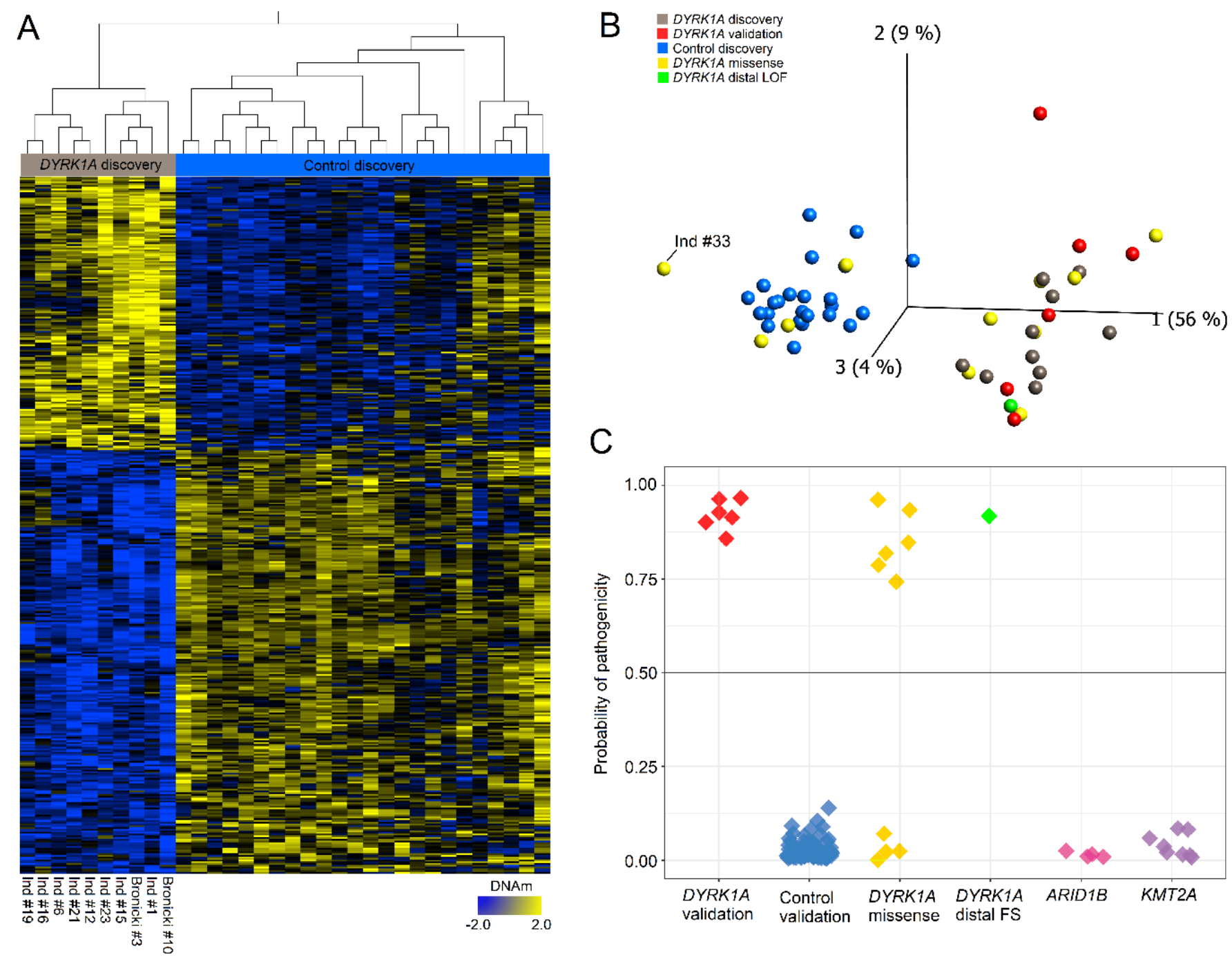
Representation of the DYRK1A protein (the kinase domain is indicated in red and the catalytic domain in dark red) with the positions of the different variants tested with the sample \#ID of the individuals indicated inside the circles. Number: number of individuals with ID reported with the variant; gAD: variant reported in individuals from gnomAD; CS DYRK1A poorly (white), intermediate (grey) or highly (black) evocative, or unknown (-); the white star indicates that the individual presents a high CSDYRK1A score but also additional clinical manifestations unusual for DYRK1A syndrome; CADD below 25 (white), between 25 and 30 (grey) or above 30 (black); conservation: highly conserved $\mathrm{V}=100 \%, \mathrm{M}>90 \%, \mathrm{O}>80 \%$ (black), moderately $\mathrm{V}=100 \%, \mathrm{M}>90 \%, \mathrm{O}<80 \%$ (grey) or midly $\mathrm{V}=100 \% \mathrm{M}<90 \%, \mathrm{O}<80 \%$ (white); Expression or autophosphorylation being normal (white), intermediate decreased (grey), strongly decreased (black); Localization was normal (white), affected (grey) or not tested (-); DNAmethylation positive (black), negative (white), suggestive of a GoF effect (hashed) or not tested (-). Final classification: Pathogenic (P), Benign/Likely benign (B), Unknown significance (U).

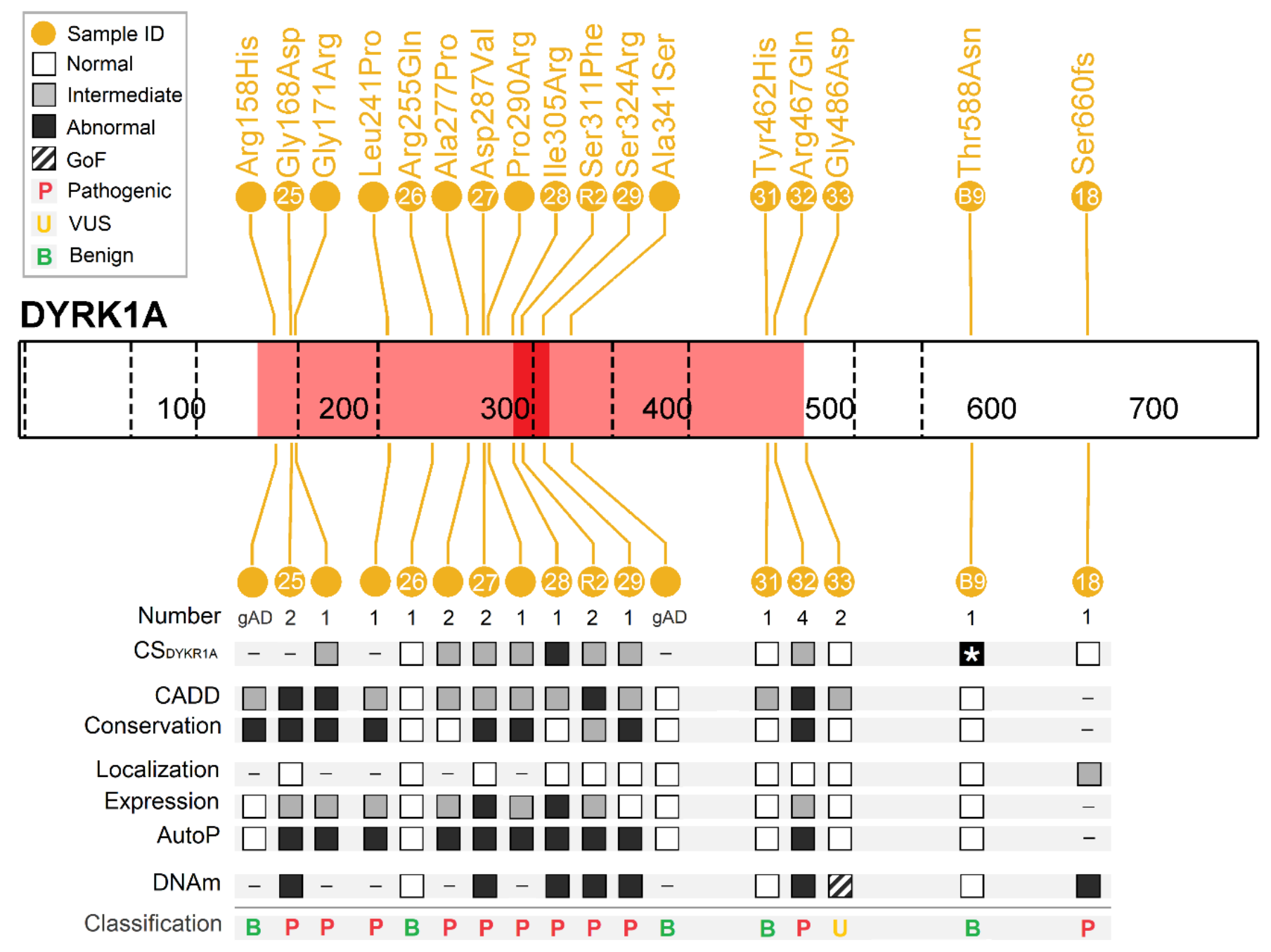


Table 1. List of variants identified in DYRK1A in individuals with intellectual disability

This list includes variants identified in the 44 individuals never reported as well as variants previously reported in six individuals ${ }^{6,7}$ for whom we collected biological samples and additional clinical information. The truncating variants clearly pathogenic are represented in red; the missense variants and the distal frameshift variant to test are in gold. Variants are reported according to standardized nomenclature defined by the reference human genome GRCh37/hg19 and the DYRK1A isoform NM_001396.4; del: deletion of the gene ; Trans.: translocation interrupting the gene ; Ns: nonsense ; Fs: frameshift, Spl: splice ; Ms: missense variants; Infr.: inframe deletion; TES: targeted exome sequencing of ID genes TES ${ }^{1}$ : panel of ID genes from Carion et al. ${ }^{47}$; TES $^{2}$ : panel of ID gene adapted from Redin et al. ${ }^{46}$; TES $^{3}$ : panel of 44 ID genes; TES ${ }^{4}$ : panel of microcephaly genes from Nasser et al. ${ }^{48}$; CES: clinical exome sequencing, ES: exome sequencing, Sanger: Sanger sequencing, CGH-array: comparative genomic hybridization-array. ${ }^{\mathrm{a}}$ : the consequences of c.1098G>T is p.Ile318_Glu366del instead of p.Glu366Asp; M : male ; F : female ; DYRK1A_I : initial cohort used to establish DYRK1A clinical score (CS DYRK1A) ; DYRK1A_R :

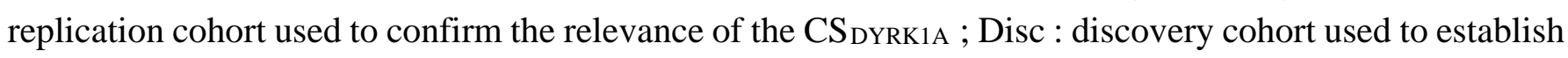
DNA methylation signature (DNAm) ; Valid: validation cohort used to confirm DNA methylation signature (DNAm); test : variants tested for pathogenicity using DNAm. 


\begin{tabular}{|c|c|c|c|c|c|c|c|c|c|c|c|c|c|c|}
\hline \multicolumn{5}{|l|}{ Variant } & \multicolumn{3}{|l|}{ Individual } & \multicolumn{2}{|l|}{ Reporting } & \multicolumn{5}{|c|}{ Analyses performed } \\
\hline GRCh37 (Chr21) & NM_001396.4 & NP_001387.2 & Type & Method & Ind. number & Sex & Inheritance & this individual & $\begin{array}{l}\text { Additional ind. } \\
\text { (ClinVar) }\end{array}$ & $\mathrm{CS}_{\text {DYRK1A }}$ & \begin{tabular}{|l|} 
In \\
silico
\end{tabular} & mRNA & \begin{tabular}{|l} 
in \\
vitro
\end{tabular} & DNAm \\
\hline $\begin{array}{l}\text { g:38481804_40190458del } \\
\text { (DYRK1A ; >10 othergenes) }\end{array}$ & NA & NA & del. & CGH-array & Ind \#1 & $M$ & de novo & NA & NA & DYRK1A_I & - & yes & - & Disc. \\
\hline $\begin{array}{l}\text { g.38722881_39426450del } \\
\text { (DYRK1A; KCNJ2) }\end{array}$ & NA & NA & del. & CGH-array & Ind \#2 & $F$ & de novo & NA & NA & DYRK1A_I & - & - & - & - \\
\hline $\begin{array}{l}\text { g.38302140_40041414del } \\
\text { (DYRK1A; >5 other genes) }\end{array}$ & NA & NA & del. & CGH-array & Ind \#40 & $M$ & de novo & NA & NA & DYRK1A_I & - & - & - & - \\
\hline $\mathrm{t}(9 ; 21)(p 12 ; q 22)$ & between exon 2 \& 3 & NA & trans. & CGH-array & Ind \#3 & M & de novo & NA & NA & DYRK1A_I & - & - & - & - \\
\hline \multirow{3}{*}{ g.38852961C>T } & \multirow{3}{*}{ c. $349 \mathrm{C}>\mathrm{T}$} & \multirow{3}{*}{ p.Arg117* } & \multirow{3}{*}{ Ns } & ES & Ind \#4 & $\mathrm{M}$ & NA & NA & \multirow{3}{*}{$(5 x)$ vcv000373087 } & DYRK1A_I & - & - & - & - \\
\hline & & & & ES & Ind \#5 & $F$ & father mosaic & NA & & DYRK1A_I & - & - & - & - \\
\hline & & & & ES & Ind \#6 & $M$ & de novo & NA & & DYRK1A_I & - & - & - & Disc. \\
\hline \multirow[t]{2}{*}{ g. $38858865 C>T$} & \multirow[t]{2}{*}{ c. $613 \mathrm{C}>\mathrm{T}$} & \multirow[t]{2}{*}{ p.Arg205* } & \multirow[t]{2}{*}{ Ns } & $\mathrm{TES}^{2}$ & Bronicki_\#2 & $M$ & de novo & \begin{tabular}{|l|} 
PMID: 25920557 \\
(ClinVar_SCV000281731= \\
SCV000196058) \\
\end{tabular} & \multirow[t]{2}{*}{ (x7) vcv000162153 } & DYRK1A_R & - & - & - & - \\
\hline & & & & $\mathrm{TES}^{4}$ & Ind \#44 & $M$ & NA & ClinVar_scv001432338 & & DYRK1A_I & - & - & - & - \\
\hline \multirow{2}{*}{ g. $38862575 C>T$} & \multirow{2}{*}{ c. $763 \mathrm{C}>\mathrm{T}$} & \multirow{2}{*}{ p.Arg255* } & \multirow{2}{*}{ Ns } & TES $^{1}$ & Ind \#7 & $M$ & de novo & ClinVar_scv001712097 & \multirow{2}{*}{ (5x) vcv000162152 } & DYRK1A_I & - & - & - & Valid. \\
\hline & & & & NA & Ind \#43 & $F$ & NA & ClinVar_scv001432353 & & DYRK1A_I & - & - & - & - \\
\hline g.38862611C>T & c. $799 \mathrm{C}>\mathrm{T}$ & p.G $\ln 267^{*}$ & Ns & ES & Ind\# 35 & $M$ & de novo & Clinvar_suB9815736 & no & DYRK1A_I & - & - & - & - \\
\hline g. $38862748 \mathrm{~T}>\mathrm{A}$ & c. $936 \mathrm{~T}>\mathrm{A}$ & p.Cys312* & Ns & CES & Ind \#37 & $F$ & de novo & Clinvar_scv001712105 & no & DYRK1A_I & & & & \\
\hline g. $38877655 C>T$ & c. $1309 \mathrm{C}>\mathrm{T}$ & p.Arg $437 *$ & Ns & $\mathrm{TES}^{4}$ & Ind \#34 & $F$ & not in mother & NA & (7x) vcv000162158 & DYRK1A_I & - & - & - & - \\
\hline g. $38877745 C>T$ & c. $1399 \mathrm{C}>\mathrm{T}$ & p.Arg $467^{*}$ & Ns & $\mathrm{TES}^{4}$ & Ind\#42 & $F$ & de novo & ClinVar_scv001432351 & (3x) vcv000204005 & DYRK1A_I & - & - & - & - \\
\hline g.38850510dup & c. 235 dup & p.Arg79fs & Fs & $\mathrm{TES}^{4}$ & Ind \#41 & $F$ & de novo & ClinVar_scv001432470 & no & DYRK1A_I & - & - & - & - \\
\hline g.38850565_38850566del & c.290_291 del & p.Ser97fs & Fs & CES & Ind \#8 & $M$ & de novo & ClinVar_scv001712106 & (2x) vcv000418949 & DYRK1A_I & - & - & - & - \\
\hline g.38850572_38850576del & c.297_301del & p.Leu100fs & Fs & NA & Ind \#9 & $M$ & de novo & ClinVar_scv000485020 & no & DYRK1A_I & - & - & - & - \\
\hline g.38853089del & c.477del & p.Tyr159* & Fs & ES & Ind \#10 & $F$ & de novo & Clinvar_scv001712094 & no & DYRK1A_I & - & - & - & - \\
\hline g.38862514_38862515del & c.702_703del & p.Cys235fs & Fs & ES & Ind \#11 & $M$ & de novo & ClinVar_scv000965742 & no & DYRK1A_I & - & yes & - & Valid. \\
\hline g.38858873_38858876delinsGAA & c.621_624 delinsGAA & p.Glu208fs & Fs & $\mathrm{TES}^{2}$ & Bronicki_\#3 & M & de novo & \begin{tabular}{|l|} 
PMID: 25920557 \\
(ClinVar_SCV000281736 = \\
SCV000196059)
\end{tabular} & no & DYRK1A_R & - & - & - & Disc. \\
\hline g.38862594del & c.782del & p.Leu261fs & Fs & TES $^{2}$ & Ind \#12 & $\mathrm{F}$ & de novo & ClinVar_scv001437790 & no & DYRK1A_I & - & - & - & Disc. \\
\hline g.38862656dup & c.844dup & p.Ser282fs & Fs & $\mathrm{TES}^{1}$ & Bronicki_\#8 & M & de novo & $\begin{array}{l}\text { PMID: } 25920557 \\
\text { (ClinVar_SCV000196064) }\end{array}$ & no & DYRK1A_R & - & - & - & - \\
\hline g.38865371del & c.1004del & p.Gly335fs & Fs & TES $^{1}$ & Ind \#13 & $F$ & not in mother & Clinvar_scv001712087 & no & DYRK1A_I & - & - & - & Valid. \\
\hline g.38865375dup & c.1008dup & p.Pro337fs & Fs & ES & Ind \#14 & $F$ & de novo & Decipher_351807 & no & DYRK1A_I & - & - & - & - \\
\hline g.38865400del & c.1033del & p.Trp345fs & Fs & CES & Ind \#15 & $F$ & de novo & Clinvar_scv001712108 & no & DYRK1A_I & - & - & - & Disc. \\
\hline
\end{tabular}

\section{ACCEPTED MANUSCRIPT - CLEAN COPY}




\begin{tabular}{|c|c|c|c|c|c|c|c|c|c|c|c|c|c|c|}
\hline g.38868553dup & c.1232dup & p.Arg413fs & Fs & TES $^{2}$ & Bronicki_\#10 & $\mathrm{F}$ & de novo & $\begin{array}{l}\text { PMID: } 25920557 \\
\text { (ClinVar_ScV000196066) }\end{array}$ & no & DYRK1A_R & - & yes & yes & Disc. \\
\hline g.38877616del & c.1270del & p.His424fs & Fs & ES & Ind \#39 & $\mathrm{F}$ & de novo & Clinvar_scv001712090 & no & DYRK1A_I & - & - & - & - \\
\hline g.38877679dup & c.1333dup & p.Thr445fs & Fs & ES & Ind \#16 & $\mathrm{F}$ & de novo & Clinvar_scv000778256 & no & DYRK1A_I & - & - & - & Disc. \\
\hline g.38877837del & c.1491delC & p.Ala498fs & Fs & ES & Ind \#17 & $\mathrm{F}$ & de novo & Clinvar_scv000494645 & SCV000056592 & DYRK1A_I & - & - & - & Valid. \\
\hline g.38884520del & c.1978del & \begin{tabular}{|l|} 
p.Ser660fs $=$ \\
p.Ser660Profs $* 43$
\end{tabular} & Fs & TES $^{1}$ & Ind \#18 & $\mathrm{F}$ & de novo & Clinvar_scv001712088 & no & test & - & yes & yes & test \\
\hline g.38852939G>T & c. $328-1 \mathrm{G}>\mathrm{T}$ & p.? & Spl. & TES $^{2}$ & Ind \#19 & $\mathrm{F}$ & de novo & Clinvar_scv001437768 & no & DYRK1A_I & - & yes & - & Disc. \\
\hline g. $38862475 A>G$ & c. $665-2 A>G$ & p.? & Spl. & Sanger & Ind \#20 & $M$ & de novo & Clinvar_scv001712086 & SCV000492145 & DYRK1A_I & - & - & - & Valid. \\
\hline \multirow{2}{*}{ g.38862468_38862472del } & \multirow{2}{*}{ c.665-9_665-5del } & \multirow{2}{*}{ p.? } & Spl. & Sanger & Ind \#21 & $M$ & de novo & \multirow{2}{*}{ Clinvar_scv001437772 } & \multirow{2}{*}{ SCV000677027 } & DYRK1A_I & - & - & - & Disc. \\
\hline & & & Spl. & TES $^{2}$ & Ind \#36 & $M$ & de novo & & & DYRK1A_I & - & - & - & - \\
\hline g. $38862764 G>C$ & $951+1 \mathrm{G}>\mathrm{C}$ & p.? & Spl. & TES $^{1}$ & Ind \#38 & $M$ & de novo & Clinvar_scv001712091 & no & DYRK1A_I & - & - & - & Valid. \\
\hline g.38862767_38862770del & c.951+4_951+7del & p.? & Spl. & ES & Ind \#22 & M & de novo & Clinvar_scvo00965731 & ScV000709803 & DYRK1A_I & - & yes & - & - \\
\hline g. $38877584 A>G$ & c. $1240-2 A>G$ & $p ?$ & Spl. & ES & Ind \#23 & M & de novo & Clinvar_scv000966166 & no & DYRK1A_I & - & - & - & Disc. \\
\hline g.38877585_38877586insTAA & c.1240-1_1240insTAA & p.Glu414* & Spl. & $\mathrm{TES}^{4}$ & Ind \#24 & $\mathrm{F}$ & de novo & Clinvar_scv001432455 & no & DYRK1A_I & - & yes & - & - \\
\hline g. $38853115 \mathrm{G}>\mathrm{A}$ & c. $503 \mathrm{G}>\mathrm{A}$ & p.Gly168Asp & Mis. & TES $^{1}$ & Ind \#25 & $\mathrm{F}$ & de novo & Clinvar_scv001712092 & SCV000573105 & test & yes & - & yes & test \\
\hline g. $38862576 \mathrm{G}>\mathrm{A}$ & c. $764 \mathrm{G}>\mathrm{A}$ & p.Arg255Gln & Mis. & TES $^{1}$ & Ind \#26 & $\mathrm{F}$ & NA & Clinvar_scv001712093 & no & test & yes & - & yes & test \\
\hline g.38862672A>T & c. $860 \mathrm{~A}>\mathrm{T}$ & p.Asp287Val & Mis. & ES & Ind \#27 & M & de novo & Clinvar_scv000598121 & SCV001446739 & test & yes & - & yes & test \\
\hline g.38862726T>G & c. $914 \mathrm{~T}>\mathrm{G}$ & p.lle305Arg & Mis. & Sanger & Ind \#28 & $\mathrm{F}$ & de novo & Clinvar_scv001712110 & no & test & yes & - & yes & test \\
\hline g. $38862744 \mathrm{C}>\mathrm{T}$ & c. $932 C>T$ & p.Ser311Phe & Mis & NA & Ruaud_\#2 & M & de novo & $\begin{array}{l}\text { PMID: } 25641759 \\
\text { (Clinvar_SCV000586742) }\end{array}$ & SCV000520979 & test & yes & - & yes & test \\
\hline g.38865339T>A & c. $972 T>A$ & p.Ser324Arg & Mis. & TES $^{3}$ & Ind \#29 & M & de novo & Clinvar_scv000902439 & no & test & yes & - & yes & test \\
\hline g. $38865465 \mathrm{G}>\mathrm{T}$ & c. $1098 \mathrm{G}>\mathrm{T}^{\mathrm{a}}$ & p.Ile318_Glu366del & Infr. & ES & Ind \#30 & $\mathrm{F}$ & de novo & Decipher_434484 & no & test & yes & yes & yes & - \\
\hline g.38877730T>C & c. $1384 \mathrm{~T}>\mathrm{C}$ & p.Tyr462His & Mis. & $\mathrm{TES}^{2}$ & Ind \#31 & $M$ & de novo & Clinvar_scv001437769 & no & test & yes & - & yes & test \\
\hline g. $38877746 \mathrm{G}>\mathrm{A}$ & c. $1400 \mathrm{G}>\mathrm{A}$ & p.Arg467Gln & Mis. & $\mathrm{TES}^{2}$ & Ind \#32 & $\mathrm{F}$ & de novo & Clinvar_scv001437771 & (3x) vcv000209150 & test & yes & - & yes & test \\
\hline g. $38877803 \mathrm{G}>\mathrm{A}$ & c. $1457 \mathrm{G}>\mathrm{A}$ & p.Gly486Asp & Mis. & ES & Ind \#33 & M & de novo & Clinvar_scv000747759 & no & test & yes & - & yes & test \\
\hline g. $38884305 C>A$ & c. $1763 C>A$ & p.Thr588Asn & Mis & ES & Bronicki_\#9 & $\mathrm{F}$ & de novo & $\begin{array}{l}\text { PMID: } 25920557 \\
\text { (ClinVar_scvo00965705= } \\
\text { scV0o0196065) }\end{array}$ & no & test & yes & yes & yes & test \\
\hline
\end{tabular}

\section{ACCEPTED MANUSCRIPT - CLEAN COPY}




\section{SUPPLEMENTARIES}

\section{Supplementary methods - Antibodies}

\begin{tabular}{|l|l|}
\hline $\begin{array}{l}\text { DYRK1A protein quantification } \\
\text { (Western Blot) }\end{array}$ & mouse anti-FLAG antibody (1:1:000; Sigma Aldrich \#F1804) \\
\cline { 2 - 2 } $\begin{array}{l}\text { DYRK1A localization } \\
\text { (Immunocytochemistry) }\end{array}$ & mouse anti-GFPantibody (in house) \\
\hline Immunoprecipitation & rabbit anti-DYRK1A antibody (1:1000; Cohesion Biosciences \#CPA1357) \\
\hline Interaction with DCAF7 & anti-WDR68 antibody (1:2500; abcam ab138490) \\
\hline DYRK1A autophosphorylation & rabbit anti-phospho-HIPK2 antibody (1:1000, Thermofisher \#PA5-13045) \\
\cline { 2 - 2 } & rabbit anti-DYRK1A antibody (1:1000; Cohesion Biosciences \#CPA1357) \\
\hline MAPT phoshorylation & mouse anti-TAU-5 antibody (Thermofisher \#MA5-12808) \\
\cline { 2 - 2 } & rabbit anti-pTAU-T212 antibody (Thermofisher \#44-740G) \\
\cline { 2 - 2 } & mouse anti-FLAG antibody (1:1:000; Sigma Aldrich \#F1804) \\
\cline { 2 - 2 } & Mouse anti-GAPDH (Sigma \#MAB374) \\
\hline
\end{tabular}

\section{Supplementary text}

\section{Details on splice and frameshift variants and their consequences on DYRK1A mRNA}

Deletions encompassing DYRK1A and chromosomal rearrangement $\mathrm{t}(9 ; 21)(\mathrm{p} 12 ; \mathrm{q} 22)$ interrupting the gene were reported in four individuals (Ind \#1-3, 40). We identified recurrent nonsense variants p.Arg $117 *($ Ind \#4, \#5, \#6), p.Arg205* (Ind \#44), p.Arg255* (Ind \#7, \#43), p.Arg437*(Ind\#34) and p. p.Thr467* (Ind\#42) as well as novel nonsense variants (Ind\#35, \#37) or small indels (Ind \#8-17, \#39, \#41), one of them occurring in the last exon affecting the distal region of the protein: p.Ser660fs (Ind \#18). Seven of the variants identified are predicted to affect splice sites, half of them previously reported elsewhere (Ind \#19-24, \#36, \#38). The remaining individuals (Ind \#25-33) carry missense variants, three of them already reported: p.Gly168Asp, p.Asp287Val and p.Arg467Gln. We tested the consequences of splice variants identified on DYRK1A mRNA (Figure S2) when possible (blood or fibroblasts available) by RNA-sequencing (Ind \#19, 22, 24) or RT-qPCR (Ind \#18). We found that c.328-1G>T (Ind \#19) leads to abnormal splicing events between exons 4 and 5 including intron 4 retention and use of alternative cryptic acceptor sites in exon 5 (Figure S2A). The c.951+4_951+7 del (Ind \#22) leads to retention of intron 7 or skipping of exon 7, both resulting in a premature truncation (Figure S2B). RNA sequencing performed on mRNA extracted from Ind \#24 fibroblasts revealed that the TAA insertion at the beginning of exon 10 was included in the transcripts, leading to a stop codon p.Glu414*. DYRK1A mRNA levels were only slightly decreased in Ind \#22 and \#24 when compared to individual carrying truncating variant (Ind \#11), suggesting that the aberrant transcripts escape nonsense mediated mRNA decay (NMD), at least partially (Figure S2D). The variant previously reported c.1232dup, p.Arg413fs (Bronicki \#10) ${ }^{1}$ is subjected to NMD as illustrated by the decrease of DYRK1A mRNA level in patient's fibroblasts, and the low level of mutant allele in cells, restored by NMD blocking agent (Figure S2E). However, it is worth noting that mutant transcripts carrying the distal frameshift c.1978del (Ind \#18), located in the last exon of the gene, escape to NMD and therefore result in a truncated protein p.Ser660fs having its entire kinase domain (Figure S2F). 


\section{Clinical manifestations in individuals with pathogenic variant in DYRK1A}

All the individuals with clearly loss-of-function variants in DYRK1A present with moderate to severe ID except two (Ind \#3, \#34). Language was affected in all individuals, severely in most (no speech, or only few words or short sentences). Individuals tend to present failure to thrive, even sometimes from the uterine stage (20/32), especially on the weight gain (from -1 to $-4 \mathrm{SD}$ ). The size is less affected comprised between -0.5 to -2SD. Microcephaly is however a constant trait, although not always present from birth. All individuals except two (31/33) had feeding difficulties during the neonatal period (poor sucking, gastrostomy, etc), which can be very severe during infancy and can persist during childhood and even into adulthood (selective, smashed food only, etc). The large majority of individuals present a history of seizures (29/33) mainly including febrile episodes $(n=18)$. Hypotonia was noted in only half of the individuals $(16 / 29)$, but a majority had motor delay with a walk acquired after 18 months of age (27/33)(mean $=23$ months), and the gait could continue to be unstable and ataxic (9). Hypereflexia and hypertonia have been observed in some patients (12). Sleep disorders were reported in some patients (10). Behavioral manifestations observed in patients included anxiety (13) and autistic traits with stereotyped behaviours (24). A diagnosis of autism spectrum disorder (ASD) has been established in only four patients, but few have had the appropriate tests. Other behavioral manifestations such as water fascination (5), absence of fear (5) were noted. MRI revealed ventricular dilation (9), thin corpus callosum (9), as well as cortical or cerebellar atrophy (9). Other manifestations include gastrointestinal manifestations such as constipation (14) or gastroesophageal reflux (10), intestinal anomalies such as inguinal hernia (4) and urogenital anomalies already reported to be frequent ${ }^{2}$, including cryptoorchidism (4). No obvious kidney anomalies have been reported but not all the individuals underwent a renal ultrasound. We observed a thin skin in a high frequency of patients (15), often associated with dermatitis or atopic skin after birth or during infancy (11), sometimes very pronounced. After reanalysis of literature, we found that atopic demartitis was also reported in additional individuals. Vision anomalies include myopia (6), hypermetropia (8) and astigmatism (6). An optic nerve hypoplasia was noticed in at least three individuals. A papillary pallor was reported in some individuals (6). Individuals shared common facial appearance including erratic hairline with thin hair, deep set eyes with upper eyelid edema, protruding nose or pointed nasal tip, dysplastic ears, thin upper lip, widely spaced teeth with protruding upper dental arch, retro/micrognathism (Figure 1, Figure S3).

\section{Effect of missense variants on DYRK1A subcellular localization}

The cellular localization of overexpressed WT and variant DYRK1A proteins in Hela cells, studied by immunostaining, revealed three types of cellular distributions: 1) mainly nuclear (N), 2) nuclear and cytoplasmic (N+C) and 3) mainly cytoplasmic (C) (Figure S8C). WT DYRK1A protein is mainly localized in the nucleus $(80 \% \mathrm{~N} ; 20 \% \mathrm{~N}+\mathrm{C})$, and this localization is affected when we mutated, separately or combined, the two nuclear localization signals NLS1 (aa 117-134) and NLS2 (aa 389-395) confirming that each NLS contributes to the nuclear localization of DYRK1A, as previously reported ${ }^{3}$. We observed a significant decrease of nuclear localization for Arg413fs, but none of the missense variants tested seems to affect DYRK1A localization. 


\section{Generating and phenotyping analysis of the DYRK1A Thr588Asn Mutant mouse line}

The Dyrk $1 a^{T 588 N}$ mutant mouse line was established for YH at the Institut Clinique de la Souris-(PHENOMINICS, Illkirch, France; http://www.phenomin.fr) and breed in our animal facility at the PHENOMIN-ICS (Agreement C67-218-40). The targeting vector was constructed as follows. A $3.4 \mathrm{~kb}$ fragment encompassing part of intron 11 and corresponding to the 5' homology arm was amplified by PCR on C57BL/6N ES cell genomic DNA and subcloned in an MCI proprietary vector containing two multiple cloning sites, three repeated SV40 polyA sequences as well as a flipped Neomycin resistance cassette surrounded by 2 LoxP sites. The variant (ACC > A $\underline{A}$ C) leading to the threonine to asparagine change at position 588 (T588N) was introduced in a second cloning step by fusion of 2 PCR products. Finally, a $3.6 \mathrm{~kb}$ fragment corresponding to the 3 ' homology was cloned in a third cloning step to obtain the final targeting construct. The linearized construct was electroporated in C57BL/6N mouse embryonic stem (ES) cells (ICS proprietary line S3). After G418 selection, targeted clones were identified by long-range PCR and further confirmed by Southern blot with an internal (Neo) probe and a 3' external probe. Two positive ES clones were validated by karyotype spreading and microinjected into BALB/cN blastocysts. Resulting male chimeras were bred with Flp deleter females showing maternal contribution ${ }^{4}$. Germline transmission with the direct excision of the selection cassette was achieved in the first litter. The mouse line was bred on a pure C57BL/6N genetic background in a specific pathogen free environment at the PHENOMIN-ICS animal mouse, with poplar wood granulate bedding (SAFE, Augis, France) and access to normal diet (D03 and D04; SAFE, Augis, France) and tap water treated with Chlorine dioxide (0,8ppm) ad libitum under a classical 12-12 light dark cycle (dark 7pm to $7 \mathrm{am}$ during phenotyping). We found a close to mendelian transmission ratio for the segregration of the variant both in heterozygotes and homozygotes. The female to male ratio was as expected.

The expression of the Thr588Asn allele of Dyrkla in heterozygous was studied by Western blot at 7 weeks of age in 5 wild type and 5 Dyrkl $a^{T 588 N /+}$ animals. Twenty micrograms of proteins per sample were separated by classical electrophoresis and transferred on a membrane (BIO-RAD, Schiltigheim, Fr). Incubation with primary antibody Anti-Dyrk1a (Abnova, 1/1000) was followed with an incubation with a secondary antibody Anti-mouse (Abnova; 1/5000). We used B-Actin as an internal control that was detected with an anti-mouse B-actin-HRP (Sigma, 1/150000). For revelation, we used the Clarity ${ }^{\text {TM }}$ Western ECL Substrate (BIO-RAD\#170-5061). No statistically meaningful difference was observed between the genotypes (Student t.test=0.33; Figure S12B). We performed a functional characterization of DYRK1A kinase activity following the protocol

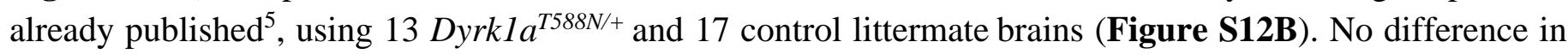
DYRK1A kinase activity was found regarding the sex or genotype. We raised a cohort with mutant and control

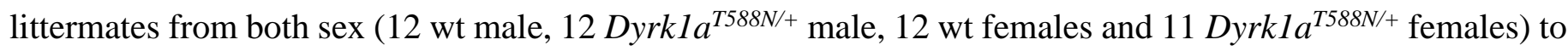
test the cognition, memory, locomotor activity, and assess the anxiety and autism like stereotypies on these animals. We performed the battery of tests as shown in (Figure S12D) following the pipeline and protocols previously described ${ }^{6-9}$. All the animal experiments were done in compliance with the ARRIVE Guidelines 10,11 in accordance with the Directive of the European Parliament: 2010/63/EU, revising/replacing Directive 86/609/EEC and with French Law (Decret n ${ }^{\circ}$ 2013-118 01 and its supporting annexes entered into legislation 01February 2013) relative to the protection of animals used in scientific experimentation and supervised by the Com'eth our local ethical committee. The tests were administered in the following order: open field, novel object recognition (noted NOR, performed $24 \mathrm{~h}$ after the open field), Y-maze, repetitive behaviour, sociability 3 chambers test and reciprocal tests. In the open field test we assessed the locomotor activity, exploratory drive and anxiety; and we have taken into account randomization of the animals, blinding of the experimenter during the animal research. With the EthoVision system (Noldus, the Netherlands), we measured the total distance, time spend on each of the three areas centre, periphery and walls. Sex was not affecting any of these 
parameters, so the downstream statistical assessments was done pooling all the animals together divided just by genotype and not considering the sex, increasing the sampling size. We found no difference or effect between the control and mutant Dyrk1 $a^{T 588 N /+}$ mice in the total distance travelled or time spend on each area. All the animals performed the test and did not show anxiety as they spent quite a high amount of time travelling over all the arena. The novel object recognition test (NOR) was used to study the memory of the animals to discriminate and explore novel objects over familiar ones. We did not identify a deficit in object recognition between the two genotypes (Figure S12E). We evaluated the motor activity and the working memory in the Y maze. There was no difference in the percentage of visits done to each arm, no defect in the spontaneous alternation (Figure S12F) or delay leaving the initial arm. Repetitive behaviour is one of the 3 main clinical manifestations of autism, together with deficits in social interaction and communication. Thus, we analysed in a 10 min test the number and time spend climbing, digging, and rearing by the mutant animals and control littermates but we did not observe any special stereotypic of repetitive behaviour in the Dyrkla $a^{T 588 N /+}$ mice except a slight increase in climbing frequency (Figure S12G). In the three chambers test, we analysed both the time spent in proximity of the empty cage during the presentation, or for the familiar and then the new congener in the discrimination phase. No phenotype was detected in the presentation phase and a significant difference in exploration was observed for the Thr588Asn mutant in the discrimination phase (Figure S12H). For the reciprocal sociability test where the social interactions between pairs of mice is analyzed, always using congeners that were not cage mates and in the case of both mutants and control mice in the cohort adding an unknown wild-type animal of the same sex and similar age and size to assess the interactions. We analysed both the time spend in proximity nose to nose or proximity nose to tail but no difference in interaction was observed for Dyrk1 $a^{T 588 N /+}$. Although is worthy to mention that the Gardner-Altman effect size plots show a slight tendency on the Dyrk $1 a^{T 588 N /+}$ mice to decrease the number of contacts and increase the distance with the congener (Figure S12I).

\section{Supplementary acknowledgements}

We would like to thank the Centre National de Génotypage for their participation in library preparation and DNA sequencing. We thank all the people from the GenomEast sequencing platform, IGBMC cloning platform for their technical and bioinformatics supports. They thank people for the diagnostic laboratory of Hôpitaux Universitaire de Strasbourg (HUS) for performing follow-up of variants and giving diagnosis to family as well as additional clinicians not included in the author list for their clinical contributions.

\section{Supplementary Figures}

\section{Figure S1. Variants identified in DYRK1A in individuals with ID}

Schematic representation of DYRK1A protein secondary structure with its different domains: Nuclear localization sequence 1 and 2 (NLS1 and NLS2); DYRK Homology box (DH); catalytic domain; PEST domain (PEST); His rich domain (His) and Serine Threonine (S/T) repeat domain. Variants identified in the cohort are represented with the number of individuals (Ind\#) carrying then (A) truncating variants (nonsense, frameshift, splice variants) (B) missense variants. The variants identified tested in this study are indicated in gold. 


\section{Figure S2. Consequences of variants on DYRK1A mRNA}

Sashimi plot from Integrative Genome Viewer (IGV) showing consequences of the splice variants identified by RNA-Seq in mRNA extracted from (A) Ind \#19 blood, showing that c.328-1G>T generate different abnormal transcripts with a) intron 4 retention, leading to a premature stop codon (p.Tyr111Argfs*6), b) use of an alternative acceptor site $18 \mathrm{bps}$ or c) $21 \mathrm{pbs}$ downstream the regular one, leading to deletion of few amino acids (p.Val110_Lys115del or p.Val110Lys116del) (B) Ind \#22 fibroblasts, showing that c.951+4_951+7del variant leads to a skipping of exon 7 in half of the mutated transcripts (p.Val222Aspfs*22) and a retention of intron 7 in the other half (p.Ile318fs*10); (C) IGV view of RNA-seq obtained from Ind \#24 fibroblasts, showing c.1240-1_1240insTAA causes the insertion of these 3 nucleotides in the mRNA at the beginning of exon 10 leading to a premature stop codon p.(Glu414*) (D) Quantitative expression of DYRK1A mRNA (normalized by the expression of two reference genes, GAPDH and YWHAZ) (E) Sequencing of DYRK1A mRNA in Individual Bronicki\#10 cells (c.1232dup, p.(Arg413fs)) treated or not with a NMD blocking agent (emetine). Sequencing of blood DYRK1A cDNA in (F) Ind \#18 showing an equal amount of transcripts carrying the c.1978del variant compared to wild-type allele, suggesting that mutated transcripts escape to $\mathrm{NMD}$, and in (G) Ind \#30, carrying the variant c.1098G>T, showing the skipping of exon 8 induced by this variant r.952_1098del) leading to the deletion of 49 amino acids p.Ile318_Glu366del instead of one amino acid substitution p.Glu366Asp as first predicted (the probability of using exon 8 donor splice site was decreased by the variant: MaxEnt: $-72.7 \%$; NNSPLICE: $-54.1 \%$ ).

\section{Figure S3. Photographs of individuals with variant in DYRK1A}

Photographs of individuals with variants in DYRK1A, showing that they share similar facial features.

\section{Figure S4. Distribution of the clinical scores calculated without photograph (on 15 points)}

Clinical scores calculated without photograph for individuals carrying pathogenic variants in DYRK1A from the initial cohort (DYRK1A_I), the replication cohort (DYRK1A_R) and the individuals affected with other frequent monogenic forms of ID, associated to pathogenic variants in ANKRD11, MED13L, DDX3X, ARIDIB, SHANK3, TCF4 or KMT2A. Brown-Forsythe and Welsh ANOVA tests with Dunnett's T 3 multiple comparisons test were performed. ns: not significant; ** $\mathrm{p}<0.01$; $* * * \mathrm{p}<0.001$, error bars represent SD.

\section{Figure S5. Predictions of effect of missense variants and conservation of DYRK1A protein}

(A) Distribution of the CADD score for missense variants a) not presumed to be not disease-causing (negative set, $\mathrm{N}$-set, $\mathrm{n}=115$, see Methods), b) presumed to be pathogenic (positive set, $\mathrm{P}$-set, $\mathrm{n}=16$ ) and c) other missense variants (test set, $\mathrm{T}$-set, $\mathrm{n}=41$ ). A CADD score $>=20$ means that the variant belongs to the top $1 \%$ of variants predicted to be the most deleterious, $>=25$ that the variant belongs to the top $0.3 \%$ of variants predicted the most deleterious $>=30$ the variant belongs to the top $0.1 \%$ of variants predicted the most deleterious. BrownForsythe and Welsh ANOVA tests with Dunnett's T 3 multiple comparisons test were performed. ns: not significant; ** $\mathrm{p}<0.01$; $* * *<\mathrm{p}<0.001$, error bars represent SD (B) Percentage of variants from the $\mathrm{N}$-set (presumably benign), P-set (presumably pathogenic) and T-set (to test) having a CADD score $>=20,>=25$ 
or $>=30$, or conserved across $100 \%$ of Vertebrate species $(\mathrm{V}=100 \%)$, conserved in at least $90 \%$ of Metazoan species $(\mathrm{M}>=90 \%)$, and at least $80 \%$ of other animals $(\mathrm{O}>=80 \%) .{ }^{\mathrm{a}} \mathrm{p}$.Arg158His variant $(\mathrm{V}=100 \% ; \mathrm{M}=96 \%$; $\mathrm{O}=92 \%) ;{ }^{\mathrm{b}}$ all variants except p.Ser311Phe variant $(100 \% ; 100 \% ; 71 \%)$, p.Thr588Asn $(\mathrm{V}=85 \%)$ and p.Ala277Pro (100\%; 12\%; 27\%); variants ; ${ }^{c}$ p.Gly171Arg (100\%; 100\% ; 86\%), p.Lys188Arg (100\%; 96\%; 97\%), p.Leu207Pro (100\%; 93\% ; 93\%), p.Leu241Pro (100\%; 100\%; 97\%), p.Leu245Arg (100\%; 100\%; 97\%), p.Asp287Tyr (100\%; 100\%; 97\%), p.Asp287Asn (100\%; 100\%; 97\%), p.Pro290Arg (100\%; 100\%; 97\%), p.Arg328Trp (100\%; 100\%; 95\%), p.Leu347Arg (100\% ; 96\% ; 84\%).

\section{Figure S6. Conservation of DYRK1A proteins across the different taxons}

Schematic view of the Multiple Sequence Alignment (MSA) of DYRK1A protein orthologs from Vertebrates, Metazoans, Protists, Fungi and Plants. MSA positions are numbered according to the human protein and colored by a red gradient according to the level of amino acid conservation in each group.

\section{Figure S7. Effect of variants on DYRK1A half-life and interaction with DCAF7}

Investigation of variants consequences on DYRK1A protein stability and interaction with its partner DCAF7. (A) Stability of DYRK1A proteins in HEK293 transfected with DYRK1A plasmids and treated with cycloheximide $40 \mu \mathrm{g} / \mathrm{mL}$ (stopped at 1, 2, 4 and 8 hours after treatment). Constructs was detected by SDSPAGE by immunoblotting whole cell lyzates using an anti-FLAG antibody and quantification realized with GAPDH (B) Co-immunoprecipitation of DYRK1A in HEK293 cells transfected with DYRK1A plasmids using an anti-FLAG antibody, without antibody (Empty) or with Mouse against Rabbit antibody (MAR) as negative and species isotype controls. DCAF7 (WDR68) interaction was detected by SDS-page immunoblotting with a specific antibody (1:2500; abcam anti-WDR68 antibody ab138490).

\section{Figure S8. In vitro effects of additional variants on DYRK1A proteins and cellular localization of variant proteins}

(A-B) Effect of additional variants on DYRK1A level and ability to autophosphorylate: one variant from the $\mathrm{N}$-set (reported twice in gnomAD) but affecting an highly conserved amino acid position, Arg158His, one variant from the P-set but affecting a position poorly conserved after vertebrates, Ala277Pro, and three variants initially reported as VUS in ClinVar (the last one was reclassified as Likely Pathogenic during the course of this study) and affecting highly conserved positions : p.Gly171Arg, p.Leu241Pro and p.Pro290Arg. We also tested the functional effet of two nonsense changes we created for the need of this study: Ser660* and Ser661*. (A) Level of variant DYRK1A proteins expressed in HEK293 ( $\mathrm{n}=3$ series) cells transiently transfected with DYRK1A constructs. Protein levels were normalized on the level of GFP proteins (expressed from a cotransfected pEGFP plasmid). One-way ANOVA with multiple comparison test was performed to compare the level of variant DYRK1A proteins to the level of wild-type DYRK1A protein (orange dashes), applying Bonferroni's correction: ns: not significant; ${ }^{*} \mathrm{p}<0.05 ;{ }^{*} \mathrm{p}<0.01 ; * * \mathrm{p}<0.001$; error bars represent SEM, standard error of the mean, (B) DYRK1A's ability to autophosphorylate on Tyr321 was tested in HEK293 cells $(n=3)$ by immunoprecipitations with anti-DYRK1A followed by an immunoblot using an antiphospho-HIPK2 as described in Widowati et al. DYRK1A phospho-Tyr321 levels were normalized with DYRK1A total DYRK1A protein levels (orange dashes), and expressed as percentage of WT level. One-way ANOVA test was performed to compare variants to wild-type DYRK1A levels. ns: not significant; 
$* * * \mathrm{p}<0.001$; error bars represent SEM, standard error of the mean $(\mathbf{C})$ Cellular localization of DYRK1A variant proteins observed in HeLa cells after overexpression of FLAG-tagged wild-type and variant DYRK1A constructs ( $\mathrm{n}=3$ series of Hela cells; 50 cells minimum counted per series ; scale bars of illustrative images correspond to $10 \mu \mathrm{m})$. Three types of localization of DYRK1A protein in cells have been observed: DYRK1A located mostly in the nucleus $(\mathrm{N})$, in both nucleus and cytoplasm $(\mathrm{N}+\mathrm{C})$, or mostly in the cytoplasm $(\mathrm{C})$. Scale bars: $50 \mu \mathrm{m}$. WT protein is mainly localized in the nucleus (N/N+C/C: $80 / 20 / 0 \%)$. The shift into cytoplasmic localization of DYRK1A is stronger when NLS1 is disrupted (0/63/37\%) compared to NLS2 (22/71/7\%), and even more drastic when both are mutated, with DYRK1A stacked in the cytoplasm in a large majority of cells $(0 / 26 / 74 \%)$. Chi-square test was performed to compare localization of variant DYRK1A proteins to wildtype DYRK1A protein ns: not significant; *** $<<0.001$; error bars represent SEM, standard error of the mean. (D) Immunofluorescence experiment showing that the Ser661* variant does not lead to DYRK1A protein aggregation when overexpressed in HeLa cells.

\section{Figure S9. DNAm profile of all DYRK1A cases DYRK1A DNAm signature sites.}

Heatmap showing the hierarchical clustering of discovery DYRK1A LoF cases ( $\mathrm{n}=10$; grey) and age- and sexmatched neurotypical discovery controls $(n=24$; blue) used to identify the 402 differentially methylated (DM) signature sites shown (each row corresponds to a $\mathrm{CpG}$ site). The DNAm values for DYRK1A LoF validation cases $(n=6$, red), missense variants $(n=10$, yellow) and distal LoF variant $(n=1$, green) are shown. The colour gradient represents the normalized DNA methylation value from -2.0 (blue) to 2.0 (yellow) at each site. Ind \#33 (Gly486Asp) has an opposite DNAm values to other DYRK1A cases at these sites, also clustering out from controls. Euclidean distance metric is use for the clustering dendrogram.

\section{Figure S10. Effect of variants on DYRK1A kinase activity regarding MAPT (TAU) Thr212 phosphorylation levels.}

MAPT Thr212 phosphorylation assays performed to test variants with particular profiles : Ser324Arg (partial autophosphorylation), Gly486Asp (potential GoF), Thr588Asn (potential phenocopy), Ser660fs (protein aggregates) as well as Ser660* and Ser661*. Immunoblots on total protein extracts from HEK293 cells transfected with DYRK1A and MAPT plasmids using anti-FLAG, anti TAU-5 and anti-pT212-TAU antibodies (A) Transfection of different quantities of DYRK1A WT plasmid and $1 \mu \mathrm{g}$ of MAPT plasmid (B) Transfection of DYRK1A proteins harboring the distal truncating variant Ser660fs or the two nonsense changes Ser660* and Ser661* and $1 \mu \mathrm{g}$ of MAPT plasmid (C) Transfection of other DYRK1A variants : Ser311Phe (known to abolish kinase activity), Ser324Arg, Gly486Asp and Thr588Asn and $1 \mu$ g of MAPT plasmid.

\section{Figure S11. DNAm profile of Ind \#33 with DYRK1A Gly486Asp variant displays an opposite DNAm profile at individual signature CpGs.}

DNA methylation values are shown at the 25 most hypermethylated and hypomethylated CpG sites in the signature. Red bars show the average delta beta value at each site in DYRK1A LoF discovery cases $(\mathrm{n}=10)$, error bar represents the standard error of the mean (SEM). Yellow bars represent the delta beta value Ind \#33 
at these sites (the beta value of Ind \#33 minus the average beta of discovery controls). At most sites, the delta beta for Ind \#33 is opposite that of DYRK1A cases, indicating DNAm levels in the opposite direction of DYRK1A LoF cases relative to controls. Importantly, this is a different phenomenon than Ind \#33 having control-like DNAm values, in which case the bars would be near 0.

Figure S12. Generation, characterisation and behavioural analysis of the heterozygous mouse mutant line carrying a Thr588Asn variant in Dyrk1a. (A) Generating the Thr588Asn (T588N) allele. The T588N allele phenotyped is named 'T588N with a WT potential allele' and was obtained by homologous recombination in embryonic stem cells using a targeting vector. LoxP and Flp sites are indicated respectively in green and grey (B) No difference in western blot quantification of DYRK1A level, normalized to b-actin level, in 5 hippocampal protein extracts isolated from Dyrk $1 a^{T 588 N /+}$ individuals and 5 control littermates (C) Simarly no changes was observed in the phosphorylation of a specific DYRK1A-phosphorylation peptide (tyde) using brain extract isolated from the hippocampi of Dyrkla ${ }^{T 588 N /+}$ and control littermates (respectively $\mathrm{n}=13$ and 17) (D) schematic representation of the behavioural pipeline used to study 23 Dyrk1a ${ }^{T 588 N /+}$ and 24 wild-type (wt) littermates from both sexes at the starting age of 10 weeks (E) In the novel object recognition, a preference was found in the percentage of time exploring the novel object (NO) compared to the familiar object (FO) in the Dyrkla $a^{T 588 N /+}$ (F) Spontaneous alternation in the Y maze was not affected by the variant (one single $\mathrm{t}$ test with $50 \%$ chance level). (G) A significant difference was observed in specific repetitive behaviour climbing, but not in digging or rearing activities analysed during the repetitive behavioral test $(\mathbf{H})$ In the three-chamber sociability test, the percentage of time exploring the familiar stranger (F) versus the novel stranger $(\mathrm{N})$ was significantly different in the mutant $\mathrm{T} 588 \mathrm{~N}$ heterozygotes (I) During freely moving social interaction we did not detect any anomaly. The statistical significance is noted as followed *adj P.value $=<0.05, * * 0.05<$ adj P.value $<0.01, * * *$ adj P.value $<0.001$

\section{Table S1. Clinical information and summary of recurrent clinical signs observed in individuals with pathogenic variants in DYRK1A}

M: male; F: female; IUGR: Intrauterine growth restriction; CMV: research for cytomegalovirus infection; BW: birth weight; BS: birth size; BOFC: birth occipitofrontal head circumference; A/FW: absence/few words; ID: intellectual disability, ASD: Autism spectrum disorder; M/S: moderate/severe; mo; months old; SD: standard deviation; AtSD: atrial septal defect; VSD: ventricular septal defect; GER: Gastroesophageal reflux; MRI: Magnetic resonance imaging; EV: enlarged ventricles, CCA/H: corpus callosum agenesis/hypoplasia, CA: cortical or subcortical atrophy, CeA: cerebellar atrophy; NA: not available; ${ }^{a}$ Previously reported: Individuals with truncating variants in DYRK1A reported in literature $(\mathrm{n}=80)^{1,2,12-36}$

\section{Table S2. Calculation of the clinical score CS $_{\text {DYRK1A }}$ in the different cohorts}

NA: not available; DYRK1A_I: Initial cohort of individuals with variants disrupting DYRK1A (nonsense, frameshift, splice, deletions or translocations) reported in this study, used to set up the clinical score ; DYRK1A_R: Cohort of replication including individuals with variants disrupting DYRK1A who were previously described with enough clinical information and photographs available $(n=12)^{1,12,18} *$ mild macrocephaly 
Table S3. List of DYRK1A missense variants reported in databases (gnomAD, ClinVar, Decipher) and literature and identified in this report (N-set, P-set and T-set)

training sets: N-set: variants not presumed to be disease-causing, i.e missense variants annotated as "benign"/“likely benign" in ClinVar as well as variants reported more than once in GnomAD (november 2019 release) ( $n=115)$; P-set: missense variants reported as "pathogenic"/“likely pathogenic" in Clinvar ( $\mathrm{n}=16) \mathrm{T}$ set: missense variants reported here, in literature, or as VUS in Clinvar $(n=44)$; FreqRefV, FreqRefM, FreqRefO, FreqRefP, FreqRefF: frequency of the reference amino acid among Vertebrates, Metazoans, Other protist animals, Plants or Fungi; FreqSubV, FreqSubM, FreqSubO, FreqSubP, FreqSubF : frequency of the novel amino acid among Vertebrates, Metazoans, Other animals, Plants or Fungi;

\section{Table S4. List of CpG sites included in DYRK1A DNAm signature}

GoF: gain-of-function. One third of the signature sites (134/402) the $\beta$ value for p.Gly486Asp was outside the range observed for that of all discovery controls.

\section{Table S5. SVM score associated with the different variants}

SVM: Support Vector Machine classification and scores

\section{Table S6. Summary of the analysis performed to reclassify variants in DYRK1A}

${ }^{1}$ Highly conserved : $\mathrm{V}=100 \% \mathrm{M}>=90 \% \mathrm{O}>=80 \% *$ The consequences of $\mathrm{c} .1098 \mathrm{G}>\mathrm{T}$ is p.Ile318_Glu366del instead of p.Glu366Asp; $* *$ same individual submitted twice $* * *:$ this amino acid change is not reported in gnomAD, but Thr588Pro is reported 44 times (including in one homozygote) and Thr588Ala is reported once ; DD: developmental delay, ID: intellectual disability ; clinical information reported in ClinVar and/or in littérature for the individual a: "Abnormality of the frontal hairline, abnormality of the skin, cataract, cerebellar atrophy, feeding difficulties in infancy, intrauterine growth retardation, microcephaly, proportionate short stature, single transverse palmar crease, specific learning disability, ventriculomegaly"; b: «Short chin, Truncal obesity »; ${ }^{c}$ : «Developmental regression, intellectual disability, hypotonia, mild ataxia, intention tremor, dysmorphisms, primary microcephaly, failure to thrive, demyelination, sun sensitivity, incontinence and anxiety »; ${ }^{\mathrm{d}}$ : «ASD, learning disorder and macrocephaly »; ${ }^{\mathrm{e}}$ : « Abnormal facial shape, Down-sloping shoulders, Genu valgum, Global developmental delay, Hypoplastic toenails » in an individual carrying an additional nonssense variant in DYRK1A; ${ }^{\text {: } ~ « D e c r e a s e d ~ f a c i a l ~ e x p r e s s i o n, ~ G l o b a l ~ d e v e l o p m e n t a l ~ d e l a y, ~}$

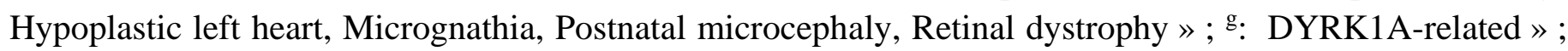
h: «MR/ID/DD; Seizures; Brain MRI positive, Dysmorphic features, Cardiovascular; Craniofacial; Hematologic (child onset), Gastrointestinal (child onset); Musculoskeletal/Structural; Neurologic (child onset), Ophthalmologic, Renal conditions »; ${ }^{\mathrm{i}}$ : «Brachycephaly, Intellectual disability, Seizures, Muscular hypotonia, Global developmental delay »; dn : de novo; ACMG/AMP Criteria : PS2 : De novo confirmed ; PS3 : in vitro or in vivo functional studies supportive of a damaging effect on the gene or gene product; PM2 :Absent from controls ; in Exome Sequencing Project, 1000 Genomes or ExAC ; PP3 : in silico analysis support a deleterious effect on the gene product ; PP4 :Patient's phenotype is highly specific for gene ; BS1: Allele frequency is greater than expected for disorder; BS3 :Well-established in vitro or in vivo functional studies shows no damaging effect on protein function or splicing ; BP4 :in silico analysis suggest no impact on gene or gene product. 
Table S7. Summary of functional studies previously performed for additional missense variants in DYRK1A

${ }^{1}$ Highly conserved : $\mathrm{V}=100 \% \mathrm{M}>=90 \% \mathrm{O}>=80 \% *$ The consequences of $\mathrm{c} .1098 \mathrm{G}>\mathrm{T}$ is p.Ile318_Glu366del instead of p.Glu366Asp ; DD: developmental delay, ID: intellectual disability ; Clinical information reported in ClinVar and/or in litterature : a: "speech delay, motor delay, moto coordination disorder, seizures and mild physical dysmorphy"; b: "mild ID, infantile spasms, speech delay, social interaction and repetitive behaviors, anxiety"; c: "Abnormality of the palmar creases, abnormality of the skeletal system, amblyopia, astigmatism, cleft soft palate, constipation, delayed speech and language development, global developmental delay, intrauterine growth retardation, microcephaly"; d: "IUGR, DD, microcephaly, ID, severe speech delay"; ": "ASD+ID, anxiety, perseveration and additional upsets and aggressive behavior"; f. "Severe ID, seizures febrile + generalized tonico-clonic"; g: "ID, seizures and microcephaly"; h: "Intellectual disability, microcephaly, feeding difficulties; absent or delayed speech development; seizures; deeply set eye"; i. "Global developmental delay, Microcephaly, Seizures"; j: "Microcephaly, ID, seizures, spasticity, global developmental delay, motor delay, hypertonia, abnormal facial shape, cortical dysplasia, short stature, cortical gyral simplification"; dn : de novo ; ACMG/AMP Criteria : PS2 : De novo confirmed ; PS3 : in vitro or in vivo functional studies supportive of a damaging effect on the gene or gene product; PM2 :Absent from controls; in Exome Sequencing Project, 1000 Genomes or ExAC; PP3 : in silico analysis support a deleterious effect on the gene product; PP4 :Patient's phenotype is highly specific for gene ; BS1: Allele frequency is greater than expected for disorder ; BS3 :Well-established in vitro or in vivo functional studies shows no damaging effect on protein function or splicing ; BP4 :in silico analysis suggest no impact on gene or gene product.

\section{Table S8. Distal frameshift variants (last exon) identified in individuals with NDD}

Three distal frameshift variants are reported in Clinvar and in literature ${ }^{16}$. Their clinical interpretation remains ambiguous, especially for c.1726C > T p.Gln576* and c.2040C >A p.Tyr680*, for which inheritance is unknown and clinical manifestations do not really overlap those of DYRK1A syndrome. "a: "Severe psychomotor delay, no walk aquisition and no language, severe amblyopia, self-injurious behavior, ASD, dysmorphic features (frontal bossing, hypertelorism, nystagmus, epicanthal folds, a flat nasal bridge, bilateral low-set ears, down-slanting palpebral fissures, a short philtrum, a high arched palate, downturned mouth and micrognathia). Relative macrocephaly (OFC: $52 \mathrm{~cm},+0.6 \mathrm{SD}$; weight: $14.6 \mathrm{~kg},-2.2 \mathrm{SD}$; height: $103.5 \mathrm{~cm}$, -3.1 SD)" 16; b: "Infantile spasms, west syndrome, ASD, aggression, hyperactivity" (personal communication Aida Telegrafi, Genedx); However, the most distal variant ever reported in DYRK1A, c.2213_2218delinsAGAG p.Thr738fs, occurred de novo in an individual with clinical features consistent with DYRK1A syndrome. ": "Autism, microcephaly, seizures, history of failure to thrive and IUGR, mitochondrial complex IV deficiency noted on muscle biopsy" (personal communication Aida Telegrafi, Genedx).

\section{References}

1. Widowati EW, Ernst S, Hausmann R, Müller-Newen G, Becker W. Functional characterization of DYRK1A missense variants associated with a syndromic form of intellectual deficiency and autism. Biol Open. 2018;7(4). doi:10.1242/bio.032862 
2. Quartier A, Courraud J, Thi Ha T, et al. Novel mutations in NLGN3 causing autism spectrum disorder and cognitive impairment. Hum Mutat. 2019;40(11):2021-2032. doi:10.1002/humu.23836

3. Mattioli F, Isidor B, Abdul-Rahman O, et al. Clinical and functional characterization of recurrent missense variants implicated in THOC6-related intellectual disability. Hum Mol Genet. 2019;28(6):952-960. doi:10.1093/hmg/ddy391

4. Lee K-S, Choi M, Kwon D-W, et al. A novel de novo heterozygous DYRK1A mutation causes complete loss of DYRK1A function and developmental delay. Sci Rep. 2020;10(1):9849. doi:10.1038/s41598-020-66750-y

5. Bronicki LM, Redin C, Drunat $S$, et al. Ten new cases further delineate the syndromic intellectual disability phenotype caused by mutations in DYRK1A. Eur J Hum Genet. 2015;23(11):1482-1487. doi:10.1038/ejhg.2015.29

6. Blackburn ATM, Bekheirnia N, Uma VC, et al. DYRK1A-related intellectual disability: a syndrome associated with congenital anomalies of the kidney and urinary tract. Genet Med. 2019;21(12):27552764. doi:10.1038/s41436-019-0576-0

7. Alvarez M, Estivill X, de la Luna S. DYRK1A accumulates in splicing speckles through a novel targeting signal and induces speckle disassembly. J Cell Sci. 2003;116(Pt 15):3099-3107. doi: $10.1242 /$ jcs.00618

8. Birling M-C, Dierich A, Jacquot S, Hérault Y, Pavlovic G. Highly-efficient, fluorescent, locus directed cre and FlpO deleter mice on a pure C57BL/6N genetic background. Genesis. 2012;50(6):482-489. doi:10.1002/dvg.20826

9. Nguyen TL, Duchon A, Manousopoulou A, et al. Correction of cognitive deficits in mouse models of Down syndrome by a pharmacological inhibitor of DYRK1A. Dis Model Mech. 2018;11(9). doi:10.1242/dmm.035634

10. Ung DC, Iacono G, Méziane H, et al. Ptchd1 deficiency induces excitatory synaptic and cognitive dysfunctions in mouse. Mol Psychiatry. 2018;23(5):1356-1367. doi:10.1038/mp.2017.39

11. Marechal D, Lopes Pereira P, Duchon A, Herault Y. Dosage of the Abcg1-U2af1 region modifies locomotor and cognitive deficits observed in the Tc1 mouse model of Down syndrome. PLoS ONE. 2015;10(2):e0115302. doi:10.1371/journal.pone.0115302

12. Arbogast $\mathrm{T}$, Iacono $\mathrm{G}$, Chevalier $\mathrm{C}$, et al. Mouse models of $17 \mathrm{q} 21.31$ microdeletion and microduplication syndromes highlight the importance of Kansl1 for cognition. PLoS Genet. 2017;13(7):e1006886. doi:10.1371/journal.pgen.1006886

13. Dubos A, Meziane H, Iacono G, et al. A new mouse model of ARX dup24 recapitulates the patients' behavioral and fine motor alterations. Hum Mol Genet. 2018;27(12):2138-2153. doi: $10.1093 / \mathrm{hmg} / \mathrm{ddy} 122$

14. Karp NA, Meehan TF, Morgan H, et al. Applying the ARRIVE Guidelines to an In Vivo Database. PLoS Biol. 2015;13(5):e1002151. doi:10.1371/journal.pbio.1002151 
15. Kilkenny C, Browne WJ, Cuthill IC, Emerson M, Altman DG. Improving bioscience research reporting: the ARRIVE guidelines for reporting animal research. PLoS Biol. 2010;8(6):e1000412. doi:10.1371/journal.pbio.1000412

16. van Bon BWM, Hoischen A, Hehir-Kwa J, et al. Intragenic deletion in DYRK1A leads to mental retardation and primary microcephaly. Clin Genet. 2011;79(3):296-299. doi:10.1111/j.13990004.2010.01544.x

17. van Bon BWM, Coe BP, Bernier R, et al. Disruptive de novo mutations of DYRK1A lead to a syndromic form of autism and ID. Mol Psychiatry. 2016;21(1):126-132. doi:10.1038/mp.2015.5

18. O’Roak BJ, Vives L, Fu W, et al. Multiplex targeted sequencing identifies recurrently mutated genes in autism spectrum disorders. Science. 2012;338(6114):1619-1622. doi:10.1126/science.1227764

19. Courcet J-B, Faivre L, Malzac P, et al. The DYRK1A gene is a cause of syndromic intellectual disability with severe microcephaly and epilepsy. J Med Genet. 2012;49(12):731-736. doi:10.1136/jmedgenet-2012-101251

20. Okamoto N, Miya F, Tsunoda T, et al. Targeted next-generation sequencing in the diagnosis of neurodevelopmental disorders. Clin Genet. 2015;88(3):288-292. doi:10.1111/cge.12492

21. Iglesias A, Anyane-Yeboa K, Wynn J, et al. The usefulness of whole-exome sequencing in routine clinical practice. Genet Med. 2014;16(12):922-931. doi:10.1038/gim.2014.58

22. Ruaud L, Mignot C, Guët A, et al. DYRK1A mutations in two unrelated patients. Eur J Med Genet. 2015;58(3):168-174. doi:10.1016/j.ejmg.2014.12.014

23. Ji J, Lee H, Argiropoulos B, et al. DYRK1A haploinsufficiency causes a new recognizable syndrome with microcephaly, intellectual disability, speech impairment, and distinct facies. Eur J Hum Genet. 2015;23(11):1473-1481. doi:10.1038/ejhg.2015.71

24. Rump P, Jazayeri O, van Dijk-Bos KK, et al. Whole-exome sequencing is a powerful approach for establishing the etiological diagnosis in patients with intellectual disability and microcephaly. BMC Med Genomics. 2016;9:7. doi:10.1186/s12920-016-0167-8

25. Luco SM, Pohl D, Sell E, Wagner JD, Dyment DA, Daoud H. Case report of novel DYRK1A mutations in 2 individuals with syndromic intellectual disability and a review of the literature. BMC Med Genet. 2016;17:15. doi:10.1186/s12881-016-0276-4

26. Murray CR, Abel SN, McClure MB, et al. Novel Causative Variants in DYRK1A, KARS, and KAT6A Associated with Intellectual Disability and Additional Phenotypic Features. J Pediatr Genet. 2017;6(2):77-83. doi:10.1055/s-0037-1598639

27. Evers JMG, Laskowski RA, Bertolli M, et al. Structural analysis of pathogenic mutations in the DYRK1A gene in patients with developmental disorders. Hum Mol Genet. 2017;26(3):519-526. doi:10.1093/hmg/ddw409

28. Lee K-S, Choi M, Kwon D-W, et al. A novel de novo heterozygous DYRK1A mutation causes complete loss of DYRK1A function and developmental delay. Sci Rep. 2020;10(1):9849. doi:10.1038/s41598-020-66750-y 
29. Dang T, Duan WY, Yu B, et al. Autism-associated Dyrk1a truncation mutants impair neuronal dendritic and spine growth and interfere with postnatal cortical development. Mol Psychiatry. 2018;23(3):747-758. doi:10.1038/mp.2016.253

30. Qiao F, Shao B, Wang C, et al. A De Novo Mutation in DYRK1A Causes Syndromic Intellectual Disability: A Chinese Case Report. Front Genet. 2019;10:1194. doi:10.3389/fgene.2019.01194

31. Ernst J, Alabek ML, Eldib A, et al. Ocular findings of albinism in DYRK1A-related intellectual disability syndrome. Ophthalmic Genet. Published online August 24, 2020:1-6. doi:10.1080/13816810.2020.1814349

32. Tran KT, Le VS, Bui HTP, et al. Genetic landscape of autism spectrum disorder in Vietnamese children. Sci Rep. 2020;10(1):5034. doi:10.1038/s41598-020-61695-8

33. Møller RS, Kübart S, Hoeltzenbein M, et al. Truncation of the Down syndrome candidate gene DYRK1A in two unrelated patients with microcephaly. Am J Hum Genet. 2008;82(5):1165-1170. doi:10.1016/j.ajhg.2008.03.001

34. Fujita H, Torii C, Kosaki R, et al. Microdeletion of the Down syndrome critical region at 21q22. Am J Med Genet A. 2010;152A(4):950-953. doi:10.1002/ajmg.a.33228

35. Oegema R, de Klein A, Verkerk AJ, et al. Distinctive Phenotypic Abnormalities Associated with Submicroscopic 21q22 Deletion Including DYRK1A. Mol Syndromol. 2010;1(3):113-120. doi:10.1159/000320113

36. Yamamoto T, Shimojima K, Nishizawa T, Matsuo M, Ito M, Imai K. Clinical manifestations of the deletion of Down syndrome critical region including DYRK1A and KCNJ6. Am J Med Genet A. 2011;155A(1):113-119. doi:10.1002/ajmg.a.33735

37. Valetto A, Orsini A, Bertini V, et al. Molecular cytogenetic characterization of an interstitial deletion of chromosome 21 (21q22.13q22.3) in a patient with dysmorphic features, intellectual disability and severe generalized epilepsy. Eur J Med Genet. 2012;55(5):362-366. doi:10.1016/j.ejmg.2012.03.011

38. Kim O-H, Cho H-J, Han E, et al. Zebrafish knockout of Down syndrome gene, DYRK1A, shows social impairments relevant to autism. Mol Autism. 2017;8:50. doi:10.1186/s13229-017-0168-2

39. Meissner LE, Macnamara EF, D'Souza P, et al. DYRK1A pathogenic variants in two patients with syndromic intellectual disability and a review of the literature. Mol Genet Genomic Med. Published online November 7, 2020:e1544. doi:10.1002/mgg3.1544

40. Matsumoto N, Ohashi H, Tsukahara M, Kim KC, Soeda E, Niikawa N. Possible narrowed assignment of the loci of monosomy 21 -associated microcephaly and intrauterine growth retardation to a 1.2-Mb segment at 21q22.2. Am J Hum Genet. 1997;60(4):997-999. 


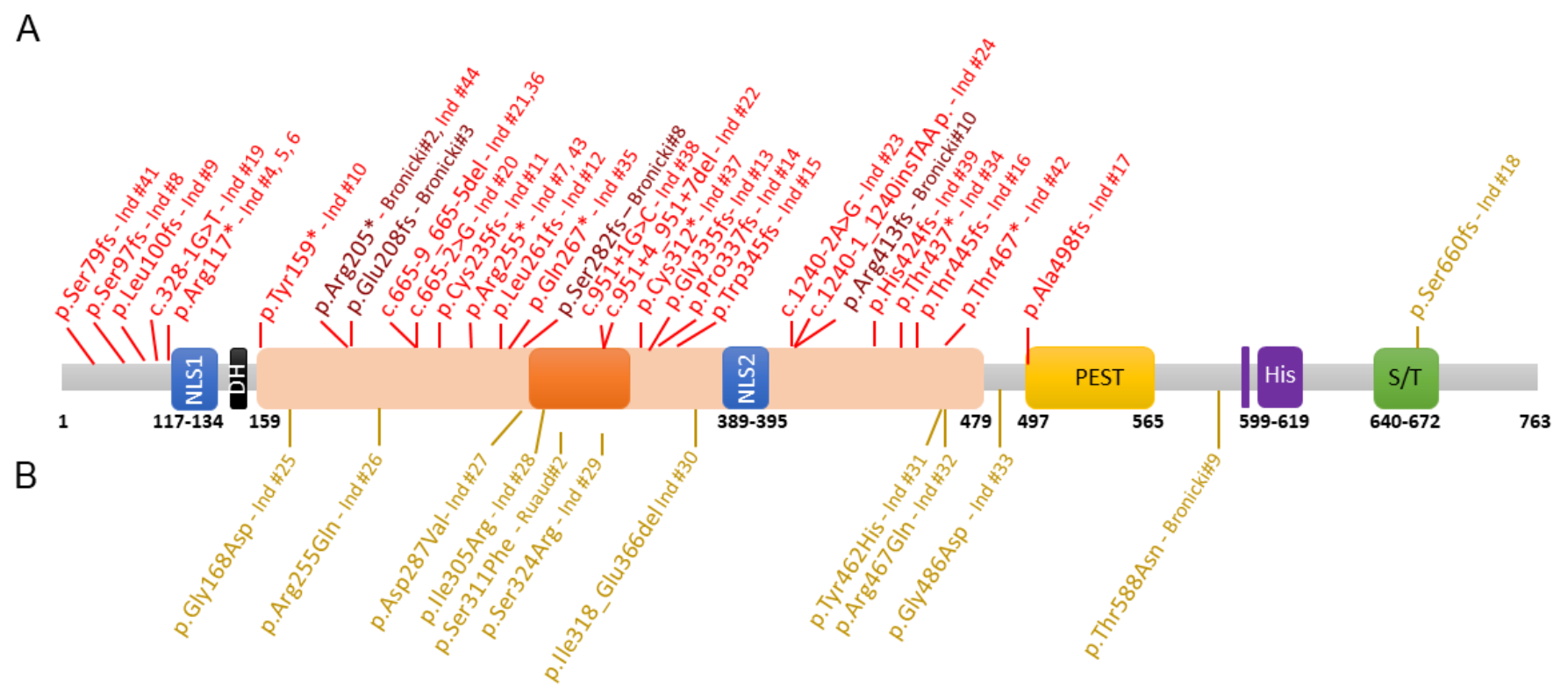

Figure S1 
A $\quad$ c.328-1G>T

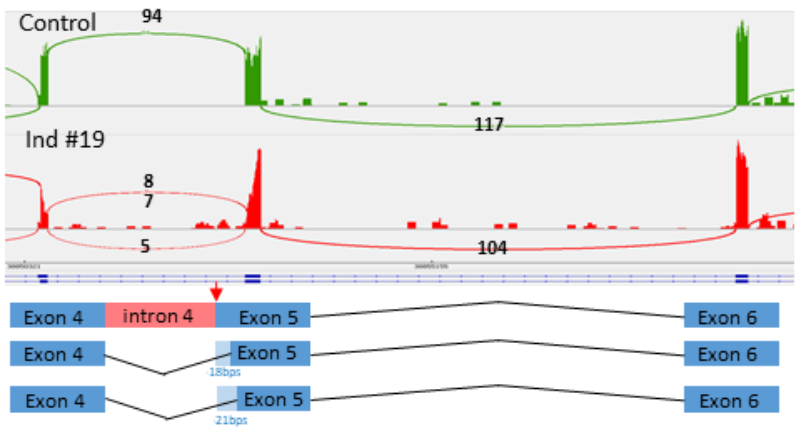

B c.951+4_951+7del

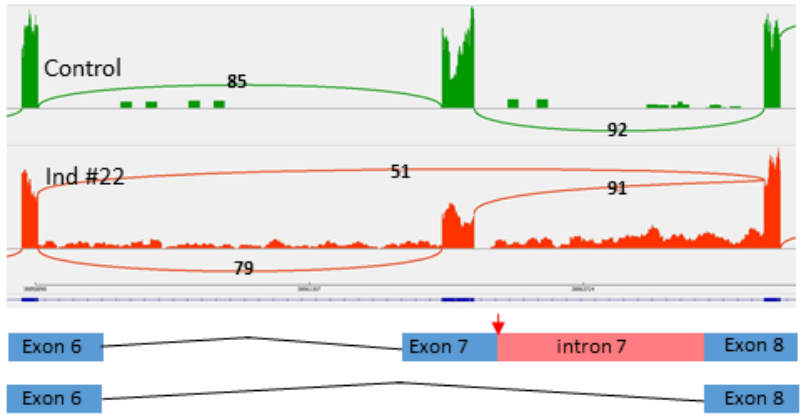

C c.1240-1_1240insTAA

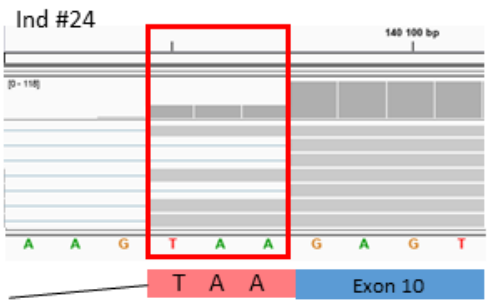

D

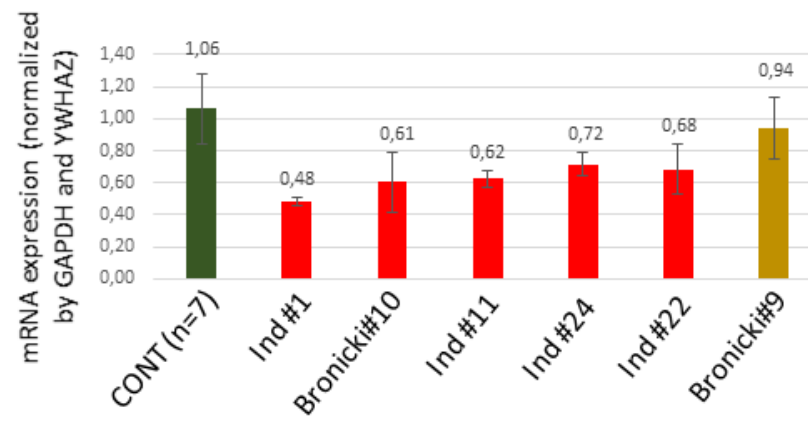

E c.1232dup, p.(Arg413fs)

G AC C A A A G A T G G A A A AC G G G AGT AC A A AC C Bronicki\#10 cDNA (fibroblasts)

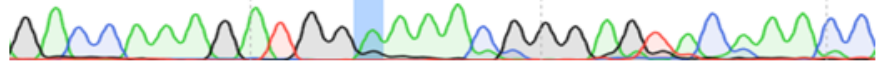

Bronicki\#10 cDNA (fibroblasts treated with emetin)

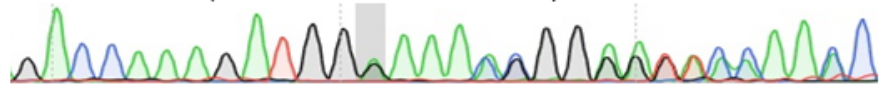

F c.1978del, p.(Ser660fs)

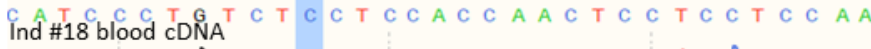

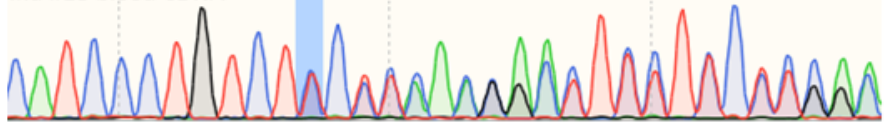

G c.1098G>T

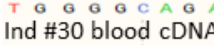

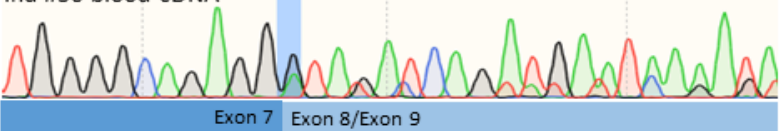

Figure S2 

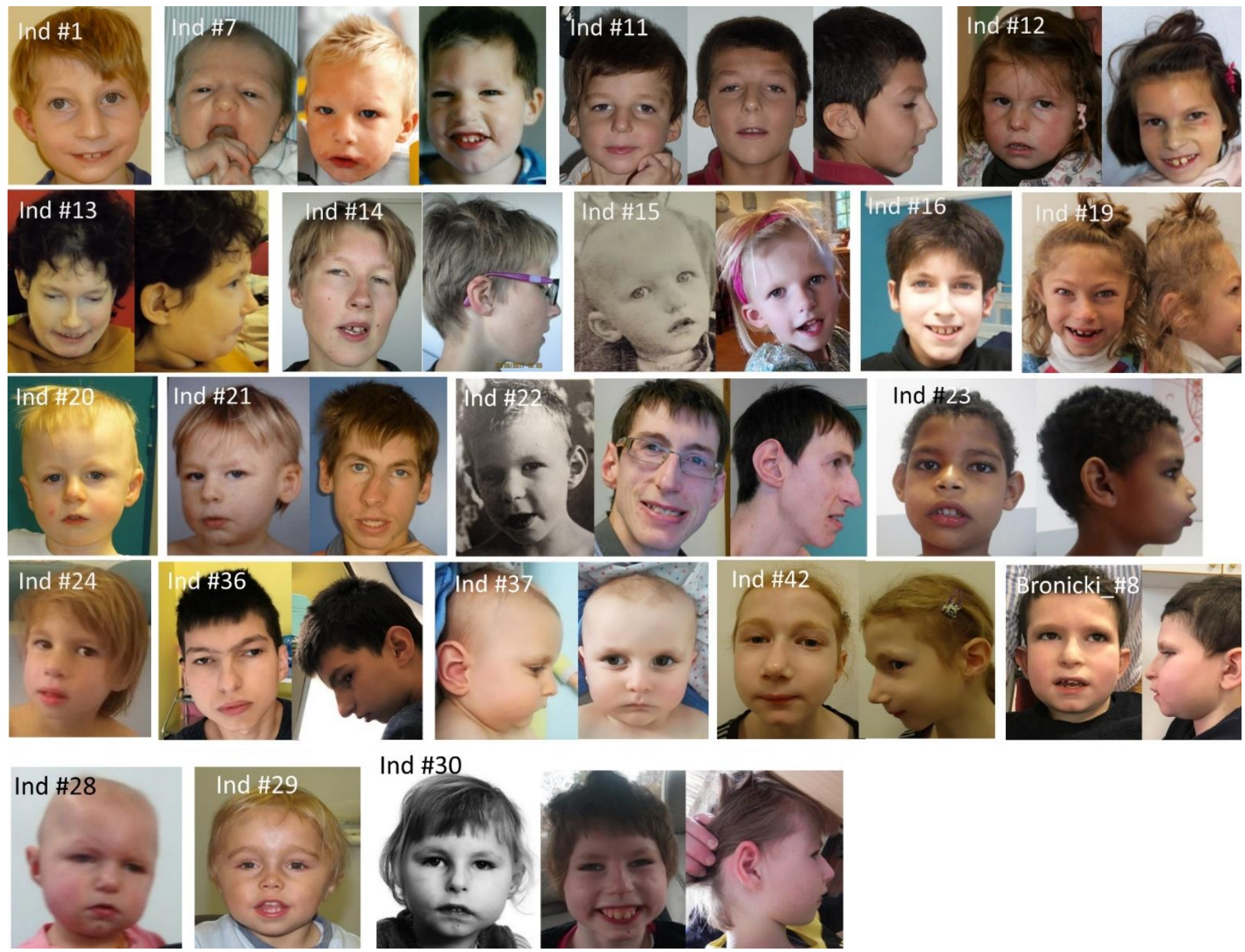

Figure S3 


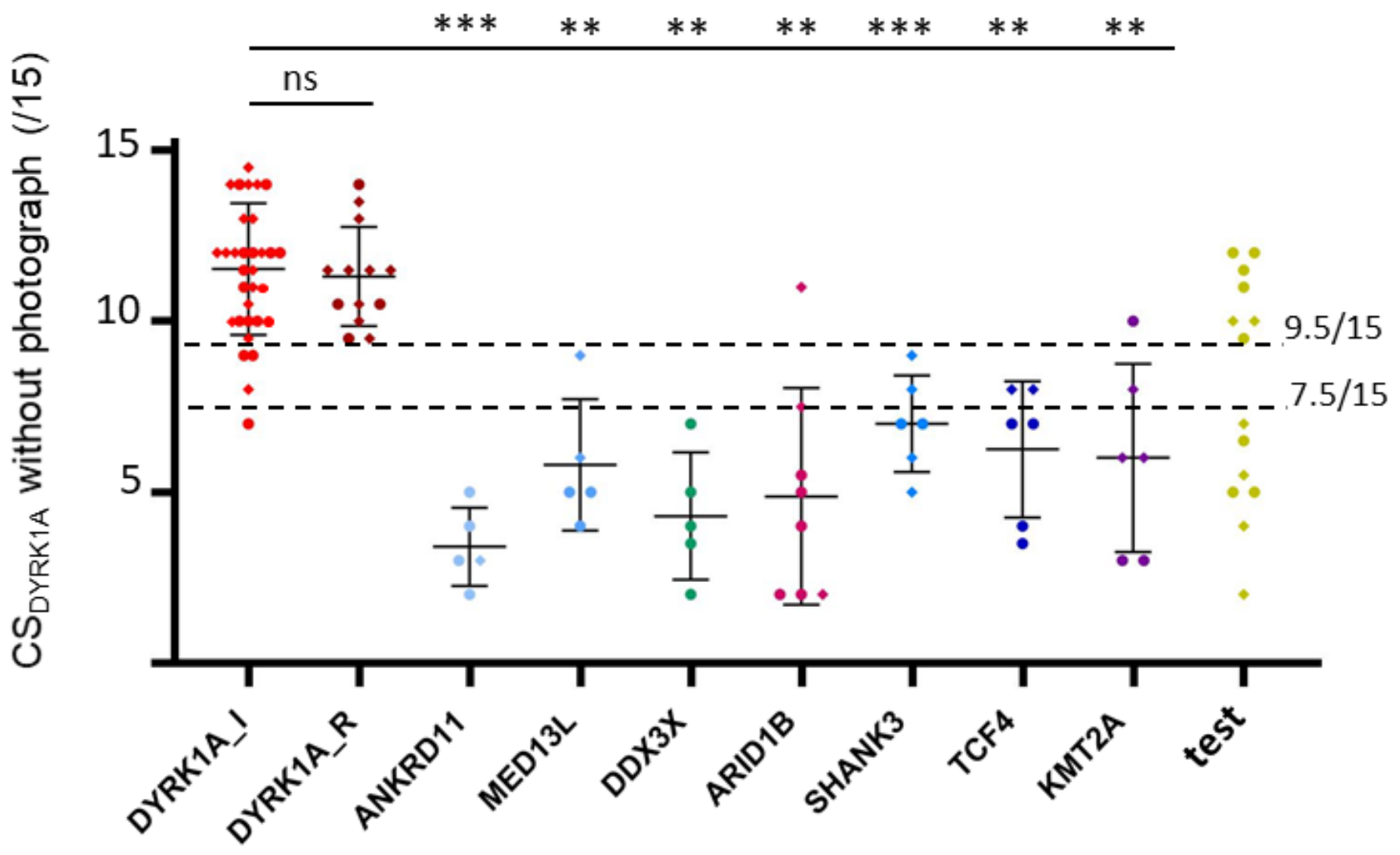

Figure S4

A

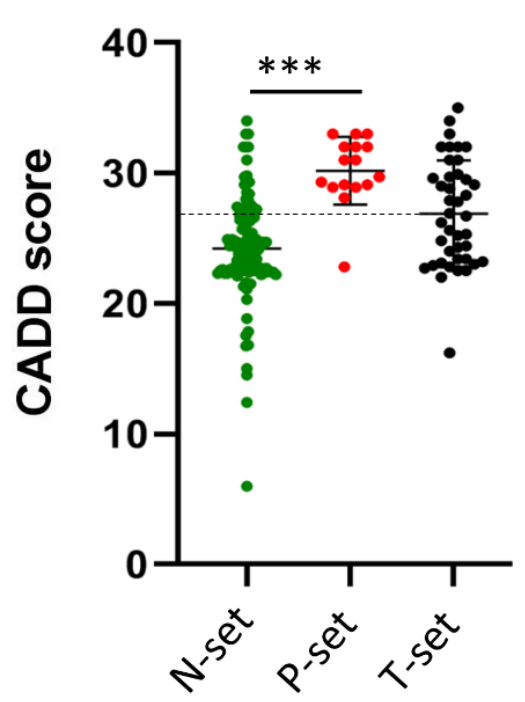

B

\begin{tabular}{|c|c|c|c|c|c|c|}
\hline & \multicolumn{3}{|c|}{ CADD score } & \multicolumn{3}{|c|}{ Conservation } \\
\hline & $>=\mathbf{2 0}$ & $>=25$ & $>=30$ & $V=100 \%$ & $\begin{array}{l}V_{=100 \%} \\
M_{>=90 \%}\end{array}$ & $\begin{array}{c}V=100 \% \\
M_{>}=90 \% \\
O_{>=80 \%}\end{array}$ \\
\hline $\begin{array}{l}\text { N-set } \\
(n=115)\end{array}$ & $\begin{array}{c}92,2 \% \\
(106)\end{array}$ & $\begin{array}{c}32,2 \% \\
(37)\end{array}$ & $\begin{array}{c}7 \% \\
(8)\end{array}$ & $\begin{array}{c}27,8 \% \\
(32)\end{array}$ & $\begin{array}{l}5 \% \\
(6)\end{array}$ & $\begin{array}{c}0,9 \% \\
\left(1^{a}\right)\end{array}$ \\
\hline $\begin{array}{l}\text { P-set } \\
(n=16)\end{array}$ & $\begin{array}{c}100 \% \\
(16)\end{array}$ & $\begin{array}{c}93,75 \% \\
(15) \\
\end{array}$ & $\begin{array}{c}50 \% \\
(8)\end{array}$ & $\begin{array}{c}93,75 \% \\
(15)\end{array}$ & $\begin{array}{c}87,5 \% \\
(14) \\
\end{array}$ & $\begin{array}{c}81,25 \% \\
\left(13^{b}\right)\end{array}$ \\
\hline $\begin{array}{l}\text { T-set } \\
(n=44)\end{array}$ & $\begin{array}{c}97,8 \% \\
(43)\end{array}$ & $\begin{array}{c}61,4 \% \\
\text { (27) }\end{array}$ & $\begin{array}{c}22,7 \% \\
(10)\end{array}$ & $\begin{array}{c}70,5 \% \\
\text { (31) }\end{array}$ & $\begin{array}{c}27,3 \% \\
\text { (12) }\end{array}$ & $\begin{array}{c}22,7 \% \\
\left(10^{c}\right)\end{array}$ \\
\hline
\end{tabular}

Figure S5 


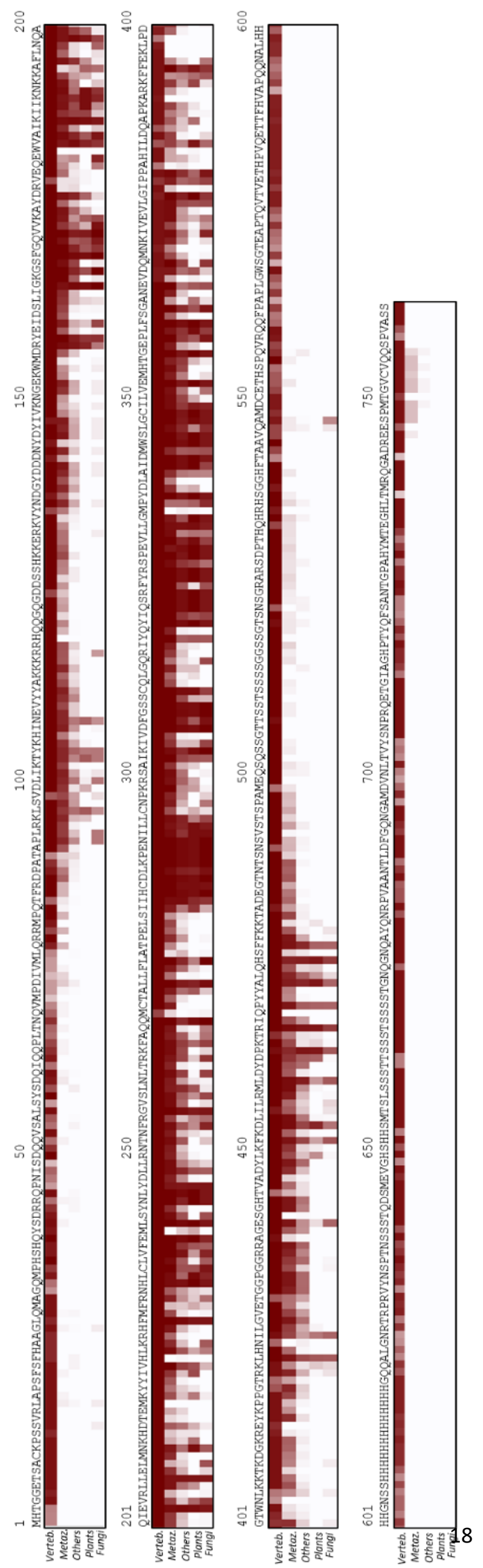

Figure S6 
A

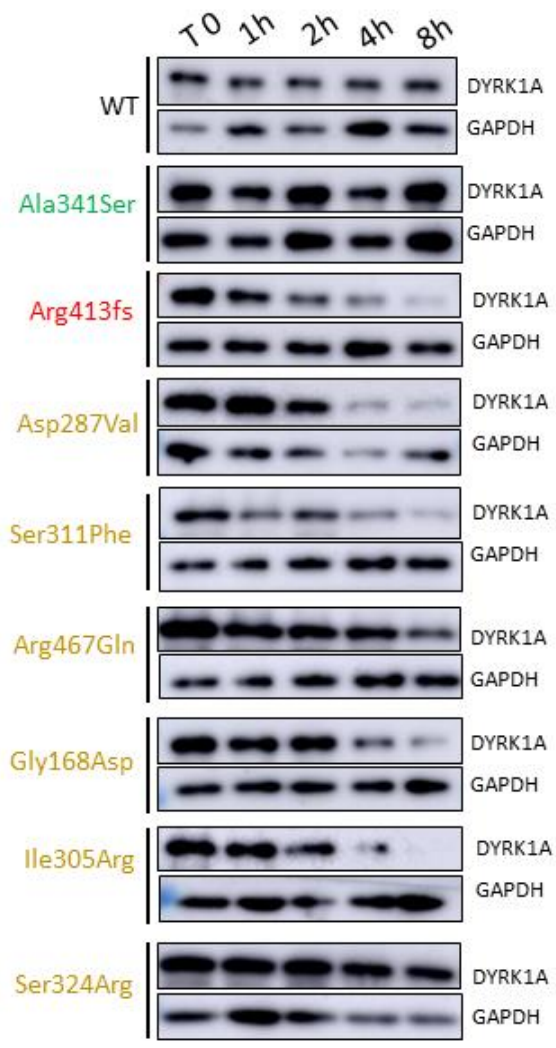

B

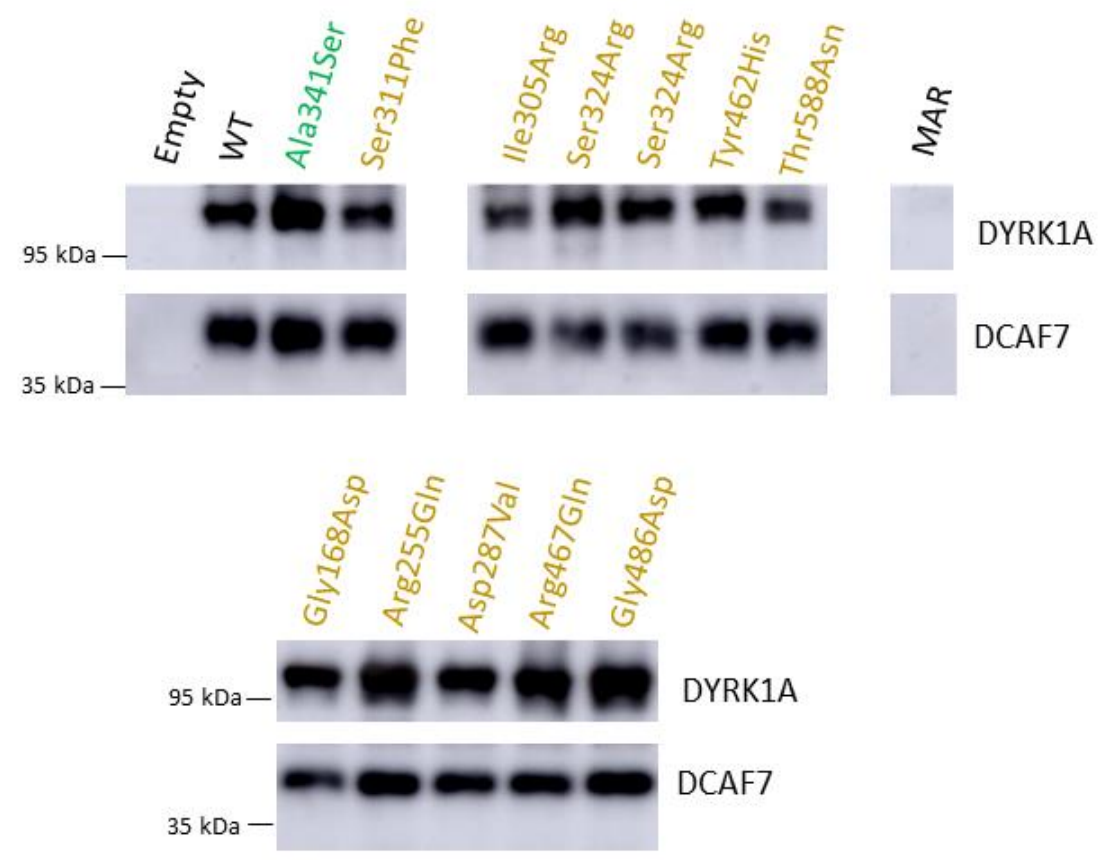

Figure S7 
A

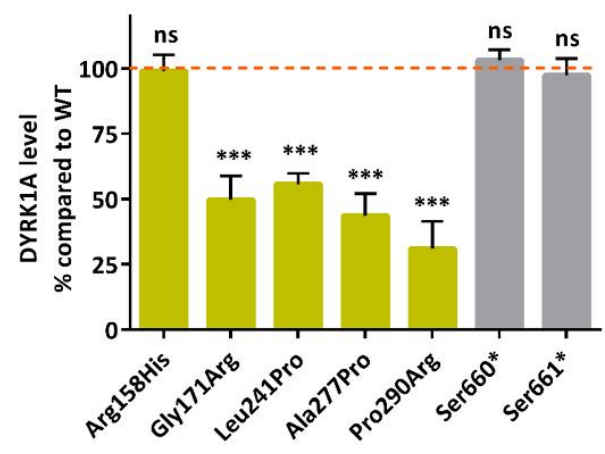

B

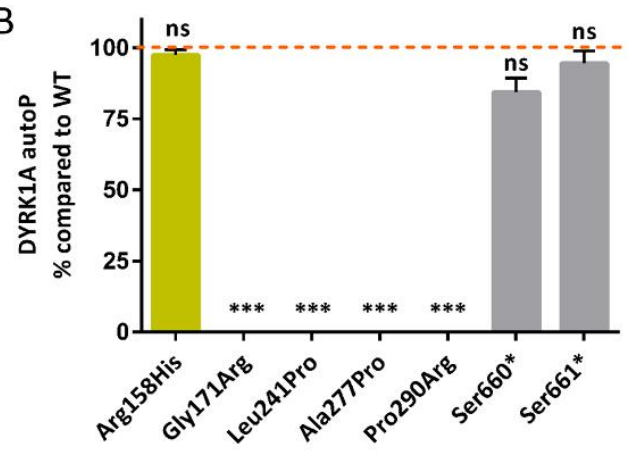

C

$\square$ Mostly cytoplasmic
$\square$ Nuclear and cytoplasmic
$\square$ Mostly nuclear
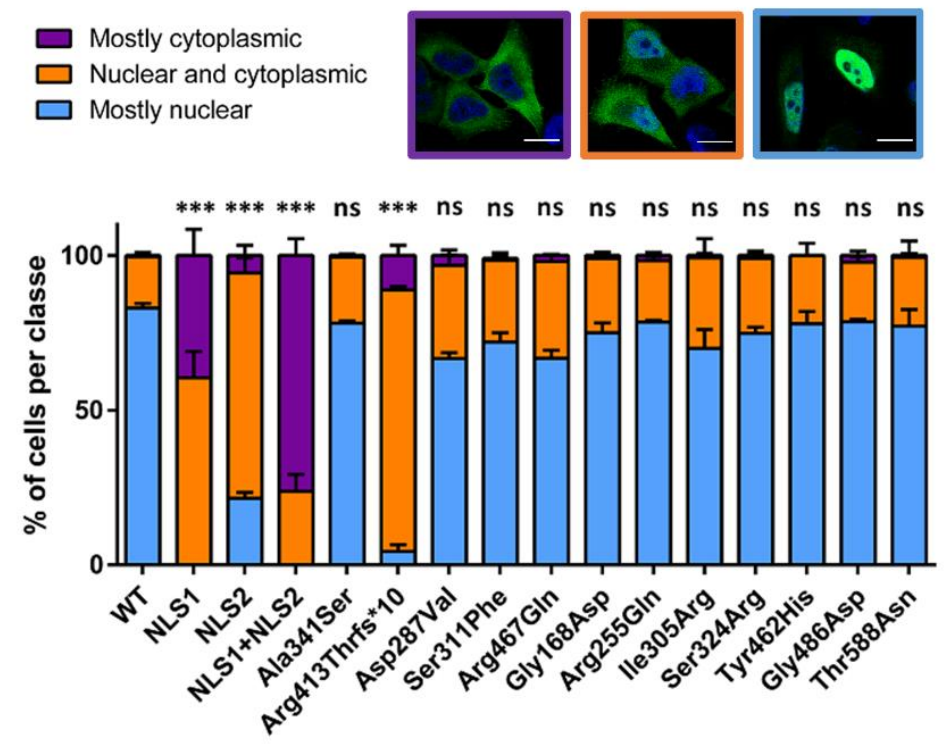

D

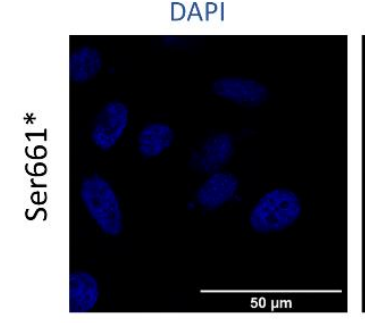

FLAG (DYRK1A)

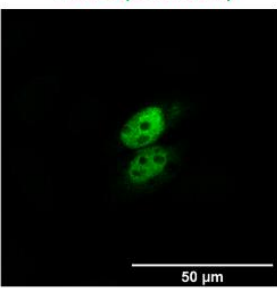

Merge

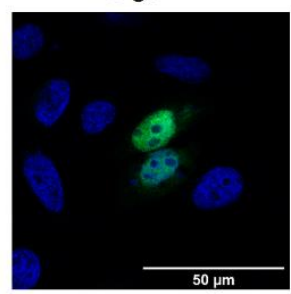

Figure S8 


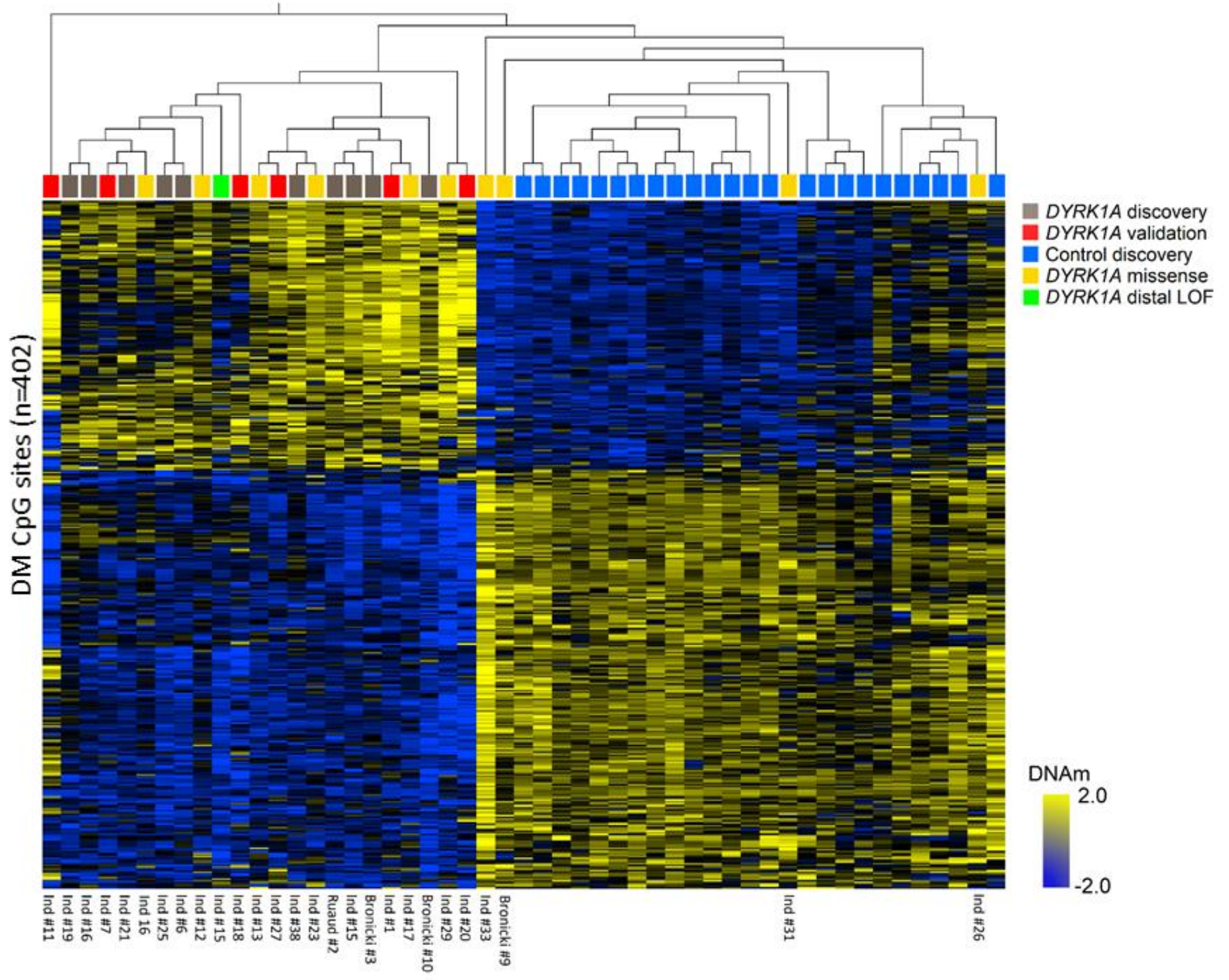

Figure S9 
A

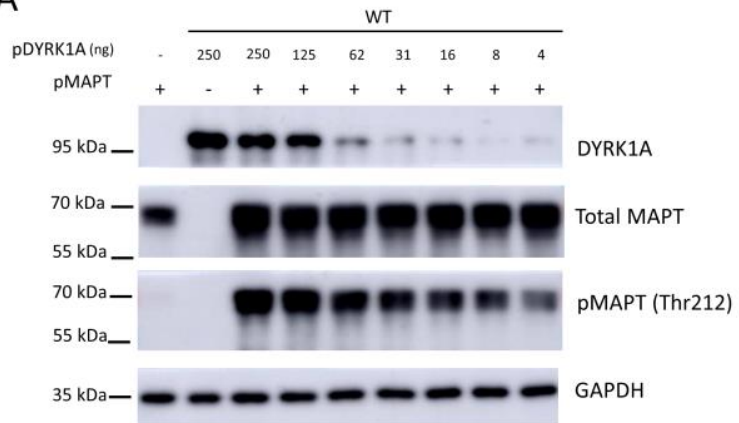

\section{B}

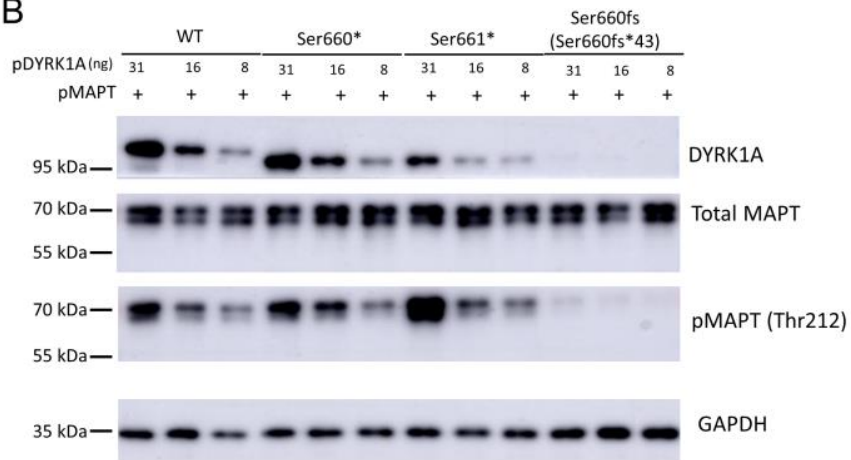

C

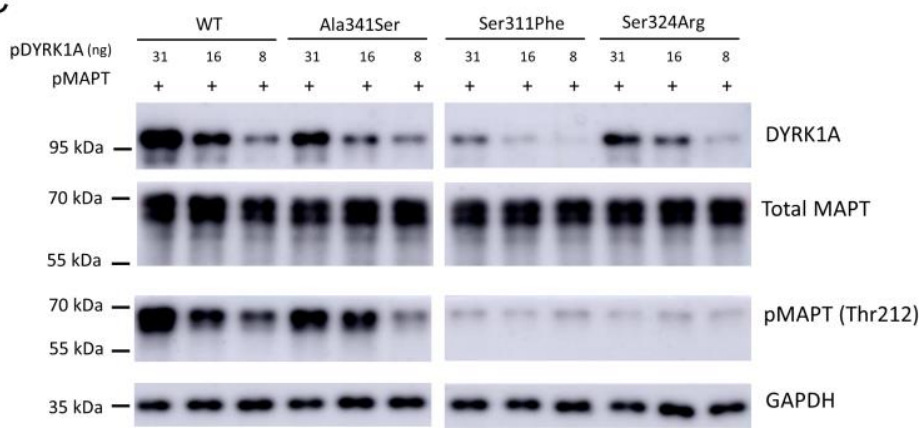

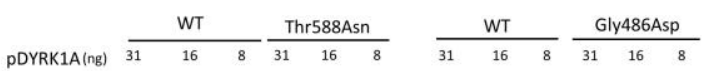

PMAPT +

$95 \mathrm{kDa}-\infty \cdots \cdots \cdots \cdots$

$70 \mathrm{kDa}-\mathrm{kg}$
$55 \mathrm{kDa}$

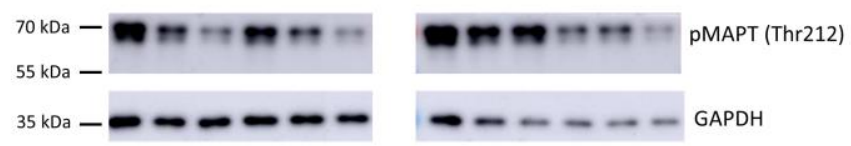

Figure S10 


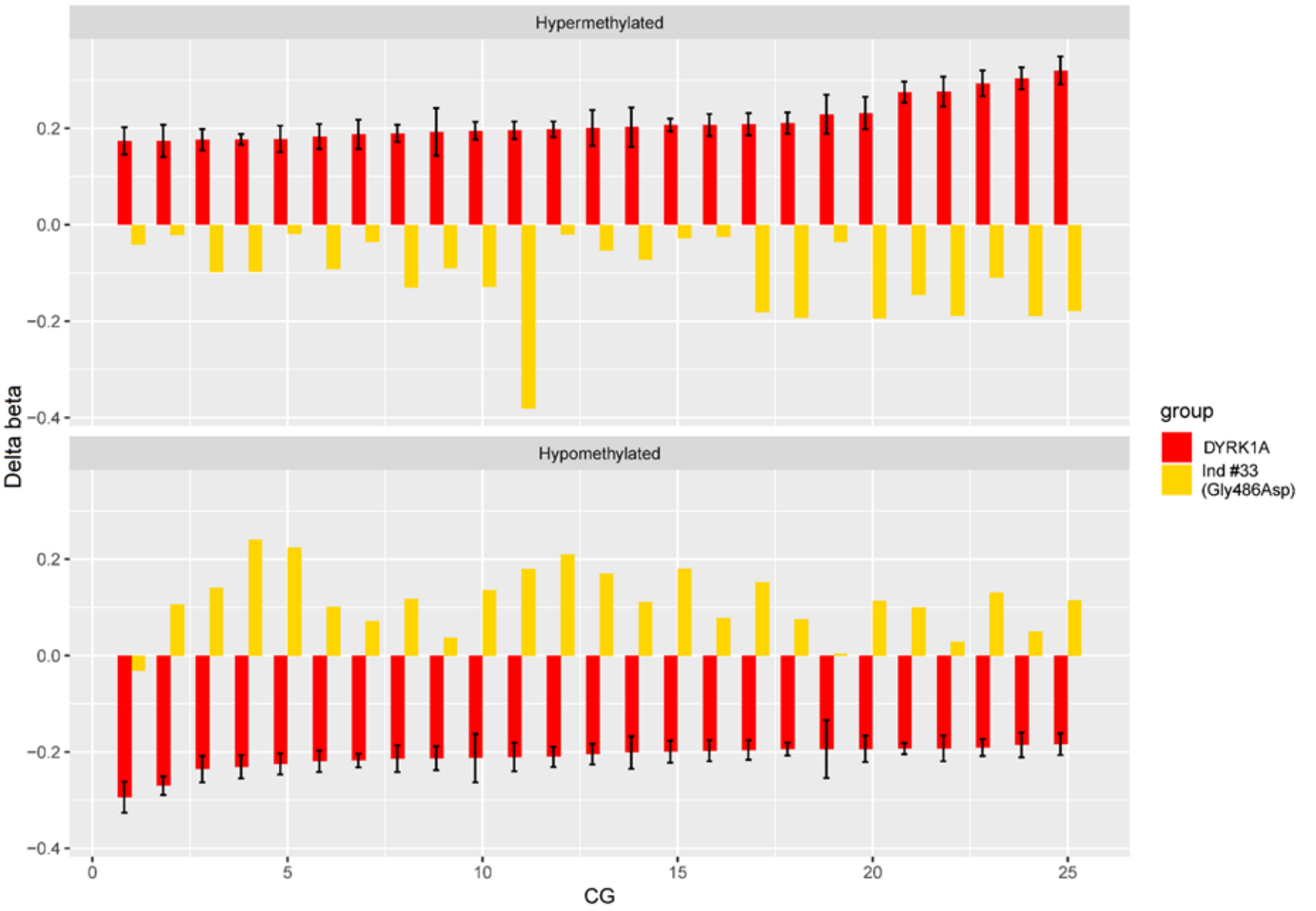

Figure S11 
A

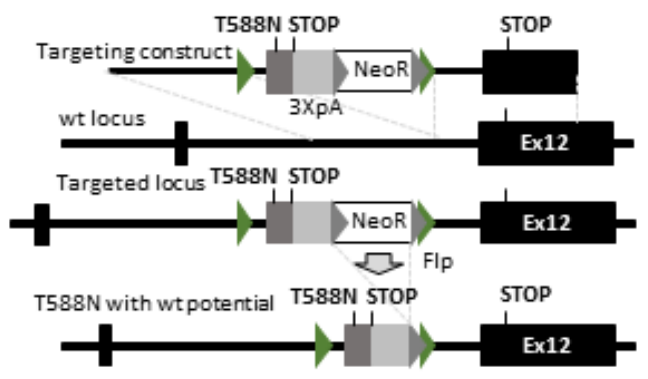

B

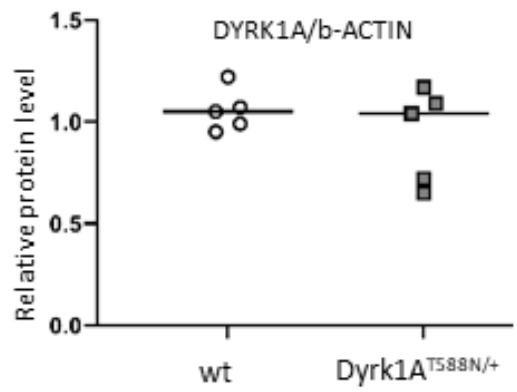

D
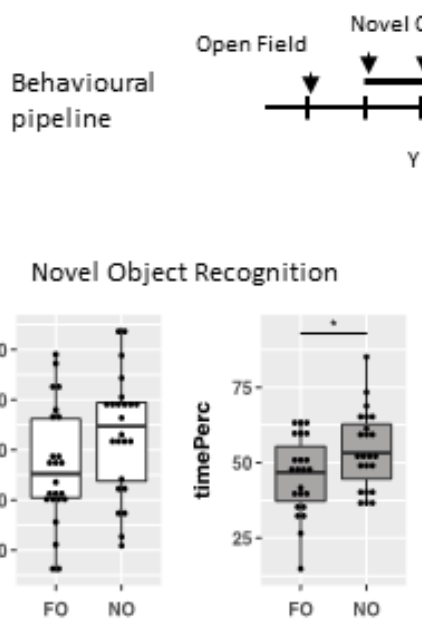

$\mathrm{F}$

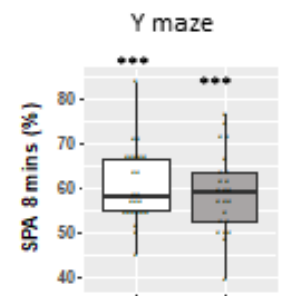

G

Repetitive Behaviour

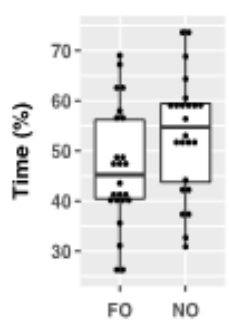

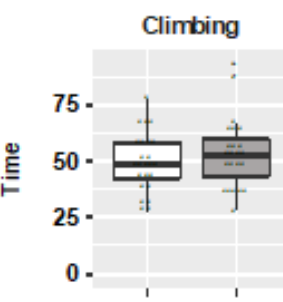

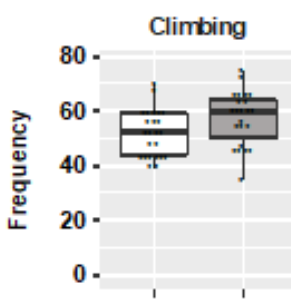

Social 3-chambers test

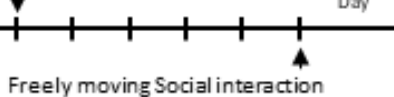

Digging Rearing |
C

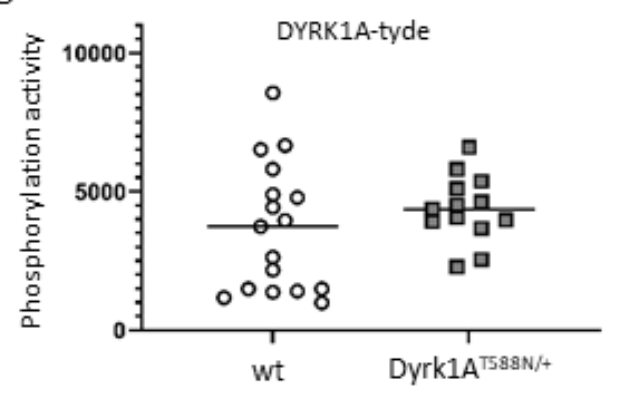

Genotype

官 wt

追 Dyrk1 $1 a^{T S S g N /+}$

$\mathrm{H}$

Social discrimination
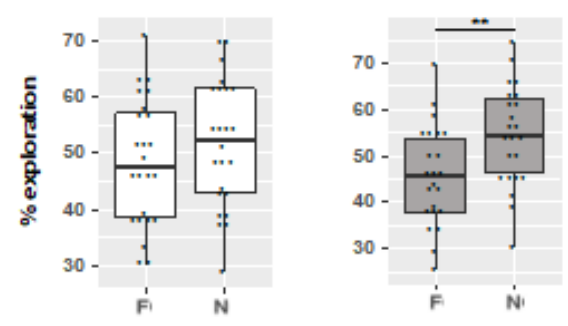

Social interaction

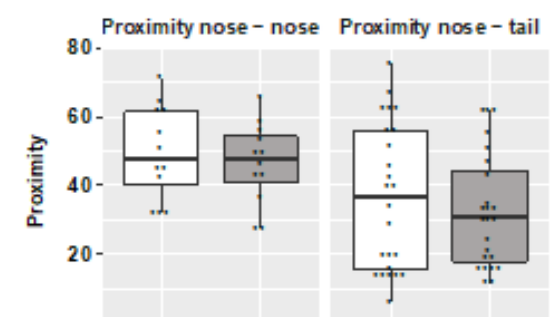

Figure S12 


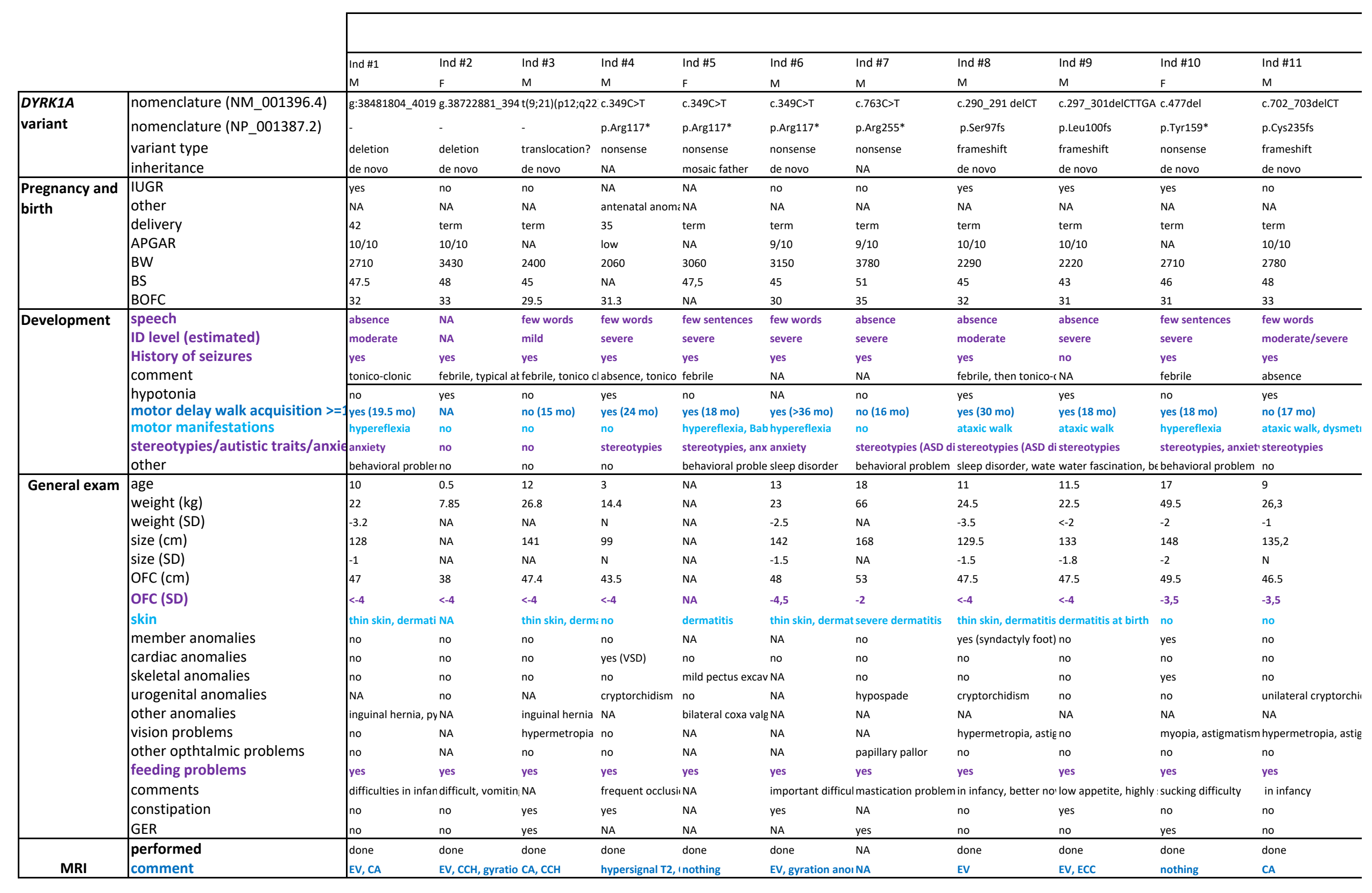

\section{ACCEPTED MANUSCRIPT - CLEAN COPY}




\begin{tabular}{|c|c|c|c|c|c|c|c|c|c|c|c|c|}
\hline \multicolumn{13}{|c|}{ DYRK1A_I } \\
\hline Ind \#12 & Ind \#13 & Ind \#14 & Ind \#15 & Ind \#16 & Ind \#17 & Ind \#19 & Ind \#20 & Ind \#21 & Ind \#22 & Ind \#23 & Ind \#24 & Ind \#34 \\
\hline $\mathrm{F}$ & $\mathrm{F}$ & $\mathrm{F}$ & $\mathrm{F}$ & $\mathrm{F}$ & $\mathrm{F}$ & $\mathrm{F}$ & M & M & M & M & $\mathrm{F}$ & $\mathrm{F}$ \\
\hline c.782del & c.1004del & c.1008dup & c.1033del & c.1333dup & c.1491delC & $c .328-1 \mathrm{G}>\mathrm{T}$ & c.665-2A>G & \multicolumn{3}{|c|}{ c.665-9_665-5delTT(c.951+4_951+7delG7c.1240-2A>G } & \multicolumn{2}{|c|}{ c.1240-1_1240insTA,c.1309C>T } \\
\hline p.Leu261fs & p. Gly335fs & p.Pro337fs & p.Trp345fs & p.Thr445fs & p.Ala498fs & p.? & p.? & p.? & p.? & p.? & p.Glu414* & p.Arg437* \\
\hline frameshift & Frameshift & frameshift & frameshift & frameshift & frameshift & splice & splice & splice & splice & splice & nonsense & nonsense \\
\hline de novo & absent in the mothe & elde novo & de novo & de novo & de novo & de novo & de novo & de novo & de novo & de novo & de novo & NA \\
\hline no & yes & no & yes & yes & yes & no & no & no & yes & yes & yes & yes \\
\hline cerebellar anomaly & NA & NA & CGH-array + CMV + MRI & NA & NA & NA & NA & NA & NA & NA & caesarian & single umbilical arter \\
\hline term & 38 & term & term & 37 & 36 & term & term & 39 & NA & term & term & term \\
\hline $10 / 10$ & NA & NA & NA & $10 / 10$ & 9 & 10 & NA & $10 / 10$ & NA & NA & $10 / 10$ & $10 / 10$ \\
\hline 3030 & 2400 & 3550 & 1865 & 1970 & 1990 & 3450 & 3110 & 3085 & 2690 & \multicolumn{2}{|c|}{3160} & 24102450 \\
\hline 46 & 42 & 52 & 42 & 43,5 & 43 & 49 & 52 & 50 & 47 & \multicolumn{2}{|c|}{48} & 4547 \\
\hline 32 & 30.5 & NA & 29.5 & 30 & 28.5 & 32 & 33 & 34 & NA & \multicolumn{2}{|c|}{33} & 3131 \\
\hline absence & few words & few words & few words/few sentenct & cfew words & few sentences & few words & absence & few sentences & few words & absence & \multirow{2}{*}{$\begin{array}{l}\text { few sentences } \\
\text { moderate }\end{array}$} & few sentences \\
\hline severe & severe & moderate & moderate to severe & severe & severe & severe & moderate & moderate & severe & severe & & mild \\
\hline yes & yes & yes & no & yes & no & yes & yes & yes & yes & yes & \multirow{2}{*}{\multicolumn{2}{|c|}{$\begin{array}{l}\text { yes } \quad \text { yes } \\
\text { febrile seizures + oth febrile }\end{array}$}} \\
\hline NA & atonic & febrile seizures & NA & febrile, then tonico- $\mathrm{c}$ & - - NA & febrile & febrile & tonico-clonic & febrile & febrile & & \\
\hline no & no & no & yes & yes & yes & yes & yes & no & NA & & NA & yes \\
\hline yes (19 mo) & yes $(>36 \mathrm{mo})$ & yes $(>36 \mathrm{mo})$ & yes ( $24 \mathrm{mo})$ & yes $(28 \mathrm{mo})$ & no (16 mo) & yes $(36 \mathrm{mo})$ & yes $(31 \mathrm{mo})$ & no (16.5 mo) & yes $(18 \mathrm{mo})$ & yes $(18 \mathrm{mo})$ & yes $(22 \mathrm{mo})$ & yes $(23 \mathrm{mo})$ \\
\hline & no & ataxic walk & hypereflexia & hypereflexia & NA & \multicolumn{2}{|c|}{ ataxic walk, hyperef NA } & \multicolumn{2}{|c|}{ ataxic walk, hyperef NA } & no & hypereflexia & no \\
\hline stereotypies & stereotypies & stereotypies & stereotypies & stereotypies, anxiet & $t$ ' stereotypies & stereotypies & stereotypies & \multicolumn{4}{|c|}{ stereotypies (ASD di stereotypies, anxiet stereotypies (ASD di stereotypies } & stereotypies, anxiet' \\
\hline sleep disorder, beha & ano & no & sleep disorder & behavioral problem & NA & behavioral problem, & , water fascination & \multicolumn{2}{|c|}{ behavioral problem no } & \multicolumn{3}{|c|}{ sleep disorder, anxie sleep disorder, beha' NA } \\
\hline 7.5 & 27 & 18.5 & 2 & 12.5 & 3.5 & 11 & 1.5 & 20 & NA & 7 & NA & 13 \\
\hline 16.5 & 67 & 47.3 & 7.6 & 30 & 10.9 & 28.1 & 11.6 & 41 & NA & 18.1 & 11 & 27 \\
\hline-2 & NA & -2.7 & NA & -2 & -2.5 & NA & 1 & -4 & NA & -2 & -3 & -2 \\
\hline 118 & 150 & 164 & 76 & 140 & 93.5 & 149 & 82 & 172.5 & NA & 114.5 & NA & 130 \\
\hline-1 & NA & -0.88 & NA & -2 & -2 & NA & 0 & -0.5 & NA & -1.3 & -2 & -2 \\
\hline 46 & 48.5 & 50.5 & 40.5 & 48 & 44.5 & 47.5 & 45 & 51 & NA & 45 & 43 & 47 \\
\hline$<-4$ & $-3,5$ & $<-3$ & -4 & $<-4$ & & $4<-4$ & -3 & -4 & -4 & $<-4$ & -5 & -5 \\
\hline no & NA & thin skin & no & thin skin & thin skin, dermat & is dermatitis at birth & thin skin & \multicolumn{2}{|c|}{ thin skin, dermatitis no } & thin skin & thin skin & thin skin, dermatitis \\
\hline no & no & short 5 th metatars, & Eves (adductus thumb) & no & NA & no & no & \multicolumn{2}{|c|}{ yes. Bilateral postaxi no } & no & NA & no \\
\hline no & NA & no & no & yes (AtSD) & NA & no & NA & no & no & no & NA & no \\
\hline no & scoliosis,eqinus feet & $t$ abnormalities of th & & no & NA & no & no & no & no & no & NA & no \\
\hline NA & anal malposition & no & no & no & NA & NA & NA & no & cryptorchidism & no & NA & grade II vesicoureter \\
\hline inguinal hernia, vas & o hiatal hernia & Acrocyanosis - han & NA & NA & NA & NA & NA & NA & inguinal hernia & NA & NA & velopalatine insuffici \\
\hline astigmatism & NA & myopia & NA & myopia & hypermetropia & no & NA & \multicolumn{2}{|c|}{ myopia, astigmatism myopia } & NA & NA & NA \\
\hline no & NA & no & no & no & NA & papillary pallor & papillary pallor & \multicolumn{2}{|c|}{ hypolasia optic nervino } & no & no & hypolasia optic nervt \\
\hline yes & yes & yes & yes & yes & yes & yes & yes & no & yes & yes & yes & yes \\
\hline difficult in infancy & gastrostomy & breast, additional & NA & important feeding di & ii very difficult beth & first months & low appetite & NA & highly selective & very selective & NA & very selective \\
\hline no & no & no & yes & yes & yes & yes & no & no & yes & no & yes & yes \\
\hline no & yes & NA & $\mathrm{NA}$ & no & NA & yes & NA & no & NA & no & NA & yes \\
\hline done & done & done & done & done & done & done & done & done & not done & done & done & done \\
\hline $\mathrm{CCH}, \mathrm{CeA}$ (pre and $\mathrm{p}$ & $p$ EV & nothing & NA & CCH, atrophy CSE & delayed myelinat & $\mathrm{OCCCH}, \mathrm{CA}, \mathrm{EV}$ & $\mathrm{EV}, \mathrm{CA}$ & EV & NA & $\mathrm{CA}, \mathrm{CeA}$ & nothing & enlarged pericerebri \\
\hline
\end{tabular}




\begin{tabular}{|c|c|c|c|c|c|c|c|c|c|c|c|c|c|}
\hline \multirow[b]{2}{*}{ Ind\#35 } & \multirow[b]{2}{*}{ Ind\#36 } & \multirow[b]{2}{*}{ Ind \#37 } & \multirow[b]{2}{*}{ Ind \#38 } & \multirow[b]{2}{*}{ Ind\#39 } & \multirow[b]{2}{*}{ Ind \#40 } & \multirow[b]{2}{*}{ Ind \#41 } & \multirow[b]{2}{*}{ Ind \#42 } & \multirow[b]{2}{*}{ Ind \#43 } & \multirow[b]{2}{*}{ Ind \#44 } & \multicolumn{2}{|c|}{ Total $(n=34)$} & \multicolumn{2}{|c|}{$\begin{array}{c}\text { Previously } \\
\text { reported }^{\mathrm{a}}(\mathrm{n}=80)\end{array}$} \\
\hline & & & & & & & & & & Total & $\%$ & Total & $\%$ \\
\hline M & M & $\mathrm{F}$ & $\mathrm{M}$ & $\mathrm{F}$ & $\mathrm{M}$ & $\mathrm{F}$ & $\mathrm{F}$ & $\mathrm{F}$ & $\mathrm{M}$ & $17 \mathrm{~F} / 17 \mathrm{M}$ & & & \\
\hline c.799C>T & \multicolumn{2}{|c|}{ c.665-9_665-5delTT(c.936T>A } & $951+1 G>C$ & c.1270del & \multicolumn{2}{|c|}{ g.38302140_400414 c.235dupA } & $c .1399 C>T$ & c.763C>T & c.613C>T & & & & \\
\hline p.Gln267* & p.? & p.Cys312* & p.? & p.His424fs & - & p.Arg79fs & p.Arg467* & p.Arg255* & p.Arg205* & & & & \\
\hline nonsense & splice & nonsense & splice & frameshift & deletion & frameshift & nonsense & nonsense & nonsense & & & & \\
\hline de novo & de novo & de novo & de novo & de novo & de novo & de novo & de novo & NA & NA & & & & \\
\hline no & yes & yes & no & yes & yes & yes & yes & yes & yes & $20 / 32$ & $63 \%$ & $22 / 32$ & $69 \%$ \\
\hline NA & NA & NA & NA & NA & NA & NA & NA & NA & kidney asymetry & & & & \\
\hline term & term & term & term & term & term & term & term & 35 & term & & & & \\
\hline \multirow[t]{4}{*}{ NA } & $10 / 10$ & NA & $10 / 10$ & $9 / 10$ & $10 / 10$ & $10 / 10$ & 9/10 & NA & NA & & & & \\
\hline & 2580 & 2175 & 2810 & 2700 & 3395 & 2160 & 2530 & 1695 & 2590 & & & & \\
\hline & 44 & 43 & 49 & 47 & 46 & 43.6 & 43 & 43.5 & 44 & & & & \\
\hline & 33 & 30,2 & 33 & 31.5 & NA & 30 & 31.5 & 28.5 & 32 & & & & \\
\hline few sentences & absence & few words & $\mathrm{NA}$ & few sentences & absence & $\mathrm{NA}$ & few words & few words & absence & $30 / 30$ (A/FW: 22/31) & $100 \%(71 \%)$ & 74/74 & $100 \%$ \\
\hline moderate & severe & NA & NA & moderate/severe & severe & NA & severe & severe & severe & $32 / 32$ (M/s:28/30) & $100 \%(93 \%)$ & 73/73 & $100 \%$ \\
\hline yes & yes & yes & NA & yes & yes & yes & yes & yes & no & 29/33 (febrile: 18) & $88 \%$ & \multicolumn{2}{|c|}{ 56/76 (febri $74 \%$} \\
\hline febrile & \multicolumn{2}{|c|}{ febrile + tonicoclonic febrile } & NA & \multicolumn{3}{|c|}{ febrile, absence, my febrile, tonico-clonic NA } & \multicolumn{2}{|c|}{ absence, tonico-clon NA } & NA & & & & \\
\hline yes & yes & yes & yes & no & NA & yes & no & no & NA & $16 / 29$ & $55 \%$ & & \\
\hline yes $(19 \mathrm{mo})$ & yes $(21 \mathrm{mo})$ & yes $(25 \mathrm{mo})$ & yes $(>26 \mathrm{mo})$ & yes $(24 \mathrm{mo})$ & yes $(28 \mathrm{mo})$ & yes $(21 \mathrm{mo})$ & no (17 mo) & yes $(20 \mathrm{mo})$ & yes $(>36 \mathrm{mo})$ & $27 / 33$ & $82 \%$ & $59 / 67$ & $88 \%$ \\
\hline no & NA & digitigrade walk & hypereflexia & no & pyramidal signs & pyramidal signs, hy & hypertonia & NA & NA & $18 / 28$ & $64 \%$ & $32 / 44$ & $73 \%$ \\
\hline stereotypies & \multicolumn{2}{|c|}{ stereotypies, anxiet' stereotypies } & NA & anxiety & stereotypies, anxi & tiNA & anxiety & no & no & $28 / 32$ & $88 \%$ & $40 / 45$ & $89 \%$ \\
\hline sleep disorder & behavioral probler & water fascination, & $\in \mathrm{NA}$ & NA & sleep disorder, wa & sleep disorders & sleep disorders, $v$ & tabsence of fear & no & & & & \\
\hline 23 & 18 & 3 & NA & 13 & 8.5 & 5 & 15 & 6 & 4 & & & & \\
\hline 63 & 40,5 & 12 & NA & 31.6 & 19 & 16.7 & 36.6 & 15.8 & 13.1 & & & & \\
\hline $\mathrm{N}$ & NA & -1 & NA & -1.3 & -2 & NA & -2.5 & -1.4 & -1.7 & & & & \\
\hline 173 & 159,5 & 87 & NA & 139 & 120 & 102 & 150.5 & 113 & 95 & & & & \\
\hline $\mathrm{N}$ & NA & -2 & NA & -1.7 & -1.5 & NA & -2 & $\mathrm{~N}$ & -2 & & & & \\
\hline 52.5 & 52 & 44 & NA & 50 & 45.5 & 45 & 49.3 & 44 & 44.5 & & & & \\
\hline-3 & -3 & $-3,5$ & NA & -2 & -6 & $<-3$ & $<-3$ & $<-4$ & $<-4$ & $32 / 32$ & $100 \%$ & 72/77 & $94 \%$ \\
\hline no & no & thin skin & NA & NA & thin skin & NA & thin skin & no & NA & $19 / 28$ & $68 \%$ & 10/? & \\
\hline no & no & yes & NA & long fingers, small t & hno & NA & no & no & no & 7 & & & \\
\hline no & no & NA & NA & pulmonary valve $d y$ & & NA & no & no & no & 3 & & & \\
\hline cyphosis & scoliosis & pectus & NA & no & no & NA & no & no & no & 7 & & & \\
\hline testicular ectopia & no & ovarian hernia & NA & no & NA & NA & no & no & micropenis & $10 / 23$ & $43 \%$ & 13/? & \\
\hline NA & NA & NA & NA & no & nail dystrophy & NA & no & no & no & & & & \\
\hline myopia & hypermetropia & no & NA & hypermetropia & NA & NA & hypermetropia & no & hypermetropia, astig & $15 / 21$ & $71 \%$ & & \\
\hline no & no & no & strabism & nystagmus & hypolasia optic ne & «NA & no & strabism & no & & & & \\
\hline no & yes & yes & yes & yes & yes & NA & yes & yes & yes & $31 / 33$ & $94 \%$ & $50 / 57$ & $88 \%$ \\
\hline NA & smashed food & NA & NA & first months & poor eating & NA & NA & very selective & NA & & & & \\
\hline yes & no & no & NA & NA & no & NA & yes & no & yes & & & 12/? & \\
\hline no & no & yes & yes & yes & no & NA & yes & no & NA & & & $11 / ?$ & \\
\hline not done & done & done & NA & NA & done & done & done & done & done & & & & \\
\hline NA & periventricular wh & tinA & NA & NA & $\mathrm{CCH}$, enlarged cist & $r$ enlarged pericerebr & nothing & $\mathrm{CA}$ & $\mathrm{CCH}$ & $22 / 27$ & $81 \%$ & $39 / 52$ & $75 \%$ \\
\hline
\end{tabular}

\section{ACCEPTED MANUSCRIPT - CLEAN COPY}




\begin{tabular}{|c|c|c|c|c|c|c|c|c|c|c|c|c|c|c|c|}
\hline \multicolumn{4}{|c|}{ DYRK1A_R (Bronicki et al., 2014) } & \multicolumn{12}{|c|}{ Individuals with variants to test } \\
\hline Bronicki_\#2 & Bronicki_\#3 & Bronicki_\#8 & Bronicki_\#10 & Ind \#18 & Ind \#25 & Ind \#26 & Ruaud \#2 & Ind \#29 & Ind \#31 & Ind \#30 & Ind \#28 & Ind \#32 & Ind \#27 & Ind \#33 & Bronicki_\#9 \\
\hline M & M & M & $\mathrm{F}$ & $\mathrm{F}$ & $\mathrm{F}$ & $\mathrm{F}$ & M & M & M & $\mathrm{F}$ & $\mathrm{F}$ & $\mathrm{F}$ & M & M & $\mathrm{F}$ \\
\hline$c .613 C>T$ & \multicolumn{2}{|c|}{ c.621_624 delin: c.844dupA } & c.1232dup & c.1978del & $c .503 \mathrm{G}>\mathrm{A}$ & c.764G>A & $c .932 \mathrm{C}>\mathrm{T}$ & c.972T>A & c.1384T>C & $\begin{array}{l}c .1098 G>T \\
\end{array}$ & $c .914 \mathrm{~T}>\mathrm{G}$ & c. $1400 \mathrm{G}>\mathrm{A}$ & $c .860 \mathrm{~A}>\mathrm{T}$ & c.1457G>A & c.1763C>A \\
\hline p.Arg205* & p.Glu208fs & \multicolumn{2}{|c|}{ p.(Ser282Lysfs* ${ }^{*}$ p.Arg413fs } & p.Ser660fs & p.Gly168Asp & p.Arg255Gln & p.Ser311Phe & p.Ser324Arg & p.Tyr462His & \multicolumn{2}{|c|}{ p.lle318_Glu36€ p.lle305Arg } & p.Arg 467Gln & p.Asp287Val & p.Gly486Asp & p.Thr588Asn \\
\hline nonsense & frameshift & frameshift & frameshift & frameshift & missense & missense & missense & missense & missense & inframe del & missense & missense & missense & missense & missense \\
\hline de novo & de novo & de novo & de novo & de novo & de novo & NA & de novo & de novo & de novo & de novo & de novo & de novo & de novo & de novo & de novo \\
\hline $\mathrm{NA}$ & NA & no & yes & yes & NA & no & yes & yes & NA & yes & yes & NA & yes & NA & no \\
\hline NA & NA & NA & NA & \multicolumn{2}{|c|}{ heart ventricula NA } & NA & artery & NA & NA & NA & NA & NA & NA & NA & NA \\
\hline term & term & term & term & term & NA & term & term & term & term & term & term & NA & prematurity & NA & term \\
\hline $10 / 10$ & $10 / 10$ & $8 / 10 / 10$ & $10 / 10$ & $10 / 10$ & NA & $10 / 10$ & NA & NA & $7 / 10$ & $9 / 10$ & NA & NA & NA & NA & $8 / 3$ \\
\hline 2720 & 3280 & 3290 & 2215 & 2660 & NA & 3490 & 3170 & 2575 & 3440 & 2570 & 2550 & NA & 2200 & NA & 3000 \\
\hline 47 & 49 & 47 & 46 & 46 & NA & 51,5 & 49 & 46 & 50,5 & 48 & 47 & NA & NA & NA & 48 \\
\hline 32 & 33 & 34 & 32 & 31 & NA & 36 & 33.5 & 33 & 37 & 31 & 31.5 & NA & NA & NA & 32 \\
\hline few words & few words & absence & few words & \multicolumn{2}{|c|}{ few sentences/INA } & few words & few words & few sentences & normal & absence & few words & NA & few words & normal & absence \\
\hline moderate & moderate & \multicolumn{2}{|c|}{ moderate/seveı moderate/seve } & moderate & NA & NA & moderate & mild & mild & severe & severe & NA & severe & noID & severe \\
\hline yes & absence? & yes & yes & no & NA & no & yes & yes & yes & yes & yes & no & yes & no & yes \\
\hline NA & NA & \multicolumn{2}{|c|}{ febrile, tonico-cl febrile, myoclon } & NA & NA & NA & febrile & atonic & febrile & \multicolumn{2}{|c|}{ febrile, tonic-clo febrile } & NA & febrile & NA & absence, tonic \\
\hline NA & NA & yes & NA & no & NA & no & NA & yes & no & yes & NA & NA & no & NA & \\
\hline yes & yes $(23 \mathrm{mo})$ & no $(16 \mathrm{mo})$ & yes $(20 \mathrm{mo})$ & yes $(18 \mathrm{mo})$ & NA & yes $(18 \mathrm{mo})$ & yes & yes $(22 \mathrm{mo})$ & no $(13 \mathrm{mo})$ & yes $(36 \mathrm{mo})$ & yes & NA & yes $(20 \mathrm{mo})$ & no & yes $(>36 \mathrm{mo})$ \\
\hline NA & hypertonia & hypereflexia & NA & no & NA & no & NA & no & normal & no & \multicolumn{2}{|c|}{ ataxic walk, hyp NA } & NA & no & ataxia \\
\hline anxiety & stereotypies & ASD diagnosis & flapping & anxiety & NA & stereotypies & stereotypies & ASD, anxiety & anxiety & sterotypies & sterotypies & sterotypies & stereotypies & ASD & stereotypies \\
\hline NA & sleep disorders & \multicolumn{2}{|c|}{ water fascinatio NA } & NA & NA & \multicolumn{2}{|c|}{ behavioral problNA } & \multicolumn{2}{|c|}{ sleep disorder, b aggresivity } & \multicolumn{4}{|c|}{ sleep disorder, water fascination, behavioral prob NA } & NA & sleep disorder, $\mathrm{v}$ \\
\hline 15 & 3 & NA & NA & 12 & NA & 3 & NA & 5 & 22,5 & 16 & NA & $\mathrm{NA}$ & 6 & 10 & 38 \\
\hline 43.8 & 13.5 & NA & NA & 39 & NA & 15,7 & NA & 11,5 & 89 & 44 & NA & NA & 21 & 39.4 & 75 \\
\hline NA & -1 & NA & -2 & $\mathrm{~N}$ & NA & $\mathrm{N}$ & NA & +1.2 & NA & -2.3 & -1.5 & NA & NA & NA & +3.5 \\
\hline 167 & 94 & NA & NA & 154 & NA & NA & NA & 101,5 & 181 & 162 & NA & NA & 117 & 156 & 170 \\
\hline NA & -1 & NA & $-0,5$ & +1.7 & NA & NA & NA & $-1,5$ & NA & -1 & NA & NA & NA & NA & 1 \\
\hline 43,8 & 46 & NA & NA & 50 & NA & NA & NA & 52 & 60,5 & 47 & NA & NA & 46.5 & 58.5 & NA \\
\hline-3 & -4 & $<-3$ & -3.5 & -2.5 & NA & 2 & $<-4$ & +1 & NA & $<-3$ & -4 & NA & $<-3$ & NA & -3 \\
\hline thin skin & NA & no & thin skin & no & NA & no & NA & no & no & no & NA & dermatitis & NA & +2 & thin skin \\
\hline no & NA & no & NA & no & NA & no & no & \multicolumn{2}{|c|}{ clinodactyly 5th no } & \multicolumn{3}{|c|}{ long fingers, can NA } & flat feet & NA & no \\
\hline no & NA & VSD & NA & no & NA & no & no & no & no & NA & NA & NA & NA & NA & NA \\
\hline pectus excavatu & & scoliosis & NA & NA & NA & no & no & no & scoliosis & NA & NA & NA & NA & NA & NA \\
\hline no & NA & NA & no & no & NA & no & no & no & no & NA & NA & NA & micropenis & NA & no \\
\hline no & NA & NA & NA & NA & NA & no & no & no & no & NA & NA & NA & NA & NA & NA \\
\hline hypermetropia & hypermetropia, & hypermyopia & NA & hypermetropia & NA & no & NA & NA & hypermyopia & NA & NA & NA & NA & NA & NA \\
\hline NA & & NA & NA & no & NA & NA & no & NA & no & NA & NA & NA & NA & NA & NA \\
\hline yes & yes & yes & yes & no & NA & no & no & yes & no & yes & NA & yes & NA & NA & yes \\
\hline NA & NA & gastrostomy & NA & NA & NA & NA & no & NA & NA & \multicolumn{2}{|c|}{ difficulty during no } & NA & NA & NA & mild \\
\hline NA & yes & yes & NA & no & NA & no & NA & yes & no & yes & NA & NA & yes & NA & yes \\
\hline NA & $\mathrm{NA}$ & yes & NA & no & NA & no & $\mathrm{NA}$ & yes & no & no & NA & NA & NA & NA & no \\
\hline done & done & done & done & done & NA & done & done & done & done & done & done & done & NA & NA & not done \\
\hline EV, plagiocepha & aEV & EV & $\mathrm{EV}, \mathrm{CCA}$ & thin optic nerve & eNA & nothing & EV & nothing & EV & $\mathrm{CA}, \mathrm{EV}$ & $\mathrm{CCH}$ & normal & NA & NA & NA \\
\hline
\end{tabular}

\section{ACCEPTED MANUSCRIPT - CLEAN COPY}




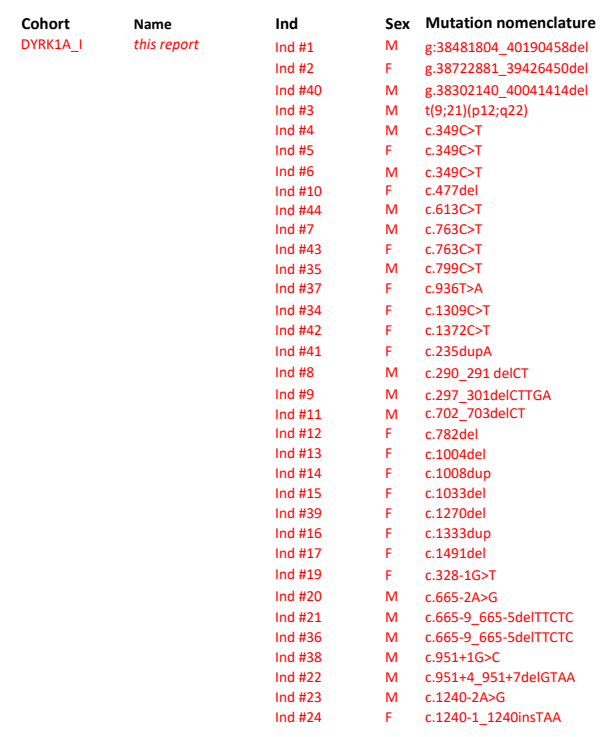

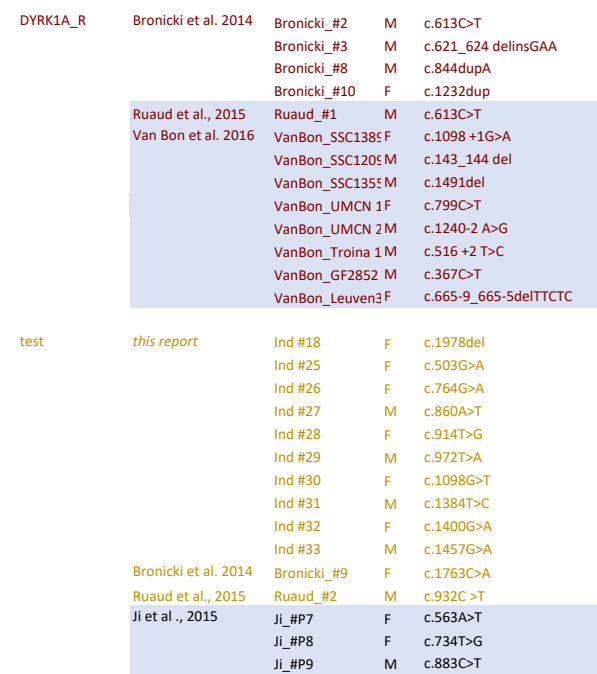

\begin{tabular}{|c|c|c|c|c|}
\hline \multirow{5}{*}{\multicolumn{2}{|c|}{$\begin{array}{l}\text { other monogenic ANKRD11 } \\
\text { forms }\end{array}$}} & ANKRD11 1 & $\mathrm{~F}$ & pathogenic variant \\
\hline & & ANKRD11_2 & $\mathrm{F}$ & pathogenic variant \\
\hline & & ANKRD11_3 & M & pathogenic variant \\
\hline & & ANKRD11_4 & $\mathrm{F}$ & pathogenic variant \\
\hline & & ANKRD11_5 & F & pathogenic variant \\
\hline \multirow{4}{*}{\multicolumn{2}{|c|}{ MED13L }} & MED13L_1 & $M$ & pathogenic variant \\
\hline & & $\begin{array}{l}\text { MEE13L_2 } \\
\text { MED1LL 3 }\end{array}$ & & $\begin{array}{l}\text { pathogenenic varlant } \\
\text { pathogenic variant }\end{array}$ \\
\hline & & MED13L 4 & & pathogenic variant \\
\hline & & MED13L_5 & $\mathrm{F}$ & pathogenic variant \\
\hline \multirow{5}{*}{\multicolumn{2}{|c|}{ DDX3X }} & DDX3X_1 & $\mathrm{F}$ & pathogenic variant \\
\hline & & DDX3X_2 & $\mathrm{F}$ & pathogenic variant \\
\hline & & DDX3X_3 & $\mathrm{F}$ & pathogenic variant \\
\hline & & DDX3X 4 & $F$ & pathogenic variant \\
\hline & & DDX3X_5 & $\mathrm{F}$ & pathogenic variant \\
\hline \multirow{8}{*}{\multicolumn{2}{|c|}{ ARID1B }} & ARID1B_1 & $\mathrm{F}$ & pathogenic variant \\
\hline & & ARID1B_2 & M & pathogenic variant \\
\hline & & ARID1B_3 & $\mathrm{F}$ & pathogenic variant \\
\hline & & ARID1B 4 & $\mathrm{~F}$ & pathogenic variant \\
\hline & & ARID1B 5 & $\mathrm{~F}$ & pathogenic variant \\
\hline & & ARID1B_6 & M & pathogenic variant \\
\hline & & ARID1B_7 & M & pathogenic variant \\
\hline & & ARID1B_8 & $\mathrm{F}$ & pathogenic variant \\
\hline \multirow{6}{*}{\multicolumn{2}{|c|}{ SHANK3 }} & SHANK3 1 & $\mathrm{~F}$ & pathogenic variant \\
\hline & & SHANK3_2 & M & pathogenic variant \\
\hline & & SHANK3_3 & M & pathogenic variant \\
\hline & & SHANK3_4 & M & pathogenic variant \\
\hline & & SHANK3_5 & $\mathrm{F}$ & pathogenic variant \\
\hline & & SHANK3 6 & M & pathogenic variant \\
\hline \multirow{6}{*}{\multicolumn{2}{|c|}{ TCF4 }} & TCF4_1 & M & pathogenic variant \\
\hline & & TCF4_2 & M & pathogenic variant \\
\hline & & TCF4_3 & $\mathrm{F}$ & pathogenic variant \\
\hline & & TCF4_4 & $\mathrm{F}$ & pathogenic variant \\
\hline & & TCF4_5 & $\mathrm{F}$ & pathogenic variant \\
\hline & & TCF4_6 & $\mathrm{F}$ & pathogenic variant \\
\hline \multirow{6}{*}{\multicolumn{2}{|c|}{ KMT2A }} & KMT2A 1 & M & pathogenic variant \\
\hline & & KMT2A_2 & $F$ & pathogenic variant \\
\hline & & KMT2A_3 & M & pathogenic variant \\
\hline & & KMT2A_4 & $\mathrm{F}$ & pathogenic variant \\
\hline & & KMT2A_5 & M & pathogenic variant \\
\hline & & KMT2A_6 6 & $F$ & pathogenic variant \\
\hline
\end{tabular}

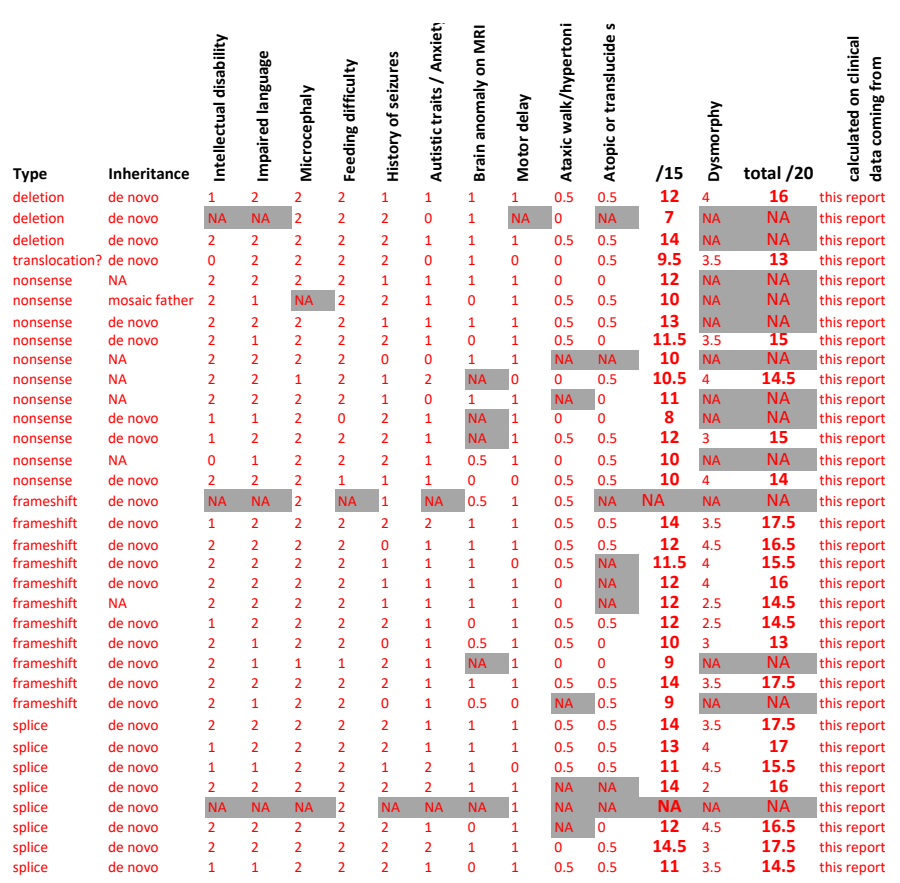

$\begin{array}{llllllllllllllll}\text { p.Arg205* nonsense de novo } & 1 & 2 & 2 & 2 & 0 & 1 & 0 & 1 & \text { NA } & 0.5 & 9.5 & 5 & \mathbf{1 4 . 5} & \text { this report }\end{array}$ $\begin{array}{lllllllllllllll}\text { p.Glu208fs frameshift de novo } & 1 & 2 & 2 & 2 & 1 & 1 & 1 & 1 & 0.5 & \text { NA } & \mathbf{1 1 . 5} & 3.5 & \mathbf{1 5} & \text { this report }\end{array}$ $\begin{array}{llllllllllllllll}\text { p.Ser282fs frameshift de novo } & 2 & 2 & 2 & 2 & 2 & 2 & 1 & 0 & 0.5 & 0 & \mathbf{1 3 . 5} & \mathbf{3} & \mathbf{1 6 . 5} & \text { this report }\end{array}$

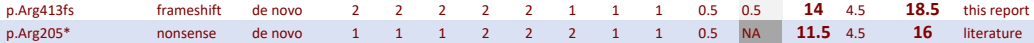
$\begin{array}{llllllllllllllllll} & \text { nonsense } & \text { de novo } & 1 & 1 & 1 & 2 & 2 & 2 & 1 & 1 & 0.5 & \text { NA } & \mathbf{1 1 . 5} & 4.5 & \mathbf{1 6} & \text { ilterature } \\ \text { splice } & \text { de novo } & 2 & 2 & 1 & 2 & 0 & 2 & 0 & \text { NA } & 0.5 & \text { NA } & \mathbf{9 . 5} & 4.5 & \mathbf{1 4} & \text { literature } \\ & & & & \end{array}$

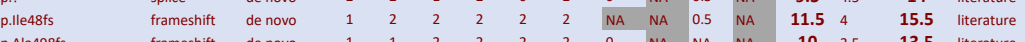

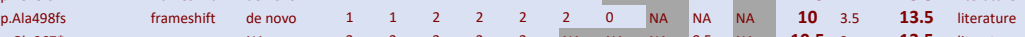

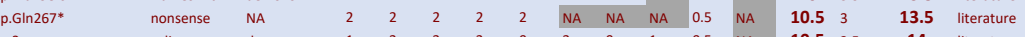
$\begin{array}{lllllllllllllllll}\text { p.? } & \text { splice } & \text { de novo } & 1 & 2 & 2 & 2 & 0 & 2 & 0 & 1 & 0.5 & \text { NA } & \mathbf{1 0 . 5} & 3.5 & \mathbf{1 4} & \text { literature } \\ \text { splice } & \text { de novo } & 2 & 2 & 2 & 2 & 2 & 1 & 1 & \text { NA } & 0.5 & 0.5 & \mathbf{1 3} & 5 & \mathbf{1 8} & \text { literature }\end{array}$ $\begin{array}{lllllllllllllllll}\text { p.? } & \text { splice } & \text { de novo } & 2 & 2 & 2 & 2 & 2 & 1 & 1 & \text { NA } & 0.5 & 0.5 & \mathbf{1 3} & 5 & \mathbf{1 8} & \text { literature } \\ \text { nonsense } & \text { de novo } & 1 & 2 & 2 & 2 & 2 & 2 & 0 & 0 & 0.5 & \text { NA } & \mathbf{1 1 . 5} & 4.5 & \mathbf{1 6} & \text { literature }\end{array}$

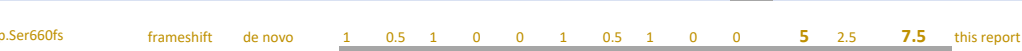

$\begin{array}{lll}\text { frameshift } & \text { de novo } \\ \text { p.Gly168Asp } & \text { missense } & \text { de novo }\end{array}$ missense NA $\begin{array}{lll}\text { m.Asp287Val } & \text { missense } & \text { de novo } \\ \text { p.lle305Arg } & \text { missense de novo }\end{array}$ $\begin{array}{lll}\text { missense } & \text { de novo } \\ \text { p.lle318 Glu366del } & \text { inframe } & \text { de novo }\end{array}$ Tyr462His missense de novo missense de novo $\begin{array}{lll}\text { missense de novo } & \\ \text { missense } & \text { de novo }\end{array}$ p.Leu245Arg

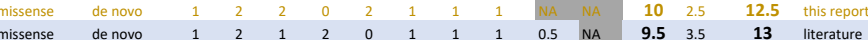
$\begin{array}{llllllllllllllll}\text { missense de novo } & 1 & 2 & 1 & 2 & 0 & 1 & 1 & 1 & 0.5 & \mathrm{NA} & \mathbf{9 . 5} & 3.5 & \mathbf{1 3} & \text { literature }\end{array}$ $\begin{array}{lllllllllllllll}\text { missense de novo } & 2 & 2 & 2 & 2 & 1 & 1 & 0.5 & 1 & 0.5 & \text { NA } & \mathbf{1 2} & \text { NA } & \text { NA literature } \\ \text { missense de novo } & 0 & 2 & 2 & 0 & 0 & 1 & \text { NA } & \text { NA } & 0.5 & \text { NA } & \mathbf{5 . 5} & \text { NA } & \text { NA } & \end{array}$
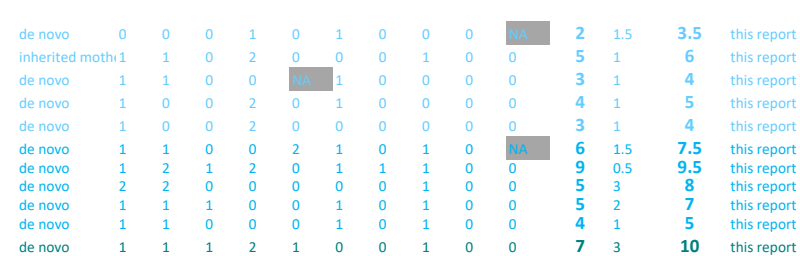

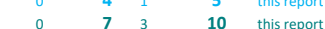

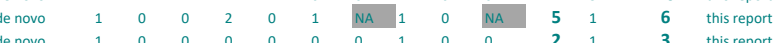

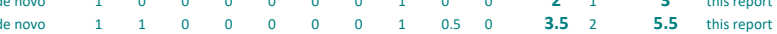

$\begin{array}{lllllllllllllll}\text { de novo } & 1 & 1 & 0 & 0 & 0 & 0 & 1 & 1 & 0 & 0 & 4 & 0.5 & 4.5 & \text { this report }\end{array}$

$\begin{array}{llllllllllllllll}\text { de novo } & 0 & 1 & 0 & 0 & 1 & 2 & \text { NA } & 0 & 0 & 0 & \text { NA } & \mathbf{4} & 1.5 & \mathbf{5 . 5} & \text { this report }\end{array}$

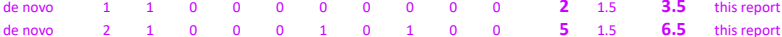

$\begin{array}{lllllllllllllll}\text { de novo } & 1 & 1 & 0 & 0 & 0 & 0 & 0 & 0 & 0 & 0 & \mathbf{2} & 2.5 & \mathbf{4 . 5} & \text { this report } \\ \text { denort }\end{array}$

$\begin{array}{lllllllllllllll}\text { de novo } & 1 & 1 & 0 & 0 & 0 & 0 & 0 & 0 & 0 & 0 & \mathbf{2} & \mathbf{1} & \mathbf{3} & \text { this report } \\ \text { de novo } & 2 & 2 & 0 & 2 & 2 & 1 & 0 & 1 & 0.5 & 0.5 & \mathbf{1 1} & \mathbf{1 . 5} & \mathbf{1 2 . 5} & \text { this report }\end{array}$

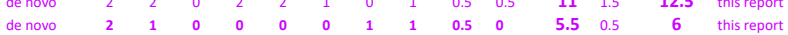

$\begin{array}{llllllllllllllll}\text { de novo } & 2 & 1 & 0 & 2 & 0 & 1 & 0 & 1 & 0 & 0.5 & 7.5 & 1.5 & 9 & \text { this report } \\ \text { this report }\end{array}$

$\begin{array}{lllllllllllllll}\text { de novo } & 2 & 2 & 0 & 0 & 1 & 2 & \text { NA } & 0 & 0 & \text { NA } & \mathbf{7} & 1 & \mathbf{8} & \text { this report } \\ \text { de novo } & 2 & 2 & 1 & 0 & 2 & 0 & 0.5 & 1 & 0.5 & 0 & \mathbf{9} & 1.5 & \mathbf{1 0 . 5} & \text { this report }\end{array}$

$\begin{array}{lllllllllllllll}\text { de novo } & 2 & 2 & 1 & 0 & 2 & 0 & 0.5 & 1 & 0.5 & 0 & \mathbf{9} & 1.5 & \mathbf{1 0 . 5} & \text { this report } \\ \text { de novo } & 1 & 2 & 0 & 2 & 0 & 1 & 0 & 0 & 0 & 0 & \mathbf{6} & 1 & \mathbf{7} & \text { this report }\end{array}$

$\begin{array}{lllllllllllllll}\text { de novo } & 2 & 1 & 0 & 0 & 1 & 1 & 0 & 0 & 0 & 0 & 5 & 3 & 8 & \text { this report } \\ \text { de novo } & 2 & 2 & 0 & 0 & 1 & 1 & 0 & 1 & 0 & 0 & 7 & 1.5 & 8.5 & \\ \text { this report }\end{array}$

$\begin{array}{llllllllllllllll}\text { de novo } & \\ \text { de novo } & 2 & 2 & 0 & 0 & 1 & 2 & \text { NA } & 1 & 0 & 0 & 8 & 1 & 9 & \text { this report } \\ \text { this report }\end{array}$

$\begin{array}{lllllllllllllll}\text { de novo } & 2 & 2 & 0 & 0 & 1 & 1 & 0.5 & 1 & 0.5 & 0 & \mathbf{8} & \mathbf{3} & \mathbf{1 1} & \text { this report } \\ \text { dis report } & \end{array}$

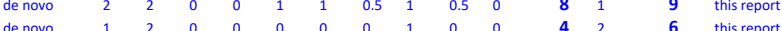

$\begin{array}{lllllllllllllll}\text { de novo } & 2 & 2 & 0 & 0 & 0 & 1 & 0.5 & 1 & 0.5 & 0 & \mathbf{7} & 1.5 & \mathbf{8 . 5} & \text { this report }\end{array}$

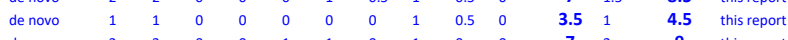

$\begin{array}{llllllllllllllll}\text { de novo } & 2 & 2 & 0 & 0 & 1 & 1 & 0 & 1 & 0 & 0 & \mathbf{7} & 2 & \mathbf{9} & \text { this report }\end{array}$

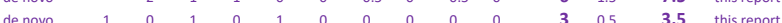

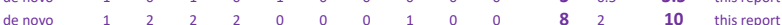

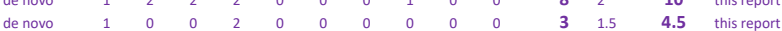

$\begin{array}{lllllllllllllrl}\text { de novo } & 1 & 0 & 0 & 2 & 0 & 0 & 0 & 0 & 0 & 0 & \mathbf{3} & 1.5 & \mathbf{4 . 5} & \text { this report } \\ \text { de novo } & 1 & 1 & 0 & 2 & 0 & 1 & 0 & 1 & 0 & 0 & \mathbf{6} & \mathbf{2} & \mathbf{8} & \text { this report }\end{array}$ 


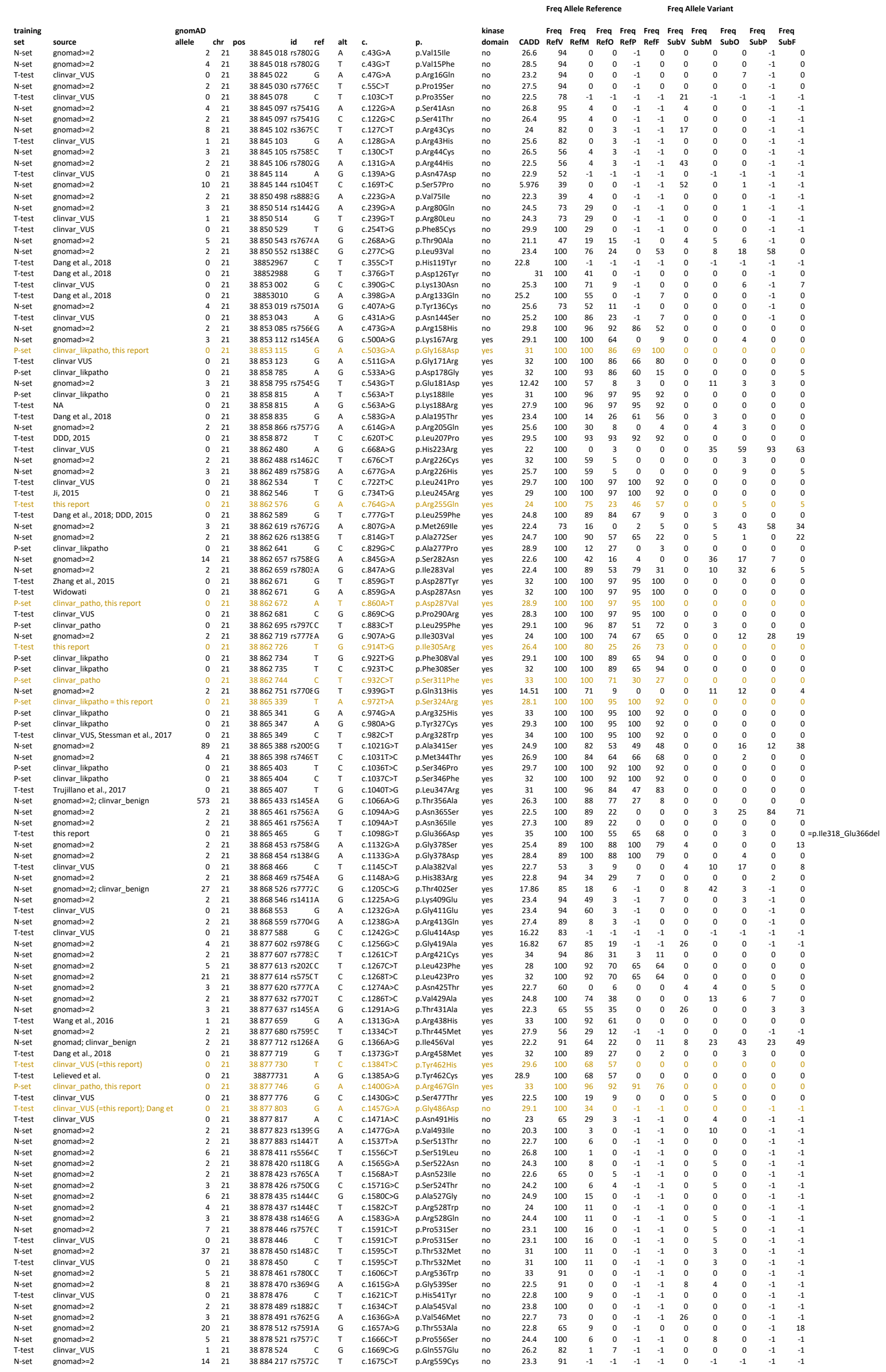




\begin{tabular}{|c|c|c|c|c|c|c|c|c|c|c|c|c|c|c|c|c|c|c|c|}
\hline N-set & gnomad $>=2$ & 32 & 21 & 38884218 rs $1441 \mathrm{G}$ & A & $c .1676 \mathrm{G}>\mathrm{A}$ & p.Arg559His & no & 22.7 & 91 & -1 & -1 & -1 & -1 & 0 & -1 & -1 & -1 & -1 \\
\hline N-set & gnomad $>=2$ & 2 & 21 & $38884227 \mathrm{rs} 7457 \mathrm{~T}$ & A & c.1685T>A & p.Phe562Tyr & no & 16.75 & 53 & -1 & -1 & -1 & -1 & 40 & -1 & -1 & -1 & -1 \\
\hline N-set & gnomad $>=2$ & 4 & 21 & 38884253 rs7797A & G & c. $1711 \mathrm{~A}>\mathrm{G}$ & p.Thr571Ala & no & 18.84 & 44 & -1 & -1 & -1 & -1 & 0 & -1 & -1 & -1 & -1 \\
\hline N-set & gnomad $>=2$ & 10 & 21 & $38884254 \mathrm{rs} 746 \in \mathrm{C}$ & G & $c .1712 C>G$ & p.Thr571Ser & no & 15.02 & 44 & -1 & -1 & -1 & -1 & 13 & -1 & -1 & -1 & -1 \\
\hline N-set & gnomad $>=2$ & 4 & 21 & $38884262 \mathrm{rs} 7682 \mathrm{C}$ & G & $c .1720 c>G$ & p.Pro574Ala & no & 24.7 & 80 & -1 & -1 & -1 & -1 & 0 & -1 & -1 & -1 & -1 \\
\hline $\mathrm{N}$-set & gnomad $>=2$ & 4 & 21 & 38884271 rs7713 G & c & $c .1729 G>C$ & p.Val577Leu & no & 24.6 & 89 & -1 & -1 & -1 & -1 & 0 & -1 & -1 & -1 & -1 \\
\hline $\mathrm{N}$-set & gnomad $>=2$ & 8 & 21 & 38884274 rs $774 C$ A & G & $c .1732 \mathrm{~A}>\mathrm{G}$ & p.Thr578Ala & no & 21.6 & 89 & -1 & -1 & -1 & -1 & 0 & -1 & -1 & -1 & -1 \\
\hline T-test & clinvar_vus & 0 & 21 & 38884281 & G & $c .1739 A>G$ & p.Glu580Gly & no & 26.7 & 89 & -1 & -1 & -1 & -1 & 0 & -1 & -1 & -1 & -1 \\
\hline N-set & gnomad $>=2$ & 2 & 21 & 38884286 rs $1293 \mathrm{C}$ & $\mathrm{T}$ & c. $1744 C>T$ & p. His582Tyr & no & 24.8 & 89 & -1 & -1 & -1 & -1 & 0 & -1 & -1 & -1 & -1 \\
\hline N-set & gnomad $>=2$ & 2 & 21 & 38884287 rs759c A & c & $c .1745 \mathrm{~A}>\mathrm{C}$ & p. His582Pro & no & 24.9 & 89 & -1 & -1 & -1 & -1 & 0 & -1 & -1 & -1 & -1 \\
\hline N-set & gnomad $>=2$ & 3 & 21 & 38884289 rs767\& C & A & $c .1747 C>A$ & p.Pro583Thr & no & 27.2 & 89 & -1 & -1 & -1 & -1 & 0 & -1 & -1 & -1 & -1 \\
\hline N-set & gnomad $>=2$ & 3 & 21 & $38884301 \mathrm{rs} 3681 \mathrm{~A}$ & G & $c .1759 A>G$ & p.Thr587Ala & no & 26.5 & 89 & -1 & -1 & -1 & -1 & 0 & -1 & -1 & -1 & -1 \\
\hline N-set & gnomad $>=2$ & 44 & 21 & 38884304 rs149c A & $c$ & c. $1762 A>C$ & p.Thr588Pro & no & 23.4 & 85 & -1 & -1 & -1 & -1 & 0 & -1 & -1 & -1 & -1 \\
\hline P-set & clinvar_likpatho & 0 & 21 & 38884305 & A & c. $1763 C>A$ & p. Thr588Asn & no & 22.8 & 85 & -1 & -1 & -1 & -1 & 0 & -1 & -1 & -1 & $=$ \\
\hline N-set & gnomad $>=2$ & 8 & 21 & 38884331 rs200\& G & A & $c .1789 \mathrm{G}>\mathrm{A}$ & p.Ala597Thr & no & 22.1 & 84 & -1 & -1 & -1 & -1 & 0 & -1 & -1 & -1 & -1 \\
\hline $\mathrm{N}$-set & gnomad $>=2$ & 3 & 21 & 38884347 rs $7583 \mathrm{~A}$ & $\mathrm{~T}$ & $c .1805 \mathrm{~A}>\mathrm{T}$ & p.His602Leu & no & 22.8 & 51 & -1 & -1 & -1 & -1 & 0 & -1 & -1 & -1 & -1 \\
\hline N-set & gnomad $>=2$ & 2 & 21 & 38884352 rs $146 \varepsilon \mathrm{A}$ & G & $c .1810 A>G$ & p.Asn604Asp & no & 22.7 & 61 & -1 & -1 & -1 & -1 & 0 & -1 & -1 & -1 & -1 \\
\hline N-set & gnomad $>=2$ & 2 & 21 & $38884359 \mathrm{rs} 3675 \mathrm{C}$ & A & $c .1817 C>A$ & p.Ser606Tyr & no & 24.2 & 72 & -1 & -1 & -1 & -1 & 0 & -1 & -1 & -1 & -1 \\
\hline N-set & gnomad $>=2$ & 4 & 21 & $38884388 \mathrm{rs} 760 \mathrm{C} C$ & G & $c .1846 C>6$ & p. His616Asp & no & 26.6 & 88 & -1 & -1 & -1 & -1 & 0 & -1 & -1 & -1 & -1 \\
\hline N-set & gnomad $>2$ & 2 & 21 & $38884398 \mathrm{rs} 754 \mathrm{CA}$ & G & $c .1856 \mathrm{~A}>\mathrm{G}$ & p.His619Arg & no & 23.2 & 88 & -1 & -1 & -1 & -1 & 0 & -1 & -1 & -1 & -1 \\
\hline $\mathrm{N}$-set & gnomad $>2$ & 3 & 21 & 38884412 rs7504T & G & c.1870T>G & p.Leu624Val & no & 22.3 & 62 & -1 & -1 & -1 & -1 & 0 & -1 & -1 & -1 & -1 \\
\hline N-set & gnomad $>=2$ & 8 & 21 & 38884421 rs $7583 \mathrm{C}$ & $\mathrm{T}$ & c.1879C>T & p.Arg627Trp & no & 32 & 80 & -1 & -1 & -1 & -1 & 0 & -1 & -1 & -1 & -1 \\
\hline N-set & gnomad $>=2$ & 5 & 21 & 38884422 rs7614G & A & c. $1880 \mathrm{G}>\mathrm{A}$ & p.Arg627GIn & no & 22.5 & 80 & -1 & -1 & -1 & -1 & 0 & -1 & -1 & -1 & -1 \\
\hline N-set & gnomad $>=2$ & 9 & 21 & 38884440 rs $147 \in A$ & $\mathrm{~T}$ & c.1898A>T & p. Tyr633Phe & no & 24.7 & 85 & -1 & -1 & -1 & -1 & 0 & -1 & -1 & -1 & -1 \\
\hline N-set & gnomad $>=2$ & 12 & 21 & $38884443 \mathrm{rs} 370 \mathrm{CA}$ & G & $c .1901 A>G$ & p.Asn634Ser & no & 22.6 & 77 & -1 & -1 & -1 & -1 & 8 & -1 & -1 & -1 & -1 \\
\hline $\mathrm{N}$-set & gnomad $>=2$ & 6 & 21 & 38884452 rs $1421 \mathrm{C}$ & $\mathrm{T}$ & c. $1910 \subset>T$ & p. Thr637Met & no & 23.4 & 81 & -1 & -1 & -1 & -1 & 0 & -1 & -1 & -1 & -1 \\
\hline N-set & gnomad $>2$ & 6 & 21 & 38884455 rs $775 c \mathrm{~A}$ & G & c. $1913 \mathrm{~A}>\mathrm{G}$ & p.Asn638Ser & no & 21.5 & 72 & -1 & -1 & -1 & -1 & 0 & -1 & -1 & -1 & -1 \\
\hline N-set & gnomad $>2$ & 4 & 21 & 38884484 rs $2014 \mathrm{G}$ & A & c. $1942 \mathrm{G}>\mathrm{A}$ & p.Val648lle & no & 26.8 & 94 & -1 & -1 & -1 & -1 & 0 & -1 & -1 & -1 & -1 \\
\hline N-set & gnomad $>=2$ & 2 & 21 & 38884505 rs7654A & G & $c .1963 A>G$ & p.Met655Val & no & 22.5 & 94 & -1 & -1 & -1 & -1 & 0 & -1 & -1 & -1 & 1 \\
\hline N-set & gnomad $>=2$ & 4 & 21 & 38884527 rs $7515 \mathrm{C}$ & $\mathrm{T}$ & c. $1985 \mathrm{C}>\mathrm{T}$ & p. Thr662Met & no & 24.9 & 51 & -1 & -1 & -1 & -1 & 0 & -1 & -1 & -1 & -1 \\
\hline N-set & gnomad $>=2$ & 2 & 21 & 38884556 rs7487A & G & $c .2014 A>G$ & p.Thr672Ala & no & 24.4 & 94 & -1 & -1 & -1 & -1 & 0 & -1 & -1 & -1 & -1 \\
\hline N-set & gnomad $>=2$ & 9 & 21 & 38884567 rs7567A & c & $c .2025 \mathrm{~A}>\mathrm{C}$ & p. Gln $675 \mathrm{His}$ & no & 22.3 & 60 & -1 & -1 & -1 & -1 & 34 & -1 & -1 & -1 & -1 \\
\hline $\mathrm{N}$-set & gnomad $>=2$ & 5 & 21 & 38884572 rs $543 \varepsilon \mathrm{A}$ & G & $c .2030 \mathrm{~A}>\mathrm{G}$ & p.Asn677Ser & no & 23.2 & 94 & -1 & -1 & -1 & -1 & 0 & -1 & -1 & -1 & -1 \\
\hline $\mathrm{N}$-set & gnomad $>=2$; clinvar benign & 388 & 21 & 38884577 rs5572G & c & $c .2035 \mathrm{G}>\mathrm{C}$ & p.Ala679Pro & no & 25.2 & 94 & -1 & -1 & -1 & -1 & 0 & -1 & -1 & -1 & -1 \\
\hline N-set & gnomad $>=2$; clinvar_benign & 8 & 21 & 38884589 rs2015C & $\mathrm{T}$ & $c .2047 C>T$ & p.Arg683Cys & no & 24.3 & 94 & -1 & -1 & 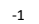 & $=$ & 0 & -1 & 1 & -1 & -1 \\
\hline N-set & gnomad $>=2$ & 57 & 21 & 38884640 rs5405 G & A & $c .2098 \mathrm{G}>\mathrm{A}$ & p.Val700Ile & no & 22.5 & 38 & -1 & -1 & -1 & -1 & 0 & -1 & -1 & -1 & -1 \\
\hline N-set & gnomad $>=2$ & 23 & 21 & 38884652 rs2005G & $\hat{A}$ & $c .2110 \mathrm{~A}>\mathrm{A}$ & p.Val704lle & no & 24.5 & 55 & -1 & -1 & -1 & -1 & 0 & -1 & -1 & -1 & -1 \\
\hline N-set & gnomad $>=2$ & 5 & 21 & $38884653 \mathrm{rs} 7637 \mathrm{~T}$ & c & $c .2111 T>C$ & p.Val704Ala & no & 22.4 & 55 & -1 & -1 & -1 & -1 & 38 & -1 & -1 & -1 & -1 \\
\hline N-set & gnomad $>=2$ & 9 & 21 & 38884662 rs $1513 \mathrm{~A}$ & G & $c .2120 A>G$ & p.Asn707Ser & no & 24.1 & 94 & -1 & -1 & -1 & -1 & 0 & -1 & -1 & -1 & -1 \\
\hline N-set & gnomad $>=2$ & 2 & 21 & 38884667 rs $781 \in \mathrm{C}$ & $\mathrm{T}$ & $c .2125 \mathrm{C}>\mathrm{T}$ & p.Arg709Cys & no & 33 & 94 & -1 & -1 & -1 & -1 & 0 & -1 & -1 & -1 & -1 \\
\hline N-set & gnomad $>=2$ & 4 & 21 & $38884668 \mathrm{rs} 3747 \mathrm{G}$ & A & $c .2126 \mathrm{G}>\mathrm{A}$ & p.Arg709His & no & 23.7 & 94 & -1 & -1 & -1 & -1 & 0 & -1 & -1 & -1 & -1 \\
\hline N-set & gnomad $>=2$ & 6 & 21 & 38884682 rs554€A & G & $c .2140 A>G$ & p.lle714Val & no & 22.2 & 55 & -1 & -1 & 2 & -1 & 8 & -1 & 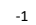 & -1 & -1 \\
\hline N-set & gnomad $>=2$ & 3 & 21 & 38884697 rs7744A & G & $c .2155 \mathrm{~A}>\mathrm{G}$ & o.Thr719Ala & no & 17.54 & 68 & -1 & -1 & 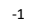 & -1 & 21 & -1 & -1 & -1 & 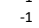 \\
\hline N-set & gnomad $>=2$ & 23 & 21 & 38884713 rs $1434 \mathrm{C}$ & G & $c .2171 C>G$ & p.Ala724Gly & no & 24.6 & 51 & -1 & -1 & -1 & -1 & 0 & -1 & -1 & -1 & -1 \\
\hline $\mathrm{N}$-set & gnomad $>=2$ & 2 & 21 & 38884715 rs $1401 \mathrm{~A}$ & c & $c .2173 \mathrm{~A}>\mathrm{C}$ & p.Asn725His & no & 26.5 & 90 & -1 & -1 & -1 & -1 & 0 & -1 & -1 & -1 & -1 \\
\hline T-test & clinvar_VUS & 1 & 21 & 38884719 & G & $c .2177 c>6$ & p.Thr726Arg & no & 24.4 & 94 & -1 & -1 & -1 & -1 & 0 & -1 & -1 & -1 & -1 \\
\hline N-set & gnomad $>=2$ & 12 & 21 & 38884722 rs7605 G & $\mathrm{T}$ & $c .2180 G>T$ & p.Gly727Val & no & 24.6 & 85 & -1 & -1 & -1 & -1 & 8 & -1 & -1 & -1 & -1 \\
\hline $\mathrm{N}$-set & gnomad $>=2$; clinvar_benign & 10 & 21 & 38884757 rs201c A & G & $c .2215 \mathrm{~A}>\mathrm{G}$ & p.Met739Val & no & 21.5 & 94 & -1 & -1 & -1 & -1 & 0 & -1 & -1 & -1 & -1 \\
\hline N-set & gnomad $>=2$ & 23 & 21 & 38884757 rs 201c A & $\mathrm{T}$ & c.2215A>T & p.Met739Leu & no & 21.3 & 94 & -1 & -1 & -1 & -1 & 0 & t & 2 & -1 & $=$ \\
\hline $\mathrm{N}$-set & & 3 & 21 & 38884767 rs749ع G & A & $c .22$ & Slu & no & 26 & 94 & -1 & -1 & -1 & -1 & 0 & -1 & -1 & -1 & -1 \\
\hline T-test & clit & 0 & 21 & 38884785 & T & & & no & 28 & 94 & 25 & 0 & -1 & -1 & 0 & 0 & 0 & -1 & -1 \\
\hline N-set & clinvar_benign & 0 & 21 & 38884788 rs $7532 \mathrm{C}$ & $\mathrm{T}$ & $c .2246 C>T$ & p.Pro749Leu & no & 29.3 & 94 & 25 & 0 & -1 & -1 & 0 & 0 & 0 & -1 & -1 \\
\hline N-set & gnomad $>=2$ & 22 & 21 & $38884793 \mathrm{rs} 147 \mathrm{CA}$ & $\mathrm{T}$ & $c .2251 \mathrm{~A}>\mathrm{T}$ & p.Th7751Ser & no & 22.7 & 77 & 0 & 0 & -1 & -1 & 0 & 0 & 0 & -1 & -1 \\
\hline N-set & gnomad $>=2$ & 2 & 21 & $38884830 \mathrm{rs} 7711 \mathrm{C}$ & G & $c .2288 C>G$ & p.Ser763Trp & no & 32 & 94 & -1 & -1 & -1 & -1 & 0 & -1 & -1 & -1 & -1 \\
\hline $\mathrm{N}$-set & gnomad $>=2$ & 4 & 21 & 38884830 rs7711C & $\mathrm{T}$ & $c .2288 \mathrm{C}>\mathrm{T}$ & p.Ser763Leu & no & 29.7 & 94 & -1 & -1 & -1 & -1 & 0 & -1 & -1 & -1 & -1 \\
\hline
\end{tabular}



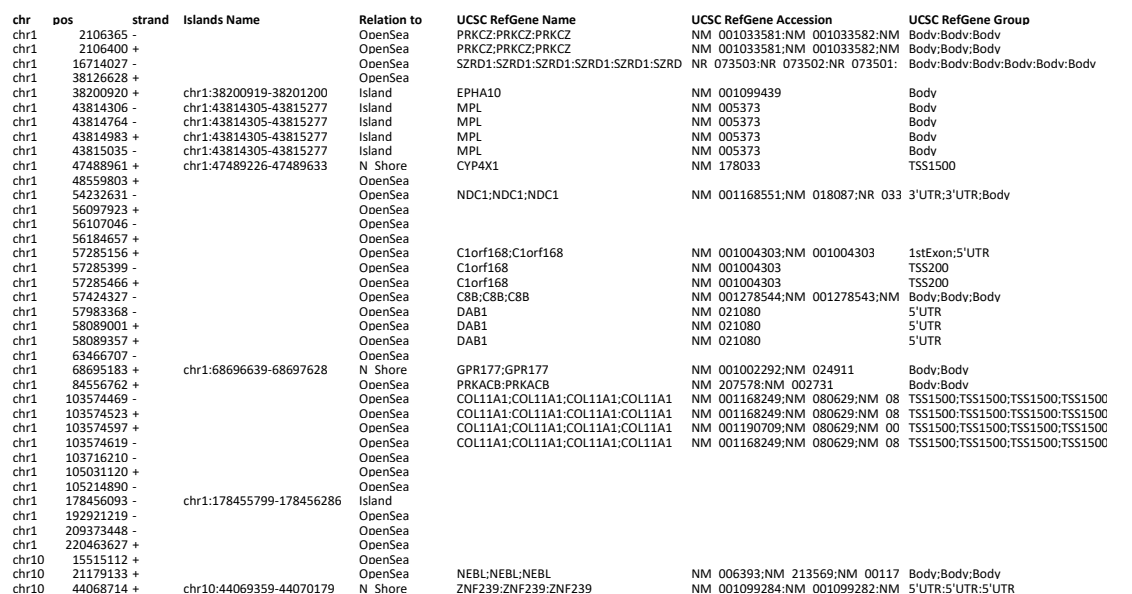

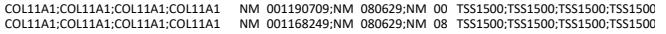

chr10:44069359-4407017

N Shore
Opensea
Opensea

chr10:72647738-72648317

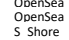

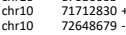

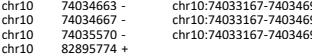

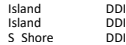

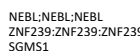

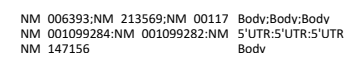$$
\text { che }
$$

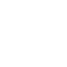

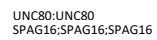

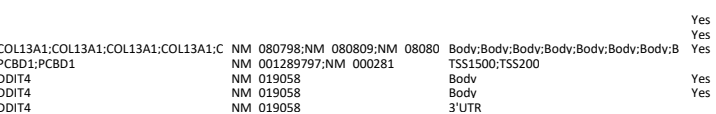

GRID1

HPSE2:HPSE2:HPSE2:HPS

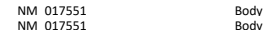

Dock1:Dock1
DOCK1:FAM196A

$\begin{array}{ll}\text { NM 001290223:NM 001380 } & \begin{array}{l}\text { Bodv:Bodv } \\ \text { Bodv:Bodv }\end{array} \\ \text { NM 001380:NM 001039762 } & \end{array}$

TSPAN4;TSPAN4;TSPAN4;TSPAN4;TSPAN NM 001025239:NM 001025237:NM 5'UTR:Bodv:Bodv:Bodv:Bodv:Bodv:Bodv $\begin{aligned} & \text { Yes } \\ & \text { Yes }\end{aligned}$

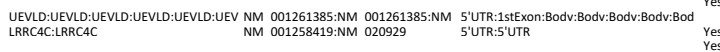

SHANK2

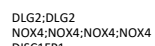

DISCIPP1
CRTAM:CRTAM

NM 01230

Bodv

PTMS
PTMS
PTMS

STrSIA1

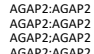

AGAPZ:AGAP2
AGPR:AGAP2

CAPS2:CAPS2:CAPS2:CAPS2
SYY15STI1:STY1
ACSS3

ACSS3
Loc101928

LNC01188
DTX1
GLT1D1

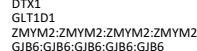

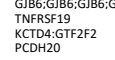

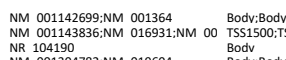

GRPPA-ASL:GRPP1-

NM 001304

NM 002824

NM 003034

Bodv
Bodv
Bodv

NM 001122772:NM 014770 Bodv

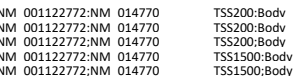

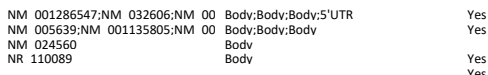

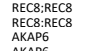

Uncoos21
CᄂMN

KIAA1370
FAMM214A:FAM214A:FAM214A
FAM214A

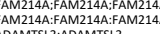

CETP:CEP
CEPPCETP

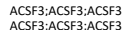

ATPAF2

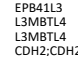

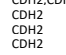

CCDC178:CDDC178:CCDC178

$\underset{\substack{\text { Fangac } \\ \text { fangac }}}{\text { and }}$

UnR:VRarivari

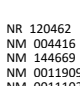

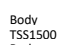

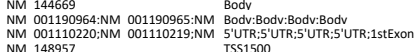

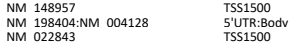

NR 120385:NN 046541:NM 00122867 Bodv:Bodv:Bodv:Bdy:Bodv
Body
NM 007368

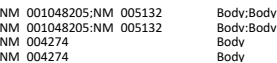

CTNNA2:CTNNA2;CTNNA2;CTNNA2 NM 001164883:NM 004389:NM 00 5'UTR:5'UTR:5'UTR:Bodv

SLCSA7

NM 021815

TSS1500 


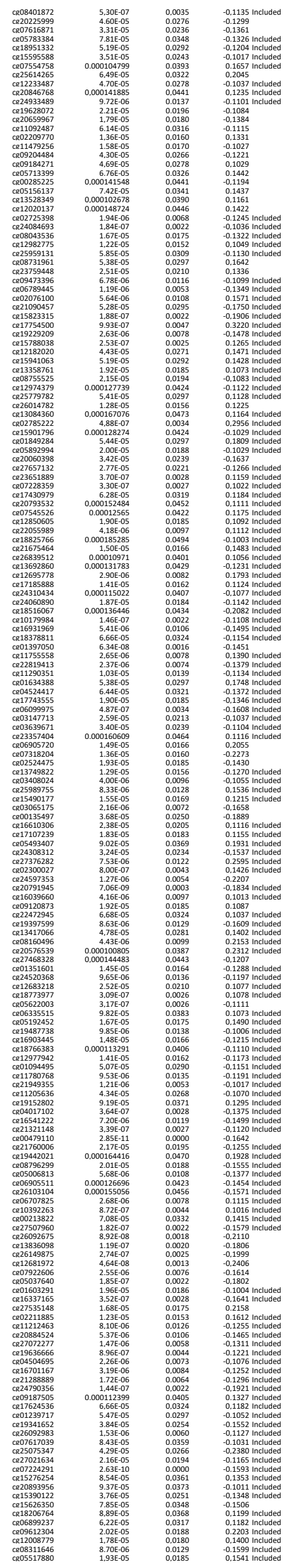
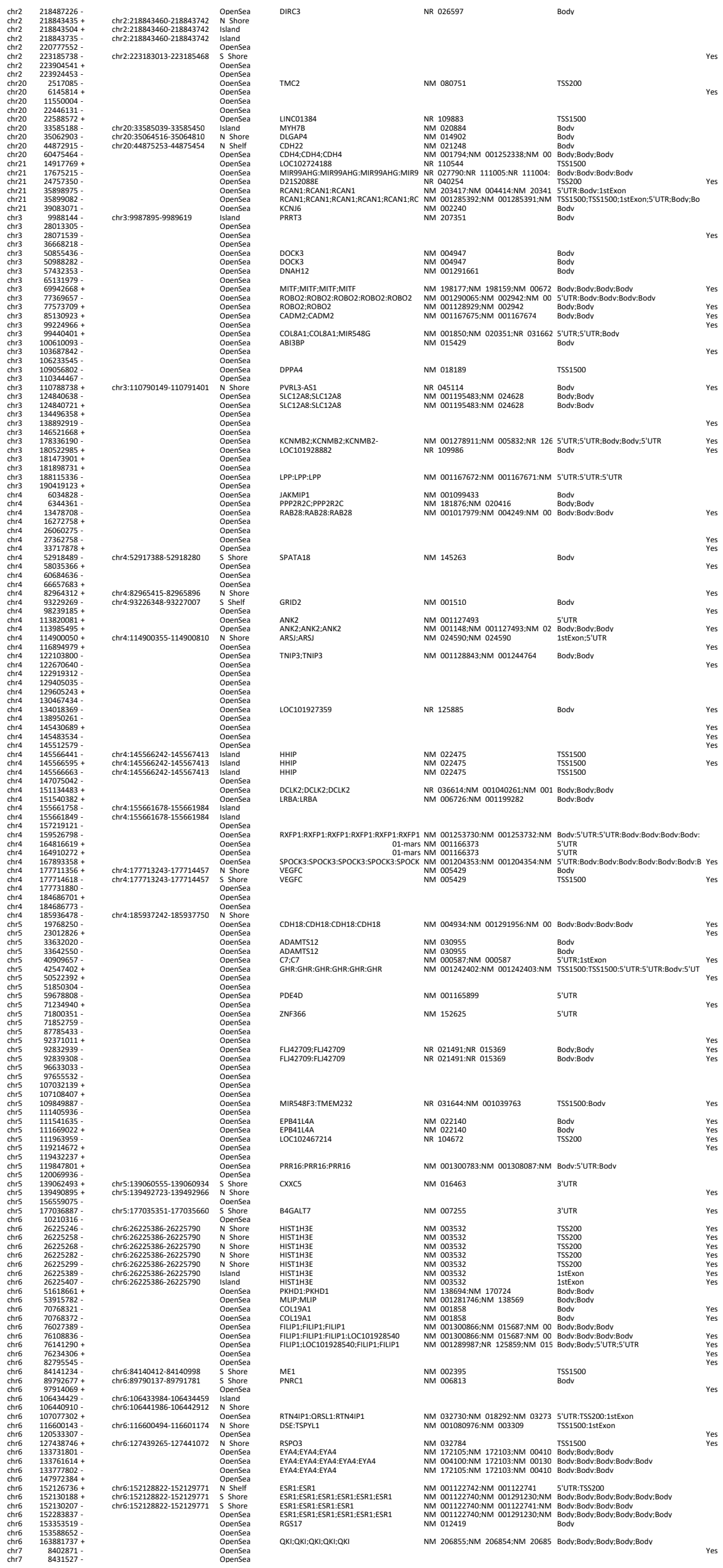
THSD7A

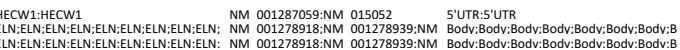

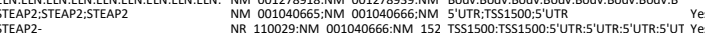

CDK14:CDK14:CDK14:CDK14
CALCR:CALCR:CALCR

$\underset{\mathrm{DGKK}}{\mathrm{UBE3C}}$

PXNL

STMN2:STMN2

CNGB3

LoC101927066

SAMD12:SAMD12:SAMD12
AMD12:SAMD12

12:SAMD12

\section{FAM84B
FAM84B
FAM84B}

MLLT3:MLLT3
LNCO1239:LINC01239

CDC14:CDC14B
RGS3:RGS3:RS3
RGS3:RGS3:RGS3:RGS3:RGS3

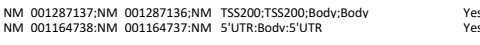

$\begin{array}{ll}\text { NM } 004717 & \begin{array}{l}\text { Bodv } \\ \text { MM } 014671\end{array} \\ \text { Body }\end{array}$

NM 144651

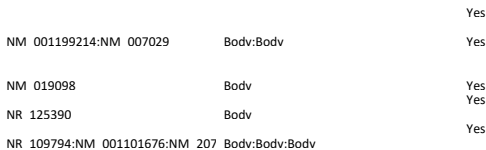

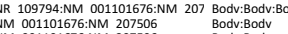

$\begin{array}{ll}\text { NM 174911 } & \text { Bodv } \\ \text { NM 17911 } & \text { Bodv } \\ \text { NM 17911 } & \text { Bodv } \\ \text { NM 001286691:NM 004529 } & \text { Bodv }\end{array}$

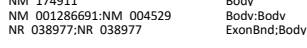

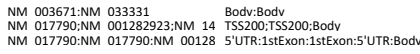

NM $033427 \quad$ Bodv 


\begin{tabular}{|c|c|c|c|c|}
\hline Sample_Group & Cohort \# & DYRK1A variant & SVM & SVM score \\
\hline test & Ind \#25 & p.Gly168Asp & positive & 0,7430 \\
\hline test & Ind \#26 & p.Arg255Gln & negative & 0,0709 \\
\hline test & Ind \#27 & p.Asp287Val & positive & 0,8478 \\
\hline test & Ind \#28 & p.lle305Arg & positive & 0,9347 \\
\hline test & Ruaud_\#2 & p.Ser311Phe & positive & 0,8190 \\
\hline test & Ind \#29 & p.Ser324Arg & positive & 0,9613 \\
\hline test & Ind \#31 & p.Tyr462His & negative & 0,0242 \\
\hline test & Ind \#32 & p.Arg467GIn & positive & 0,7874 \\
\hline test & Ind \#33 & p.Gly486Asp & GoF? & 0,0008 \\
\hline test & Bronicki_\#9 & p.Thr588Asn & negative & 0,0233 \\
\hline test & Ind \#18 & p.Ser660fs & positive & 0,9275 \\
\hline test_rep & Ind \#18_repl & p.Ser660fs_repl & positive & 0,9247 \\
\hline KMT2A & - & - & negative & 0,0849 \\
\hline KMT2A & - & - & negative & 0,0822 \\
\hline KMT2A & - & - & negative & 0,0590 \\
\hline KMT2A & - & - & negative & 0,0365 \\
\hline KMT2A & - & - & negative & 0,0216 \\
\hline KMT2A & - & - & negative & 0,0162 \\
\hline KMT2A & - & - & negative & 0,0137 \\
\hline KMT2A & - & - & negative & 0,0080 \\
\hline ARID1B & - & - & negative & 0,0254 \\
\hline ARID1B & - & - & negative & 0,0166 \\
\hline ARID1B & - & - & negative & 0,0104 \\
\hline ARID1B & - & - & negative & 0,0092 \\
\hline Control & - & - & negative & 0,1394 \\
\hline Control & - & - & negative & 0,1052 \\
\hline Control & - & - & negative & 0,0920 \\
\hline Control & - & - & negative & 0,0892 \\
\hline Control & - & - & negative & 0,0827 \\
\hline Control & - & - & negative & 0,0720 \\
\hline Control & - & - & negative & 0,0682 \\
\hline Control & - & - & negative & 0,0657 \\
\hline Control & - & - & negative & 0,0583 \\
\hline Control & - & - & negative & 0,0563 \\
\hline Control & - & - & negative & 0,0509 \\
\hline Control & - & - & negative & 0,0504 \\
\hline Control & - & - & negative & 0,0501 \\
\hline Control & - & - & negative & 0,0495 \\
\hline Control & - & - & negative & 0,0483 \\
\hline Control & - & - & negative & 0,0439 \\
\hline Control & - & - & negative & 0,0403 \\
\hline Control & - & - & negative & 0,0391 \\
\hline Control & - & - & negative & 0,0372 \\
\hline Control & - & - & negative & 0,0353 \\
\hline Control & - & - & negative & 0,0351 \\
\hline Control & - & - & negative & 0,0349 \\
\hline Control & - & - & negative & 0,0345 \\
\hline Control & - & - & negative & 0,0343 \\
\hline Control & - & - & negative & 0,0309 \\
\hline Control & - & - & negative & 0,0292 \\
\hline Control & - & - & negative & 0,0289 \\
\hline Control & - & - & negative & 0,0286 \\
\hline Control & - & - & negative & 0,0286 \\
\hline Control & - & - & negative & 0,0277 \\
\hline Control & - & - & negative & 0,0270 \\
\hline Control & - & - & negative & 0,0260 \\
\hline Control & - & - & negative & 0,0258 \\
\hline Control & - & - & negative & 0,0254 \\
\hline Control & - & - & negative & 0,0251 \\
\hline Control & - & - & negative & 0,0240 \\
\hline Control & - & - & negative & 0,0223 \\
\hline
\end{tabular}




\begin{tabular}{|c|c|c|c|c|}
\hline Control & - & - & negative & 0,0218 \\
\hline Control & - & - & negative & 0,0215 \\
\hline Control & - & - & negative & 0,0214 \\
\hline Control & - & - & negative & 0,0212 \\
\hline Control & - & - & negative & 0,0210 \\
\hline Control & - & - & negative & 0,0210 \\
\hline Control & - & - & negative & 0,0204 \\
\hline Control & - & - & negative & 0,0196 \\
\hline Control & - & - & negative & 0,0196 \\
\hline Control & - & - & negative & 0,0190 \\
\hline Control & - & - & negative & 0,0190 \\
\hline Control & - & - & negative & 0,0186 \\
\hline Control & - & - & negative & 0,0184 \\
\hline Control & - & - & negative & 0,0183 \\
\hline Control & - & - & negative & 0,0177 \\
\hline Control & - & - & negative & 0,0168 \\
\hline Control & - & - & negative & 0,0167 \\
\hline Control & - & - & negative & 0,0162 \\
\hline Control & - & - & negative & 0,0162 \\
\hline Control & - & - & negative & 0,0159 \\
\hline Control & - & - & negative & 0,0158 \\
\hline Control & - & - & negative & 0,0152 \\
\hline Control & - & - & negative & 0,0148 \\
\hline Control & - & - & negative & 0,0147 \\
\hline Control & - & - & negative & 0,0142 \\
\hline Control & - & - & negative & 0,0138 \\
\hline Control & - & - & negative & 0,0137 \\
\hline Control & - & - & negative & 0,0137 \\
\hline Control & - & - & negative & 0,0125 \\
\hline Control & - & - & negative & 0,0125 \\
\hline Control & - & - & negative & 0,0120 \\
\hline Control & - & - & negative & 0,0120 \\
\hline Control & - & - & negative & 0,0119 \\
\hline Control & - & - & negative & 0,0115 \\
\hline Control & - & - & negative & 0,0114 \\
\hline Control & - & - & negative & 0,0112 \\
\hline Control & - & - & negative & 0,0112 \\
\hline Control & - & - & negative & 0,0110 \\
\hline Control & - & - & negative & 0,0110 \\
\hline Control & - & - & negative & 0,0103 \\
\hline Control & - & - & negative & 0,0103 \\
\hline Control & - & - & negative & 0,0099 \\
\hline Control & - & - & negative & 0,0099 \\
\hline Control & - & - & negative & 0,0096 \\
\hline Control & - & - & negative & 0,0092 \\
\hline Control & - & - & negative & 0,0089 \\
\hline Control & - & - & negative & 0,0089 \\
\hline Control & - & - & negative & 0,0088 \\
\hline Control & - & - & negative & 0,0083 \\
\hline Control & - & - & negative & 0,0081 \\
\hline Control & - & - & negative & 0,0073 \\
\hline Control & - & - & negative & 0,0072 \\
\hline Control & - & - & negative & 0,0069 \\
\hline Control & - & - & negative & 0,0067 \\
\hline Control & - & - & negative & 0,0061 \\
\hline Control & - & - & negative & 0,0056 \\
\hline Control & - & - & negative & 0,0049 \\
\hline
\end{tabular}




\begin{tabular}{|c|c|c|c|c|c|c|c|c|c|c|c|c|c|c|}
\hline \multicolumn{3}{|c|}{ Variant } & \multicolumn{2}{|c|}{ In silico } & \multicolumn{4}{|c|}{ In vitro studies } & \multicolumn{4}{|c|}{ Individuals } & \multicolumn{2}{|c|}{ Final classification } \\
\hline Variant & 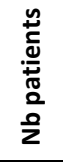 & 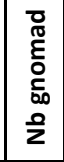 & 高 & 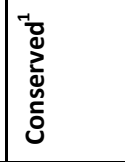 & 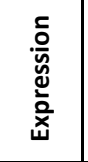 & $\begin{array}{l}0 \\
\stackrel{0}{*} \\
\dot{z}\end{array}$ & 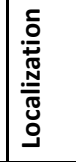 & $\begin{array}{l}\text { Results previously } \\
\text { reported }\end{array}$ & Report & $\left|\begin{array}{l}\text { Clinical features } \\
\left(\text { CS }_{\text {DYRK1A) }}\right.\end{array}\right|$ & 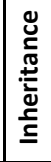 & DNAm profil & ACMG/AMP criteria & $\begin{array}{l}\text { Final } \\
\text { classification }\end{array}$ \\
\hline p.Ser660fs & 1 & 0 & & $\mathrm{NA}$ & NA & NA & Aggr. & $\mathrm{NA}$ & this report Ind \#18 & poorly $(7.5 / 20)$ & dn & positive & & Lik Patho \\
\hline p.Ala341Ser & 0 & 89 & 24,9 & midly & $100 \%$ & $100 \%$ & ns & NA & - & - & - & - & BS1, BS2, BS3, BP4 & Benign \\
\hline \multirow{2}{*}{ p.Gly168Asp } & \multirow{2}{*}{2} & \multirow{2}{*}{0} & \multirow{2}{*}{31} & \multirow{2}{*}{ highly } & \multirow{2}{*}{$<50 \%$} & \multirow{2}{*}{$0 \%$} & \multirow{2}{*}{ ns } & \multirow{2}{*}{ NA } & this report Ind \#25 & NA & $\mathrm{dn}$ & positive & \multirow{2}{*}{ PS2, PS3, PM2, PP3 } & \multirow{2}{*}{ Patho } \\
\hline & & & & & & & & & GeneDx (clinvar SCV00057310) & NA & NA & NA & & \\
\hline p.Arg255Gln & 1 & 0 & 24 & midly & $100 \%$ & $100 \%$ & ns & $\mathrm{NA}$ & this report Ind \#26 & poorly (5/15) & ? & negative & BS3, BP4 & Lik Benign \\
\hline \multirow{3}{*}{ p.Asp287Val } & \multirow{3}{*}{3} & \multirow{3}{*}{0} & \multirow{3}{*}{28,9} & \multirow{3}{*}{ highly } & \multirow{3}{*}{$<25 \%$} & \multirow{3}{*}{$0 \%$} & \multirow{3}{*}{ ns } & \multirow{3}{*}{$\begin{array}{l}\text { drastic decrease of autoP } \\
\text { and kinase activity (1) }\end{array}$} & DDD (Decipher 258963) & highly ${ }^{a}$ & dn & NA & \multirow{3}{*}{ PS2, PS3, PM2,PP3, PP4 } & \multirow{3}{*}{ Patho } \\
\hline & & & & & & & & & $\begin{array}{l}\text { this report Ind \#27 (Geneva Hospital, } \\
\text { scv000598121) }\end{array}$ & interm. (10/15) & dn & positive & & \\
\hline & & & & & & & & & Tubingen (clinvar SCV00144673) & intermediate $^{i}$ & NA & NA & & \\
\hline p.Ile305Arg & 1 & 0 & 26,9 & midly & $<25 \%$ & $0 \%$ & ns & NA & this report Ind \#28 & highly $(16 / 20)$ & dn & positive & PS2, PS3, PM2, PP4 & Patho \\
\hline p.Ser311Phe & 2 & 0 & 33 & moderate & $<50 \%$ & $0 \%$ & ns & drastic decrease of autoP & Ruaud_\#2 (clinvar SCV000586742) & interm. $(12,5 / 20)$ & dn & positive & PS2, PS3, PM2, PP3 & Patho \\
\hline & & & & & & & & and kinase activity $(1,2)$ & GeneDx (clinvar SCV000520979) & NA & NA & NA & & \\
\hline p.Ser324Arg & 1 & 0 & 28,1 & highly & $80 \%$ & $<25 \%$ & ns & NA & this report Ind \#29 (SCV000902439) & interm. $(10,5 / 20)$ & dn & positive & PS2, PS3, PM2, PP3 & Patho \\
\hline p.Glu366Asp* & 1 & 0 & $35 *$ & midly & $100 \%$ & $100 \%$ & ns & NA & this report Ind \#30 & highly (16/20) & dn & NA & $\begin{array}{l}\text { PS2, PS3, PM2, PP4, } \\
\text { PP3 }\end{array}$ & Patho * \\
\hline p.Tyr462His & 1 & 0 & 29,6 & midly & $100 \%$ & $100 \%$ & ns & NA & this report Ind \#31 & poorly $(6,5 / 20)$ & dn & negative & PS2, PM2, BS3, & Lik Benign \\
\hline & & & & & & & & & DDD (Decipher 270174) & poorly $^{b}$ & $\mathrm{dn}$ & NA & & \\
\hline & & & & & & & & drastic decrease of autop & Posey et al., 2016 (clinvar SCV000245477) & interm. ${ }^{c}$ & $\mathrm{dn}$ & NA & PS2, PS3, PM1, PM2, & \\
\hline p.Arg467Gln & 4 & 0 & 33 & highly & $<50 \%$ & $0 \%$ & ns & and kinase activity $(1,2)$ & GeneDx (clinvar SCV000534701) & NA & NA & NA & PP3 & Patho \\
\hline & & & & & & & & & this report Ind \#32 & interm. (10.5/20) & dn & positive & & \\
\hline & & & & & & & & & Dang et al., 2018 & NA & NA & NA & & \\
\hline p.Gly486Asp & 2 & 0 & 29,1 & midly & $100 \%$ & $100 \%$ & ns & no decrease of kinase acti & $\begin{array}{l}\text { this report Ind \#33 (Geneva Hospital, } \\
\text { scV000747759) }\end{array}$ & poorly $(2 / 15)^{d}$ & dn & GoF? & PS2, PM2, BS3 & VUS \\
\hline p.Thr588Asn & $1^{* *}$ & $0^{* * *}$ & 22,8 & midly & $100 \%$ & $100 \%$ & ns & no effect on autoP and $P$ & $\begin{array}{l}\text { Bronicki_\#9 (clinvar SCV000196065 = } \\
\text { SCV000965705)** }\end{array}$ & highly $(15.5 / 20)$ & dn & negative & $\begin{array}{l}\text { PS2, PM2, PP4, BS3, } \\
\text { BP4 }\end{array}$ & Lik Benign \\
\hline p.Arg158His & 1 & 2 & 29,8 & highly & $100 \%$ & $100 \%$ & NA & $\mathrm{NA}$ & DDD (Deciper_LikBenign) & $N A^{e}$ & $\mathrm{dn}$ & NA & PS2, PP3, BS2, BS3 & Benign \\
\hline Ala277Pro & 2 & 0 & 28.9 & midly & $50 \%$ & $0 \%$ & INA & drastic decrease of autop & DDD (Deciper_Patho) & interm. ${ }^{f}$ & $\mathrm{dn}$ & NA & PS2 PS3, PM2 & Patho \\
\hline P.Ald & 2 & 0 & | & Jiming & $50 \%$ & $0 \%$ & IVA & jarastic aecrease or autor & GeneDx (SCV000571206) & NA & NA & NA & |RSL, PSS, PIVIL & Jatno \\
\hline p.Gly171Arg & 1 & 0 & 32 & highly & $50 \%$ & $0 \%$ & NA & NA & Invitae (clinvar SCV000952112.2_LikelyPatho) & interm. ${ }^{\mathrm{g}}$ & dn & NA & PS2, PS3, PM2, PP3 & Patho \\
\hline p.Leu241Pro & 1 & 0 & 29,7 & highly & $\sim 50 \%$ & $0 \%$ & NA & NA & EGL diagnostic (clinvar SCV000703832.2_VUS) & NA & NA & NA & PS3, PM2, PP3 & Lik Patho \\
\hline p.Pro290Arg & 1 & 0 & 28,3 & highly & \begin{tabular}{|l|l|}
$<50 \%$ \\
\end{tabular} & $0 \%$ & NA & NA & Ambry (clinvar SCV000741841.1_VUS) & interm. $^{\mathrm{h}}$ & NA & NA & PS3, PM2, PP3 & Lik Patho \\
\hline
\end{tabular}

\section{ACCEPTED MANUSCRIPT - CLEAN COPY}




\begin{tabular}{|c|c|c|c|c|c|c|c|c|c|c|}
\hline \multicolumn{3}{|c|}{ Variant } & \multicolumn{2}{|c|}{ In silico } & \multirow{2}{*}{\begin{tabular}{|l} 
In vitro studies \\
Results previously reported
\end{tabular}} & \multicolumn{3}{|l|}{ Individuals } & \multicolumn{2}{|c|}{ Final classification } \\
\hline Variant & 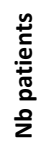 & 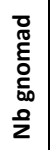 & 姹 & 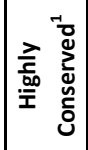 & & Report & \begin{tabular}{|l} 
Clinical \\
features \\
(CS DYRK $1 A))$
\end{tabular} & 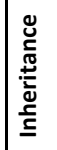 & ACMG/AMP criteria & $\begin{array}{l}\text { Final } \\
\text { classification }\end{array}$ \\
\hline His119Tyr & 1 & 0 & 22.8 & no & no effect on neurite outgrowth (Dang et al.) & Dang et al., 2018 & intermediate $^{\mathrm{a}}$ & $\mathrm{NA}$ & PM2, BS3 & Lik benign \\
\hline Lys188lle & 1 & 0 & 31 & yes & drastic decrease of autoP and kinase activity (Arranz) & Ji et al., 2015 (clinvar SCV000206791_LikPatho) & highly $(13 / 20)$ & $\mathrm{dn}$ & PS2,PS3, PM2, PP3, PP4 & Patho \\
\hline Ala195Thr & 1 & 0 & 23,4 & no & $\begin{array}{l}\text { Increase kinase activity (Arranz et al.)?, no effect on neurite outgrowth } \\
\text { (Dang et al.) }\end{array}$ & Dang et al., 2018 & poorly ${ }^{b}$ & NA & PM2, BS3, BP4 & Lik benign \\
\hline Leu207Pro & 1 & 0 & 29,5 & yes & drastic decrease of autoP and kinase activity (Arranz) & DDD (Decipher 259211_Patho) & intermediate $^{c}$ & $\mathrm{dn}$ & PS2,PS3, PM2, PP3 & Patho \\
\hline His223Arg & 1 & 0 & 22,0 & no & Increase kinase activity (Arranz) et al.? & GeneDx (clinvar SCV000620751_VUS) & $\mathrm{NA}$ & $\mathrm{NA}$ & PM2, BS3, BP4 & Lik benign \\
\hline \multirow{2}{*}{-eu245Arg } & \multirow{2}{*}{2} & \multirow{2}{*}{0} & \multirow{2}{*}{29} & \multirow{2}{*}{ yes } & \multirow{2}{*}{$\begin{array}{l}\text { drastic decrease of autoP and kinase activity (Widowati et al.,Arranz et } \\
\text { al.) }\end{array}$} & Ji et al., 2015 (clinvar SCV000206792_LikPatho) & highly (12/15) & $\mathrm{dn}$ & \multirow{2}{*}{ PS2,PS3, PM2, PP4, PP3 } & \multirow[b]{2}{*}{ Patho } \\
\hline & & & & & & Baylor college (clinvar SCV000807304_VUS) & intermediate $^{d}$ & $\mathrm{dn}$ & & \\
\hline Leu259Phe & 1 & 0 & 24,8 & no & $\begin{array}{l}\text { Increase kinase activity (Arranz et al.) ?, no effect on neurite outgrowth } \\
\text { (Dang et al.) }\end{array}$ & Dang et al., 2018 & poorlye & NA & PM2, BS3, BP4 & Lik benign \\
\hline Asp287Tyr & 1 & 0 & 32 & yes & drastic decrease of autoP and kinase activity (Arranz et al;) & Zhang et al., 2015 & intermediate $^{\mathrm{f}}$ & $\mathrm{dn}$ & PS2,PS3, PM2, PP3 & VUS \\
\hline \multirow{2}{*}{ Leu295Phe } & \multirow{2}{*}{2} & \multirow{2}{*}{0} & \multirow{2}{*}{29,1} & \multirow{2}{*}{ yes } & \multirow{2}{*}{ slight decrease kinase activity (Widowati et al.,Arranz et al.) } & Ji et al., 2015 = SCV000206793 (UCLA_LikPatho) & poorly (5.5/15) & $\mathrm{dn}$ & \multirow{2}{*}{ PS2,PS3, PM2, PP3 } & \multirow{2}{*}{ VUS } \\
\hline & & & & & & GeneDx Clinvar SCV000321572_Patho) & NA & NA & & \\
\hline Phe308Val & 1 & 0 & 29,1 & yes & $\begin{array}{l}\text { drastic decrease of autoP and kinase activity (Widowati et al., Arranz et } \\
\text { al.) }\end{array}$ & Chicago Hospital (clinvar SCV000247240_LikPatho) & NA & NA & PS3, PM2, PM2, PP3 & Lik patho \\
\hline Gln313His & 0 & 2 & 14,5 & no & slight decrease kinase activity (Widowati et al.,Arranz et al.) & - & - & - & - & - \\
\hline Arg325His & 1 & 0 & 33 & yes & drastic decrease of autoP and kinase activity (Arranz et al.) & GeneDx (clinvar SCV000574147_LikPatho) & $\mathrm{NA}$ & $\mathrm{dn}$ & PS2,PS3, PM2, PP3 & Lik patho \\
\hline Tyr327Cys & 1 & 0 & 29,3 & yes & drastic decrease of autoP and kinase activity (Arranz et al.) & BC Hospital (clinvar SCV000599256_LikPatho) & NA & $\mathrm{dn}$ & PS2,PS3, PM2, PP3 & Lik patho \\
\hline Arg328Trp & 1 & 0 & 34 & yes & drastic decrease of autoP and kinase activity (Arranz et al.) & GeneDx (clinvar SCV000492371_VUS) & $\mathrm{NA}$ & $\mathrm{NA}$ & PS3, PM2, PP3 & Lik patho \\
\hline Ser346Phe & 1 & 0 & 32 & yes & drastic decrease of autoP and kinase activity (Arranz et al.) & LMM Cambridge (Clinvar SCV000712522_LikPatho) & intermediate $^{\mathrm{g}}$ & $\mathrm{dn}$ & PS2,PS3, PM2, PP3 & Patho \\
\hline \multirow{2}{*}{ Ser346Pro } & \multirow{2}{*}{2} & \multirow{2}{*}{0} & \multirow{2}{*}{29,7} & \multirow{2}{*}{ yes } & \multirow{2}{*}{$\begin{array}{l}\text { drastic decrease of autoP and kinase activity (Widowati et al.,Arranz et } \\
\text { al.) }\end{array}$} & Bronicki et al., 2015 (Clinvar SCV000196060_LikPatho) & highly $^{\mathrm{h}}$ & $\mathrm{dn}$ & PS2,PS3, PM2, PP3, PP4 & Patho \\
\hline & & & & & & DDD (decipher 260956_Patho) & intermediate $^{i}$ & $\mathrm{dn}$ & PS2,PS3, PM2, PP3 & Patho \\
\hline Leu347Arg & 1 & 0 & 31 & yes & drastic decrease of autoP and kinase activity (Arranz et al.) & Trujillano et al., 2017 & highly $^{j}$ & $\mathrm{dn}$ & PS2,PS3, PM2, PP3, PP4 & Patho \\
\hline Arg438His & 1 & 1 & 33 & no & sklight decrease in kinase activity (Arranz et al..)? & Wang et al., 2016 & NA & $\mathrm{dn}$ & PS2, BS1, BS3 & Lik benign \\
\hline Arg458Met & 1 & 0 & 32 & no & Increase kinase activity (Arranz et al.)? & Dang et al., 2018 & $\mathrm{NA}$ & NA & $\mathrm{BS} 3, \mathrm{PM} 2$ & VUS \\
\hline Arg528Trp & 1 & 4 & 24 & no & Increase kinase activity (Arranz et al.)? & Chicago Hospital (clinvar SCV000594478_VUS) & NA & NA & BS1, BS3, BP4 & Benign \\
\hline
\end{tabular}

\section{ACCEPTED MANUSCRIPT - CLEAN COPY}




\begin{tabular}{|l|l|l|l|l|}
\hline Name & Identifier & Classification & Inheritance & Clinical features \\
\hline c.1726C>T p.Gln576* & Okamato et al. 2014 & $\begin{array}{l}\text { considered as disease } \\
\text { causing }\end{array}$ & NA & poorly ${ }^{\text {a }}$ \\
\hline c.2040C>A p.Tyr680* & ClinVar (Genedx, SCV000779549.2) & Likely pathogenic & NA & poorly to intermediate ${ }^{\text {b }}$ \\
\hline c.2213_2218delinsAGAG p.Thr738fs & ClinVar (Genedx, SCV000570988.4) & Likely pathogenic & de novo & intermediate $^{c}$ \\
\hline
\end{tabular}

